\title{
SOURCE-CHANNEL CODEC FOR A WCDMA BASED MULTIMEDIA SYSTEM
}

by

Boris Backovic, B.Eng.

Toronto, September $22^{\text {st }}, 2005$

\author{
A Project Report \\ presented to Ryerson University \\ in partial fulfillment of the \\ requirements for the degree \\ Master of Engineering \\ in the Program of \\ Electrical Engineering
}

Toronto, Ontario, Canada, 2005

(C) Boris Backovic 2005 


\title{
UMI Number: EC53005
}

\section{All rights reserved \\ INFORMATION TO USERS}

The quality of this reproduction is dependent upon the quality of the copy submitted. Broken or indistinct print, colored or poor quality illustrations and photographs, print bleed-through, substandard margins, and improper alignment can adversely affect reproduction.

In the unlikely event that the author did not send a complete manuscript and there are missing pages, these will be noted. Also, if unauthorized copyright material had to be removed, a note will indicate the deletion.

\section{$\mathrm{UMI}^{\circ}$}

UMI Microform EC53005

Copyright 2008 by ProQuest LLC

All rights reserved. This microform edition is protected against unauthorized copying under Title 17, United States Code.

\author{
ProQuest LLC \\ 789 East Eisenhower Parkway \\ P.O. Box 1346 \\ Ann Arbor, MI 48106-1346
}





\section{The Journey of Success}

When choosing the path to follow, I selected the road heading west. It began in the Forest of Childhood, and ceased at the City of Success.

My bag was packed full of knowledge, but also some fears and some weights.

My most precious cargo was a vision of entering the City's bright gates.

I reached an impassable river, and feared that my dream had been lost. But I found a sharp rock, cut down a tree, and created a bridge, which I crossed.

It started to rain, and I was so cold, I shivered and started to doubt. But I made an umbrella out of some leaves and kept all the cold water out.

The journey took longer that I had planned; I had no food left in my dish. Rather than starve before reaching my dream, I taught myself how to fish.

I grew awfully tired as I walked on and on, and I thought of the weights in my pack. I tossed them aside, and I sped up again. Fear was all that was holding me back.

I could see the City of Success, just beyond a small grove of trees. At last, I thought, I have reached my goal! The whole world will envy me!

I arrived at the city, but the gate was locked. The man at the door frowned and hissed, "You have wasted your time. I can't let you in. Your name is not on my list."

I cried and I screamed and I kicked and I shook; I felt that my life had just ceased. For the first time ever, I turned my head, and for once in my life faced the east.

I saw all the things I had done on my way, all the obstacles I'd overcome. I couldn't enter the City, but that didn't mean I hadn't won.

I had taught myself how to ford rivers, and how to stay dry in the rain. I had learned how to keep my heart open, even if sometimes it lets in some pain.

I learned, facing backwards, that life meant more than just survival. My success was in my journey, not in my arrival. 


\begin{abstract}
The project deals with the operation of a Source-Channel Codec for a WCDMA Based Multimedia System. The system is meant to transfer and receive both digitized speech and still image signals. It uses a part of the WCDMA technology to mix up the transmitted signals throughout the implementation of Direct Sequence Spread Spectrum and Chip Sequencing methodologies. The Walsh code algorithm is used to ensure the orthogonality among different Chip Sequences.
\end{abstract}

On the transmitter side the system first offers the formatting stage where both a speech and a still image signal are digitized. The following stage in the system exhibits a significant degree of data compression applying appropriate compression algorithms: Lempel-Ziv-Welch for the speech signal and Huffman Code Algorithm for the still image. These compression algorithms are implemented in the Source Encoder stage of the system. The system also provides basic FEC (Forward Error Correction) capabilities, using both Linear Block Code and Convolutional Code algorithms introduced in the Channel Encoder stage. The goal of these FEC algorithms is to detect and correct errors during the transmission of data due to the channel imperfections. At the WCDMA stage the two signals are added together forming an aggregated signal that is being transmitted through the channel.

On the receiver side a digital demodulator separates the aggregated signal to into two signals using the feature of the orthogonality of vectors. Then the Channel Decoder stage follows, where both signals, which have gotten corrupted during the transmission through the channel due to channel imperfections, are recovered. The imperfections in the channel are simulated by random noise that is added to the aggregated signal in the WCDMA stage of the system. The last stage in the system, the Source Decoder stage, deals with the conversion of the received signals from the digital to analog form and reconstruction of the signals in the sense that they can again be heard (speech) and seen (still image).

Each stage in the system is simulated using MATLAB programming language. The report is formed of three major parts; the theoretical part where the theory behind each stage in the system is explained, the example part where applicable numerical examples are provided and analyzed for better understanding of both the theory and the Matlab code, and the result part where the Matlab results for each stage are analyzed. 


\section{Table of Contents}

Abstract I

Chapter 1: INTRODUCTION 1

$\begin{array}{ll}\text { Chapter 2: FORMATTING } & 7\end{array}$

$\begin{array}{ll}2.1 \text { Speech Formatting } & 7\end{array}$

2.1.1 Sampling $\quad 8$

2.1.2 Quantizing 9

$\begin{array}{ll}2.1 .3 \text { Pulse Code Modulation (PCM) } & 13\end{array}$

2.1.4 Matlab Implementation of Formatting $\quad 14$

$\begin{array}{lr}2.2 \text { Image Formatting } & 19\end{array}$

$\begin{array}{ll}\text { Chapter 3: SOURCE CODEC } & 23\end{array}$

3.1 Huffman Coding Algorithm 23

3.1.1 Huffman Encoding 25

3.1.2 Huffman Decoding $\quad 28$

3.1.3 Matlab Implementation of Huffman Coding Algorithm 30

3.2 Lempel-Ziv-Welch Algorithm 32

3.2.1 Matlab Implementation of Lempel-Ziv-Welch Algorithm $\quad$ • 37

Chapter 4: CHANNEL CODEC 41

4.1 Channel Codes 41

4.1.1 Parity Check Codes 41

4.1.2 Linear Block Codes 43

4.1.3 Linear Block Code Encoding 45

4.1.4 Linear Block Code Decoding 46

4.1.5 Matlab Implementation of Linear Block Codes 53

4.2 Convolutional Codes $\quad 54$

4.2.1 Convolutional Code Encoding $\quad 56$

4.2.1.1 Impulse Response of the Convolutional Encoder $\quad 57$

4.2.1.2 Polynomial Representation of Convolutional Encoding $\quad 58$ 
4.2.1.3 State Diagram : $\quad 59$

4.2.1.4 Trellis Diagram $\quad 60$

4.2.2 Convolutional Code Decoding $\quad 61$

$\begin{array}{ll}\text { 4.2.2.1 Viterbi Decoding Algorithm } & 62\end{array}$

4.2.3 Matlab Implementation of the $(2,1,4)$ Convolutional Code $\quad 66$

$\begin{array}{ll}\text { Chapter 5: WCDMA } & 67\end{array}$

$\begin{array}{ll}\text { 5.1 WCDMA Technology } & 67\end{array}$

5.1.1 Direct Sequence Spread Spectrum $\quad 68$

$\begin{array}{ll}\text { 5.1.2 Code Division Multiple Access } & 70\end{array}$

5.1.2.1 Walsh Orthogonality $\quad 73$

5.2 Matlab Implementation of WCDMA

$\begin{array}{ll}\text { Chapter 6: RESULTS } & 80\end{array}$

$\begin{array}{lr}\text { Conclusion } & 92\end{array}$

$\begin{array}{ll}\text { References } & 94\end{array}$

Appendix A: Matlab Simulation Files $\quad 96$

Appendix B: Matlab Example Files $\quad 135$ 


\section{List of Figures}

Figure 1: Source-Channel Codec for a WCDMA Based Multimedia System. 3

Figure 2: Frequency Spectrum of the Signal 'speech.wav'. 15

Figure 3: Signal 'speech.wav' in Time Domain. 16

Figure 4: Grey Level Image of a Diagonal Black Line. $\quad 20$

Figure 5: PDF function of the Black Diagonal Line. 22

Figure 6: A Huffman Tree. $\quad 26$

Figure 7: Single Parity Check Code. $\quad 42$

Figure 8: Rectangular Parity Code $(I=4, J=6)$. 43

Figure 9: Decoding Table for Linear Block Codes. 48

Figure 10: $(2,1,4)$ Convolutional Encoder. 55

Figure 11: Trellis Diagram Representation of $(2,1,4)$ Convolutional Encoder. $\quad 60$

Figure 12: The Decoder Trellis Diagram (with Hamming Distances). 64

Figure 13: The Decoder Trellis Diagram after $\mathrm{t}=5$ Time Units. 65

Figure 14: Direct Sequence Spread Spectrum. $\quad 68$

Figure 15: Right Shifted Generator Polynomial. $\quad 69$

Figure 16: Speech Data. $\quad 80$

Figure 17: Still Image Data. $\quad 80$

Figure 18: Frequency Spectrum of Speech Signal. $\quad 81$

Figure 19: PDF of Quantization Error. $\quad 81$

Figure 20: Frequency of Usage of Quantizer Levels. $\quad 83$

Figure 21: PDF of the Image. $\quad 84$

Figure 22: BER vs. SNR for $(12,8)$ Linear Block Code. $\quad 88$

Figure 23: Received Speech Data.

Figure 24: Received Image. 


\section{List of Tables}

Table 1: Generator Polynomials for Convolutional Codes. 56

Table 2: Impulse Response of the $(2,1,4)$ Encoder. $\quad 57$

Table 3: State Diagram of the $(2,1,4)$ Convolutional Encoder. 59

Table 4: Humming Distance Used for Decoding Convolutional Codes. 61 


\section{Chapter 1: INTRODUCTION}

Even though nowadays personal computers are the dominant Internet access client, mobile phones and handheld devices will very soon become the major source of Internet connections. Unlike the first-generation (IG) of mobile communication systems designed mostly to carry the voice application traffic, the third-generation (3G) of communication systems promises unparalleled access in ways that have never been possible before. Internet access, voice communication over Internet protocol, and transmission of still and moving images are just a few of the communication techniques used in the "always-on" type of access that $3 \mathrm{G}$ developers have been working on. These developers envision the picture of an ordinary user receiving live music, conducting interactive web sessions, and having simultaneous voice and data access with multiple parties at the same time. Undoubtedly, this kind of access requires a special technology so that computers, handheld devices, or any other appropriately geared communication device, may all be connected anytime, anywhere. Wideband code division multiple access (WCDMA) technology is one of the main technologies for the implementation of the third-generation of communication systems that allows very high-speed multimedia services to be performed. WCDMA will support high rate high quality data, multimedia, streaming audio, streaming video, and broadcast type services among users. In addition, WCDMA designers contemplate that broadcasting, mobile commerce, games, interactive videos, and even virtual private networking will be possible in a near future, all from small portable devices.

These new and exiting possibilities of connecting people, businesses, and even industries all over the world by using rapidly developing technologies and standards make impossible to imagine what modern living would be like without access to reliable, economical, and efficient means of communication. Consequently, an ordinary person may get easily confused and scared away with concepts of all these communication techno logies and their implementations. However, the sole purpose of communications has not changed much since the time of Guglielmo Marconi who, in 1897, had demonstrated radio's ability to provide continuous contact with ships sailing the English channel. Even today, a communication is still a way of conveying or transmitting information from one place to another. The definition sounds simple and does not tell us much about what is involved in information transmission as a mean of communication between two or more parties. The answer would probably turn out to be more complicated then it might first appear and will, in fact, form a basis for this project. 
A simple answer would be that the transmission of information requires some kind of signals in order to convey a message to the other party. The signal could be a voltage, but once the voltage is established there is not much availability to convey information unless we change the value of the voltage. The next step would be to attach the voltage (a battery) to a variable resistor creating more variations of possible voltages that in addition allows us to associate more parts of information with different voltage levels. If a signal varies with time $(120 \mathrm{~V}, 60 \mathrm{~Hz}$ sinuso idal voltage) then variations of the signal may be created by either changing the amplitude or the phase of the sinusoidal voltage.

At this point we will make a rough aberration between two types of signals that could be used in a process of information transmission. If a signal is a continuous electrical signal that varies with time, it is considered to be an analog signal. On the other hand, if a signal is non-continuous, it is said to be a digital signal. Furthermore, analog signals can take on any value from an infinite set of values in a specific range while digital signals take on one of two possible amplitude levels called nodes. Digital signals consist of pulses or digits with discrete levels or values. The value of the signal is specified as one of two possibilities such as either I or 0 , high or low, true or false and so on.

In the process of transmitting information, all signals bearing information are contaminated by noise. Noise is generated by many natural and man-made events that introduce errors during the transmission and create serious problems for the party receiving the information to be able to properly recover transmitted messages. When an analog signal is affected by noise it is much more difficult to regenerate that signal than it is to recover a signal that is of the digital origin. When affected by noise a pulse (representation of a digital signal) degrades as a function of line length. Before the pulse is degraded to an ambiguous state, it could be amplified by a digital amplifier that recovers the pulse's original shape. In the case of analog signals, once an analog signal is distorted, the distortion can never be completely removed by the process of amplification.

Besides being more resentful to noise, there are many other features that make a digital signal more suitable to convey information compared to an analog signal. Some of them are: reliability of the system, flexibility of the hardware, and pricing of the implementation. On the other hand, a digital transmission would typically require a greater system bandwidth to communicate the same information in a digital format as compared to those in an analog format. All this makes a digital signal to be a signal of choice for the communication when information is to be transmitted in a reliable, robust, and relatively not expensive manner. 


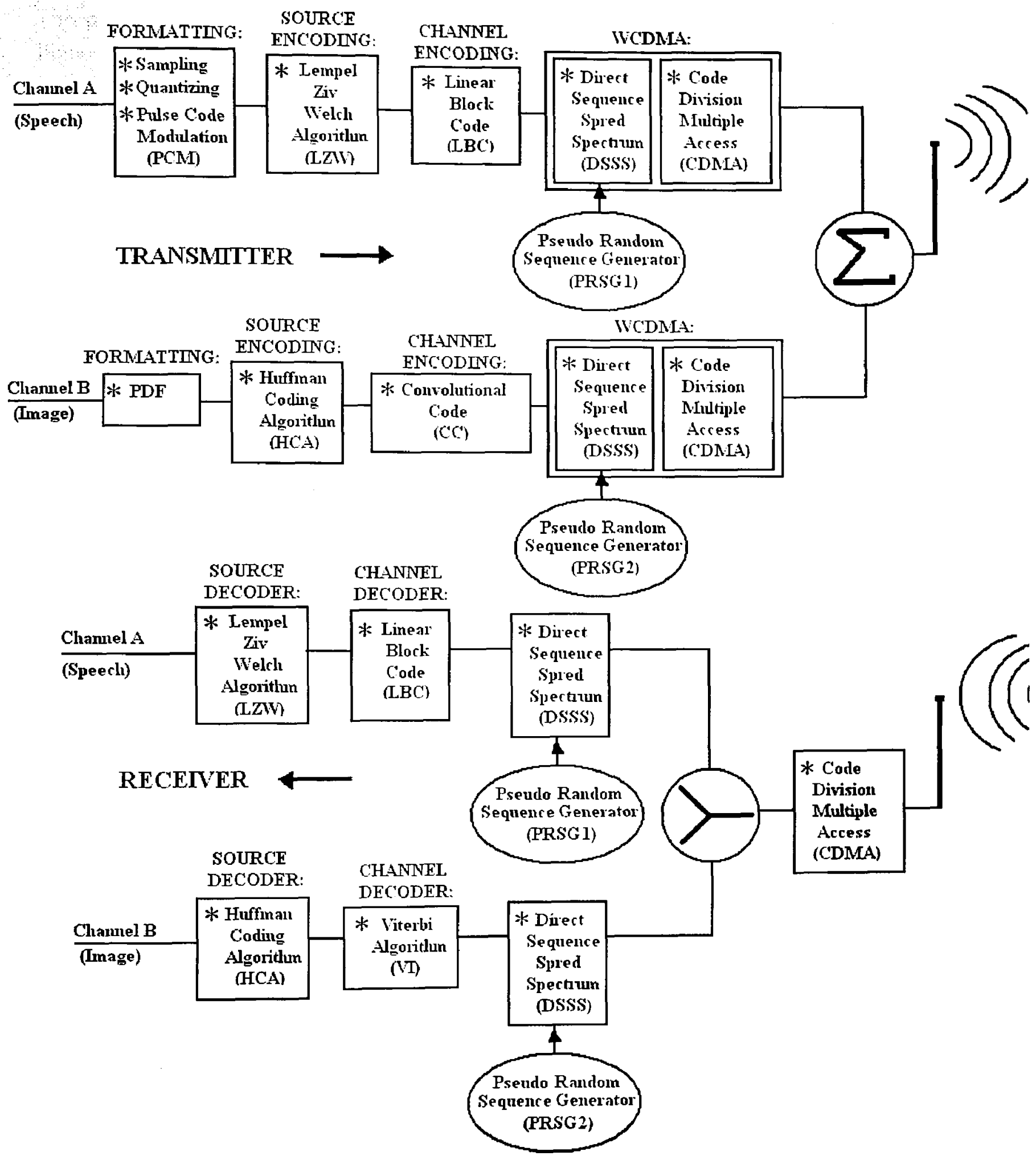

Figure 1: Source-Channel Codec for a WCDMA Based Multimedia System. 
This project simulates the basic elements of a digital communication system shown in Figure 1. Keeping in analogy with the introduction given in the beginning of this report, this system may be considered as a primitive system of the third generation (3G) of communication systems. Here are the reasons:

a) It is a digital system; meaning that regardless of the format of the input message, the system will conventionalize the message into a stream of binary digits. An exception applies only in the case when the input message is already in the digital format. This process of transforming any form of input message into a stream of bits (digital form) is called formatting and will be discussed in details in Chapter 2;

b) It is a multimedia system; meaning that it processes more than one type of media at the same time (speech and still image). The system is two channeled, where channel $\mathrm{A}$ is carrying a speech data while channel $\mathrm{B}$ is dealing with a still image data. In addition, the system is considered to be a simplex transmission system. In many cases it is desirable to maintain two way communications, or at least be able to send a message back to its origin for possible verification, comparison, or control. To simply implement this type of system, called full-duplex, another set of blocks (stages) exactly the same ones as the ones shown in Figure 1 should be incorporated into the system but in a reverse order; That second set of blocks would be responsible for the transmission of information from the destination back to the source;

c) It uses WCDMA technology in the process of transmitting data from the source to the destination. At this point, it would be beneficial to say that WCDMA is more than just a technology; it is a standard that establishes and defines various communication protocols and procedures used during a communication session. In this project many of these protocols and procedures will be ignored for the sake of simplicity. However, one that is considered as the main representative of WCDMA standard, called Medium Access Control (MAC), will be fully presented and simulated. Medium access control defines how digital signals from different sources use the same allocated frequency spectrum to convey different information to different recipients. MAC is discussed in Chapter 5;

After formatting, the next block in our digital communication system is the source encoder stage. The source encoding is the process of removing redundant bits from the 
sequence of bits carrying information. In other words, the source encoding compresses data in a way that only necessary hits that make up the original information are processed further through the system. In Chapter 3, two source encoding techniques are demonstrated. One for the channel A that deals with the source encoding of a speech signal formatted into a stream of bits in the previous stage (formatting stage). That source encoding technique is called the Lempel-Ziv-Welch technique. The other technique called Huffman Coding Algorithm will be used for the channel B to encode the still image data that is, at this point in the process, already in the binary form. The chapter will also introduce the ways how data encoded by the two encoding techniques can be decoded on the receiver end. Since a source encoder and source decoder generally operate in pairs, this combination of a source encoder and decoder is called a source codec (coder-decoder).

The stage following the source encoding stage is the channel encoding stage. The purpose of the channel encoder is to introduce, in a control manner, some redundancy in the binary information stream that can be used at the receiver to overcome the effects of noise and interference encountered in the transmission of the bits through the channel. In other words, the system will now add some redundant bits to the binary information for the purpose of detecting and correcting some of errors occurred during the transmission. The redundant bits are formed and organized in such a way that no response back from the receiver is needed in order to detect and correct errors. This type of error control is called forward error correction (FEC) and requires a one-way link only.

In Chapter 4, two types of channel encoding techniques are presented. One, for channel A, called the Linear Block Code technique and the other one, for the channel B, called the Convolutional Code technique. Along with both techniques their associated decoding procedures, such as Viterbi decoding algorithm for Convolutional codes, will be discussed and implemented. Similarly as for a source encoder and decoder, a channel encoder and decoder operates in pairs as well, thus forming what is called a channel codec.

Chapter 5 entirely deals with the access control of the medium and, as it has earlier been mentioned, presents the way of managing this issue as defined by the WCDMA standard (technology). A digital modulation technique called Direct Sequence Spread Spectrum is presented along with the Code Division Multiple Access technique. The role of each one of them in the channelization process is discussed. 
The last chapter, Chapter 6 , provides an overview of the results obtained from the simulation in such a way that the input/output binary sequences to/from each block in the systems are examined and discussed. The chapter also gives a conclusion on the project as well as recommendations on how to improve the efficiency and efficacy of the system presented and simulated in this project.

For the end of this introductory section, I would also like to mention one important thing that would become more evident once a reader starts reading through the incoming pages. Numerical examples! Throughout my entire education as an undergraduate as well as a graduate student I had always felt that any engineering theory or topic introduced in a class would have made much more sense to me if it was accompanied by a relevant example. That is why at the end of each chapter in this report an example associated with the algorithm or technique presented in that chapter is worked out. I believe that these examples, besides clarifying the theory, will also help a reader to understand how the presented algorithm or technique is implemented in Matlab. 


\section{Chapter 2: FORMATTING}

\subsection{Speech Formatting}

In general, signals in the communication theory could be considered either as analog or digital ones. Signals in digital form are also told to be discrete signals. However, both types of signals bear information that is conveyed from the source to the destination through different types of medium(s) (e.g. air, copper wire, etc.). Besides analog and digital types of information mentioned above, the data at the source, also called source information, may be found in a textual form. Such kind of source data is termed as textual information.

In the digital communication systems the first and essential step in the process of conveying information from the source to the destination is to format the source information regardless of the form that information is in. That essentially means that the source information has to be processed in a certain way that will make it suitable for further digital processing. Let's consider all three possible forms of source information that have been mentioned earlier and the way they are formatted in order to be compatible with the next stages in a digital communication system.

The smallest headache would give us an information source, or data, that is already in the digital form. It will simply just bypass the formatting stage and proceed to the next processing step. The problem begins with the fact that the most data in communications is either in textual or in analog format. Data in textual format would usually be encoded with one of several standards such as: ASCII (American Standard Code for Information Interchange), EBCDIC (Extended Binary Coded Decimal Interchange Code), Baudot, and Hollerith. The aforementioned standards transform the textual data into a digital format.

Now, we come to ana log information at the source and we are interested how it can be made suitable for further digital processing. Simply said, we would like to determine the necessary conditions which will allow us to change analog information to digital one without loss of information. As a criterion of how well the process of converting the analog information into the digital one can be carried out, we use an important condition that the original information can be fully reconstructed by using reversible, digital to analog processing steps. 
In essence, analog information is formatted using three separate steps: sampling, quantization, and coding.

\subsubsection{Sampling}

The link between an analog signal and the corresponding digital signal is given by what is known as the sampling theorem. The sampling theorem states the following: A real valued band limited signal having no spectral components above a frequency of $\mathrm{f}_{\mathrm{m}}(\mathrm{Hz})$, known as the maximum frequency of the analog signal, is determined uniquely by its values at uniform intervals spaced no grater than $T_{S}$ seconds apart, where $T_{S}$ is:

$$
\mathrm{T}_{\mathrm{s}}=\frac{1}{2 \cdot \mathrm{f}_{\mathrm{m}}} .
$$

This statement is a sufficient condition that an analog signal can be reconstructed completely from a set of uniformly spaced discrete samples in time. The output of the sampling process is called the pulse amplitude modulation (PAM) because the successive output intervals can be described as a sequence of pulses with amplitudes derived from the samples of the analog signal. If equation stated in (1) is applied, all replicas of the original spectral density are just tangent to each other and an ideal low pass filter can be used to reconstruct (theoretically) the original analog signal from the sampled version of that signal. However, if the sampling interval $T_{S}$ becomes slightly larger than the right side of the equation (1), then there will be an overlap of spectral densities and the original signal will not be successfully reconstructed from its sampled version with the help of an ideal low pass filter. In order to avoid the situation described in previous sentence it is essential and absolutely necessary in the process of sampling of an analog signal that:

$$
\mathrm{T}_{\mathrm{s}}<\frac{1}{2 \cdot \mathrm{f}_{\mathrm{m}}} .
$$

The equation given in (2) is a mathematical interpretation of the sampling theorem. The maximum time interval $T_{S}$ is called the Nyquist interval. If we want to see the equation (2) as the relationship between the maximum frequency of the analog signal $f_{m}$ and the sampling frequency $f_{S}$, where $f_{S}$ is reversely proportional to $T_{S}$ then we arrive to the following equation:

$$
f_{s} \geq 2 \cdot f_{m}
$$


The equation (3) given in terms of the sampling and maximum frequencies is called the Nyquist Sampling Rate. In practice, the full potential of the sampling theorem usually cannot be realized and the equations (2) and (3) serve as upper bounds on actual performance. One reality fact that we are faced with in dealing with the sampling theorem is that we cannot build an ideal low pass filter. We can only build a low pass filter with as fast an attenuation rate as possible. One thing that we can do to overcome the inability to have an ideal low pass filter is to increase the sampling frequency to allow some frequency space before the next frequency replica of the sampled analog signal appears.

Another reality fact being responsible for the sampling theory not being used in its full potential is the fact that a time limited signal is never strictly band limited. When such an analog signal is sampled, there will always be some unavoidable overlap of spectral components. Furthermore, in reconstructing the sampled version of the signal, frequency components originally located above one half of the sampling frequency will appear below this point and will be passed by the low pass filter. This is known as aliasing and results in a distortion of the signal. The effects of aliasing can be partially eliminated by applying as good as possible low pass filtering before sampling and by sampling at rates greater than the Nyquist rate.

An interesting question arises here; if we want to apply the sampling theorem to bandpass signals, do we still have to obey the rule given by the equation (2), stating that we have to sample bandpass signals at twice the highest frequency? The answer would be no, since the minimum sampling rate depends on the bandwidth of a low pass signal rather then on its highest frequency. In the case of low pass signals these two conditions coincide. However, when sampling a bandpass signal we should use a minimum sampling rate in the range between 2 and 4 times the bandwidth of the signal. This minimum rate requirement for bandpass signals approaches the limit of twice the bandwidth as the center frequency of the signal increases.

\subsubsection{Quantization}

After the sampling, there comes the second step in formatting of an analog signal; quantization. Quantization or quantizing is the task of mapping samples of an analog signal, obtained through the process of sampling, to a finite set of amplitudes. To make an analogy with the sampling process, the process of quantization is related to the $y$ ordinate similarly as the process of sampling is related to the $\mathrm{x}$-axis. The simplest quantizer performs mapping of each sample of the sampled analog signal to one of the 
predetermined quantizer levels. If those predetermined quantizer levels are equally spaced then we say that the quantizer is a linear quantizer. Similarly, if the levels are not equally spaced then we say that the quantizer is a nonlinear quantizer. Since this project deals with the linear quantization only, the further discussion on the process of the quantization of a sampled analog signal will be strictly limited to a linear quantizer and its characteristics. However, it is important to make a comment that the nonlinear quantization provides much better the signal to noise ratio (SNR) than the linear quantization does. This is particularly evident in speech communication where very low speech volumes predominate $70 \%$ of the time.

A linear quantizer is the universal form of the quantizer in a sense that it makes no assumption about the amplitude statistics and correlation properties of the input analog signal. The only two conditions that have to be known in order to implement a linear quantizer are: the dynamic range of the sampled signal (DR) and the number of bits that each sample is represented. The dynamic range is defined as:

$$
\mathrm{DR}=\left[\max \_ \text {sig }-\min \_ \text {sig }\right],
$$

where 'max_sig' and 'min_sig' are the maximum and minimum values of the sampled analog signal. The second condition, the number of bits that each sample in the sampled version of the analog signal will be represented with, is directly related to the number of levels of the desired quantizer. The relation between the number of bits for each sample representation and the number of levels of the quantizer is given by:

$$
\mathrm{L}=2^{\mathrm{R}} \text {. }
$$

In equation (5) $\mathrm{L}$ is the number of the levels of the quantizer and $\mathrm{R}$ is the number of the bits that each sample is represented with. Now, by taking a logarithm with the base 2 to each side of the equation (5), we get another relation between the number of bits for each sample representation and the number of levels of the quantizer:

$$
\mathrm{R}=\log _{2} \mathrm{~L}
$$

At this point it is essential to reveal a restriction and an observation associated with the process of quantization. The restriction is that the equations (5) and (6) are valid only if we intend to apply the fix length representation of each sample. In the case of variable length representation, the equations (5) and (6) are no longer valid. More overview and discussion on fixed and variable length representations of samples will be given later in 
Chapter 3, when we deal with the Huffman code algorithm. The observation to be revealed is that once quantized, the instantaneous values of the analog signal can never be exactly reconstructed again.

Once we establish a desired number of levels for the quantizer, we can easily determine the size of each quantizer level, usually called the quantile interval. A remainder that a quantizer has all its quantile intervals of the same size only if that quantizer is a uniform quantizer. The size of each quantile interval $q$ is given by the following equation:

$$
\mathrm{q}=\frac{\left(\max \_\operatorname{sig}-\min \_ \text {sig }\right)}{\mathrm{L}} .
$$

A linear quantizer works in a very simply way. Inputs to the quantizer are samples of an analog signal obtain through the process of sampling, while the outputs from the quantizer are predetermined values of the quantizer's levels that the samples are mapped to. The difference between the input and output of the quantizer is called the quantization error. Mathematically, the quantization error is represented as:

$$
\mathrm{e}(\mathrm{n})=\mathrm{x}(\mathrm{n})-\tilde{\mathrm{x}}(\mathrm{n})
$$

where $x(n)$ represents the input vector containing the signal samples, $x(n)$ is the quantized output vector, while $e(n)$ is the error vector. The quantization error vector is also referred to as the quantization noise. Under the restriction that the input signal has a smooth probability density function (PDF) over the quantization interval, it can be assumed that the quantization errors are uniformly distributed over the quantization interval ranging from $-q / 2$ to $q / 2$. Each probability density function must be greater or equal to zero and must satisfy the following condition:

$$
\int_{-q / 2}^{q / 2} f(x) \cdot d x=1
$$

In order to satisfy the equation (9), the probability density function of the quantization error $p(e)$ must be equal to $1 / q$ in the interval from $-q / 2$ to $q / 2$. Outside that given interval $p(e)$ must be equal to zero. Here is mathematically interpretation of what has just been said: 


$$
p(e)=\left\{\begin{array}{cc}
\frac{1}{q} & \text { for }-\frac{1}{q} \leq e \leq \frac{1}{q} \\
0 & \text { otherwise }
\end{array}\right.
$$

A useful figure of merit for a uniform quantizer is the error variance, where the error variance is defined as:

$$
\sigma^{2}=\int_{-q / 2}^{q / 2}\left(e-m_{x}\right)^{2} \cdot p(e) \cdot d e
$$

where $m_{x}$ is the error mean that is equal to:

$$
m_{x}=\int_{-q / 2}^{q / 2} e \cdot p(e) \cdot d e=\int_{-q / 2}^{q / 2} e \cdot \frac{1}{q} \cdot d e=\frac{1}{q} \cdot\left|\frac{e^{2}}{2}\right|_{-q / 2}^{q / 2}=0 .
$$

Now, by placing the error mean $\mathrm{m}_{\mathrm{x}}$ from the equation (12) into the equation (11) we get the error variance:

$$
\sigma^{2}=\int_{-q / 2}^{q / 2} e^{2} \cdot p(e) \cdot d e=\frac{1}{q} \cdot\left|\frac{e^{3}}{3}\right|_{-q / 2}^{q / 2}=\frac{q^{2}}{12} .
$$

The error variance is also commonly known as the noise power. Similarly, the signal variance (signal power) is defined as:

$$
\sigma_{\mathrm{s}}=\int_{-\infty}^{\infty} \mathrm{x}^{2} \cdot \mathrm{p}(\mathrm{x}) \cdot \mathrm{dx}
$$

and can be substituted with the expression for the peak power of an analog signal normalized to $1 \Omega$ :

$$
P_{s}=V_{s} \cdot I_{s}=V_{s} \cdot \frac{V_{s}}{R_{s}}=V_{s}^{2}=\left(\frac{V_{p p}}{2}\right)^{2}=\left(\frac{L \cdot q}{2}\right)^{2}=\frac{L^{2} \cdot q^{2}}{4}
$$


The ratio between the signal variance (signal power) given in the equation (15) and the noise power given in the equation (13) yields the quantization signal to noise ratio, $(\mathrm{SNR})_{\mathrm{q}}$ :

$$
(\mathrm{SNR})_{\mathrm{q}}=\frac{\text { signal power }}{\text { noise power }}=\frac{\frac{\mathrm{L}^{2} \cdot \mathrm{q}^{2}}{4}}{\frac{\mathrm{q}^{2}}{12}}=3 \cdot \mathrm{L}^{2}
$$

The $(\mathrm{SNR})_{\mathrm{q}}$ is usually given in the units of decibels $[\mathrm{dB}]$ and is obtained by applying the following conversion formula:

$$
(\mathrm{SNR})_{\mathrm{q}}[\mathrm{dB}]=10 \cdot \log _{10}(\mathrm{SNR})_{\mathrm{q}}
$$

From the equation (16) we conclude that the signal to noise ratio for a uniform quantizer solely depends on the number of levels L. Since from the equations (5) and (6), the number of levels $\mathrm{L}$ is directly proportional to the number of bits used to represent each sample, thus we can also say that the signal to noise ratio depends on the number of bits used to represent each sample. The more bits are used to represent a sample, the better the signal to noise ratio. It is easily proven that for each additional bit used to represent a sample (e.g. increase from a 5-bit sample representation to a 6-bit sample representation), the improvement in the $(\mathrm{SNR})_{\mathrm{q}}$ is approximately about $6 \mathrm{~dB}$. A quick approximation of the (SNR) $)_{q}$ for a quantizer is to multiply the numbers of bits that each sample is represented with by $6 \mathrm{~dB}$. However, the real quantization $(\mathrm{SNR})_{\mathrm{q}}$ is much smaller due to the imperfections in the quantizer itself (linear vs. nonlinear quantizer). As the number of levels L approaches infinite, the signal approaches to its form before the quantization (PAM format) and the (SNR) $)_{q}$ approaches to infinity. In other words, with the infinite number of quantization levels, ultimately there is no quantization noise.

\subsubsection{Pulse Code Modulation (PCM)}

The next step in the process of converting an analog signal into a digital one is to assign a digital value for each quantile interval in such a way that each interval has a one to one correspondence with the set of real integers. This is called the digitization. The process of digitization reduces the original analog signal to a set of digits, at the successive sample times, mapped into L quantizer's levels. Each sample of the original analog signal is assigned a quantization level (quantile interval) closest to the value of the 
actual sample. The digits are expressed in a coded form. The most common code used for this purpose is a binary code where each digit is represented as a combination of zeros and ones. Each binary 1 is further represented by a pulse and each binary 0 is represented by the absence of a pulse. Thus, instead of transmitting the individual samples, a combination of zeros and ones, binary pulse code, is sent at each sample time carrying the intended information in digitized form. Communications systems making use of this kind of data representation during the transmission are commonly called pulse code modulation systems; PCM systems.

Waveform carrying information can be transmitted even more efficiently if we represent them as sequences of transitions between upper and lower voltage levels. When the waveform is at the upper voltage level it represents a 1 . Similarly, when the waveform is at the lower voltage level it conveys a 0 . There are many PCM waveform types classified in many groups. Here only a few will be mentioned: Non Return to Zero (NRZ), Return to Zero (RZ), Phase Encoded, and Multilevel binary. The most commonly used are NRZ PCM waveforms. The reason why there are so many different types of PCM waveforms lies in differences in performances for different waveform coding schemes. Some schemes are better in performing error detections, some are better in correcting data errors, some schemes again are better in increasing the efficiency of bandwidth utilization. Certain types of PCM waveforms are more immune than others to noise. All this contributes that so many of PCM waveform schemes are used, but the decision which one and when to be used greatly depends on required performances and characteristics of the digital system used.

\subsubsection{Matlab Implementation of Formatting}

The formatting process explained in preceding sections is simulated in Matlab with three M files: 'Sampling.m' and 'Quantization.m' files that describe the sampling and quantization processes and 'PCM.m' file that deals with the process of digitization, where each quantized sample is coded to a corresponding binary word. In the 'Sampling.m' file an analog signal in the form of a sound file ('speech.wav') is loaded in the Matlab environment. Matlab provides the command 'wavread' which performs sampling automatically when the sound file is loaded into the environment. The standard sampling rates for $P C$ based audio hardware are $8,000,11,025,22,050$, and 44, 100 samples per second. Mono signals are returned as one column matrix, while stereo signals are returned as two column matrices. The first column of a stereo audio matrix corresponds to the left input channel, while the second column corresponds to the right input channel. The 'wavread' command, after being executed, returns two output 
variables: the sampled data and the sample rate. The sample rate used for the speech file used in this project was $22,050 \mathrm{~Hz}$. Let's see how the sampling frequency is obtained.

If we plot the frequency spectrum of the speech signal as shown in Figure 2 we see that the maximum frequency of the signal is $11,025 \mathrm{~Hz}$. Now, if we recall the equation (3) stating that the Nyquist Sampling Rate must be equal or greater than the maximum frequency of the signal, it is clear why the sample rate that our signal is sampled with is $22,050 \mathrm{~Hz}$.

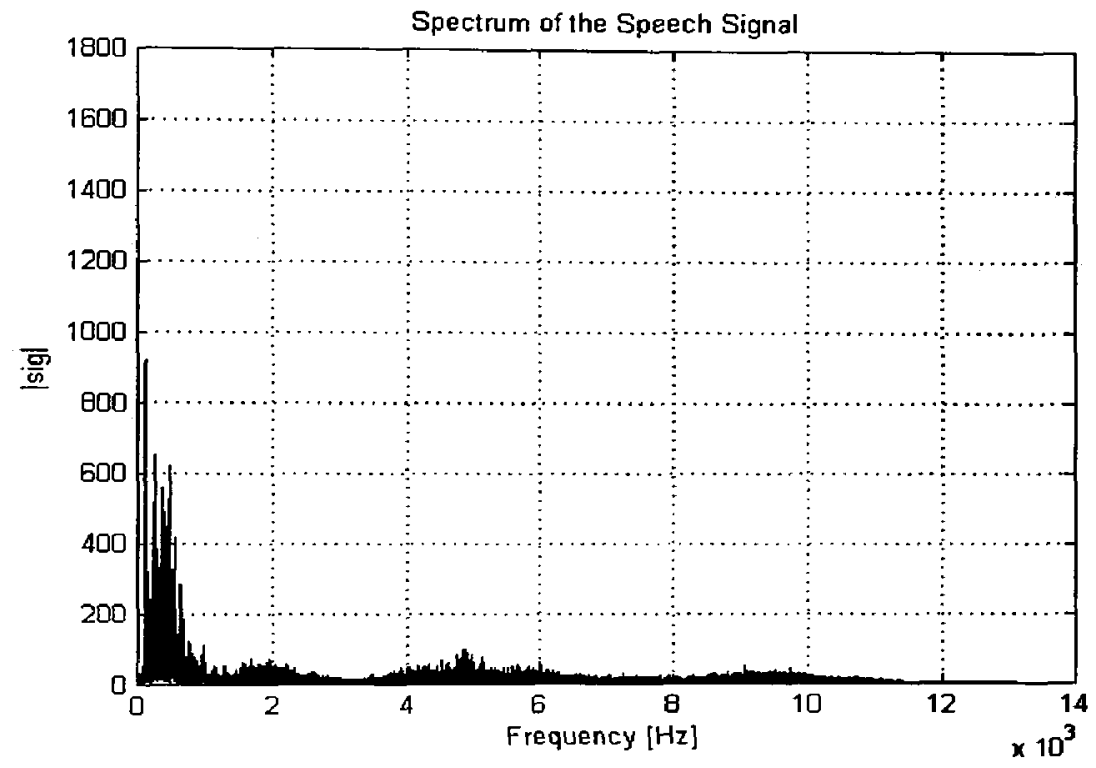

Figure 2: Frequency Spectrum of the Signal 'speech.wav'.

The sampled data contains 110,033 samples. That number can be obtained through a simple calculation where the number of samples is equal to the product of the duration of the signal (in seconds) and the number of samples for 1 second. We already know that the number of samples for I second is equal to the sampling frequency, which is 22,050, and we can conclude from the Figure 3, which shows the ASCII representation of the speech signal, that the duration of the speech signal is 4.9902 seconds. A simple calculation gives us the number of samples that the original speech signal is represented after the sampling process:

$$
4.9902 \times 22,050=110,033 \text { samples. }
$$




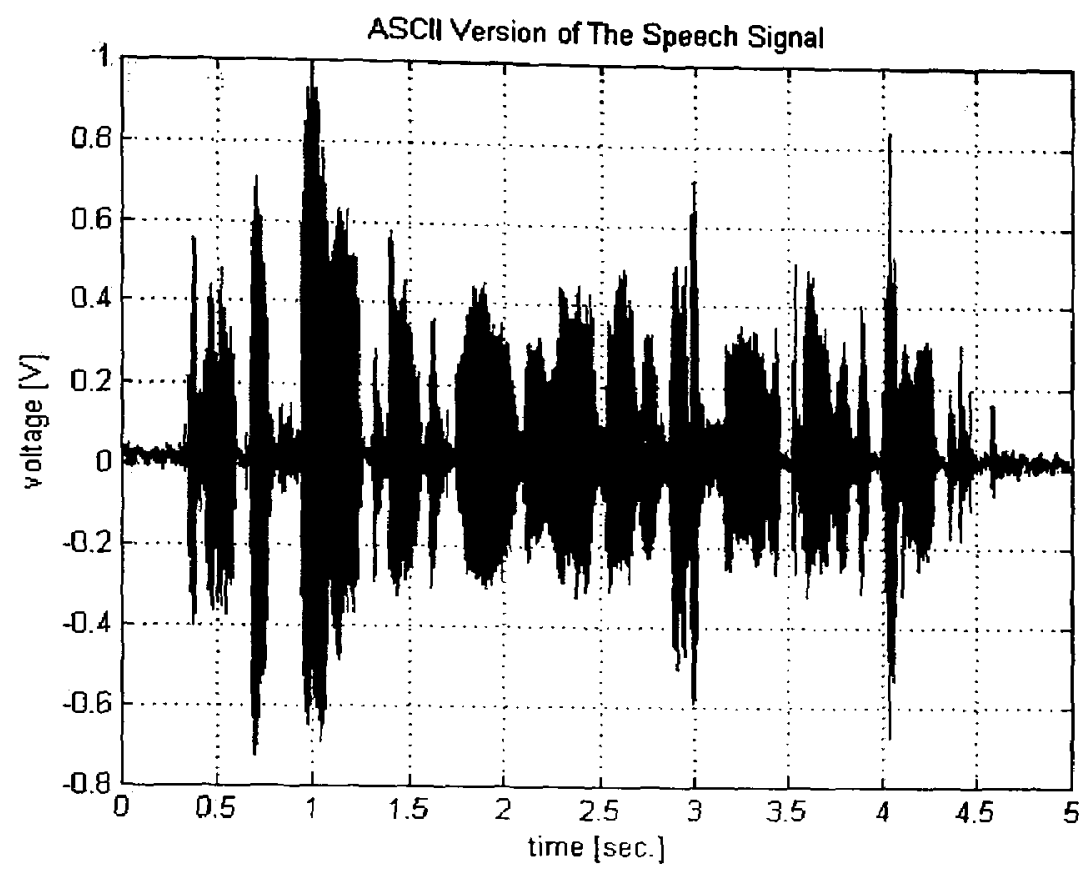

Figure 3: Signal 'speech.wav' in Time Domain.

In the 'Qunatizing.m' file, as per earlier discussion (the equations (4), (5), and (6)), we firstly have to specify the dynamic range of the sampled signal as well as the number of levels that our quantizer will have. The Matlab commands, 'max' and 'min', allow us to obtain the dynamic range of the sampled signal (see the equation (4)). The number of levels $\mathrm{L}$ is chosen to be 256 since we deal with a speech signal. Speech signals in general require 8 bits per sample since it is the minimum number of bits that can allow us to hear original speeches in via their quantized versions. Applying the equation (5), we see how the number of quantization levels is obtained.

Now when we know the dynamic range of the sampled signal and the number of levels for the quantizer, we can calculate the quantization step $q$ that is also the size of the quantile interval. Once again, since we deal with a uniform quantizer each one of 256 quantile intervals will have the same size. To obtain the value of $q$ we simply apply the equation (7).

Upon its execution, the Matlab command 'quantiz' produces as the output two variables for each sample of the sampled signal; the quantization index 'indexI' and quantized output value 'quants1':

[index1, quants1] =quantiz (sig,partition1, codebookl), 
where 'partitionl' is a real vector whose elements are values of 256 quantizer's levels assigned to each sample. The elements of the 'partitionl' vector must be given in strictly ascending order. The input variable 'codebook 1 ' is a vector codebook that prescribes a value for each partition in the quantization and its length exceeds the length of the 'partitionl' vector by one. As mentioned earlier, the output variables are: 'indexl' and 'quantsl'. If the 'partitionl' vector has the length of $\mathrm{n}$, then the 'indexl' vector is a column vector whose $k^{\text {th }}$ entry is given by:

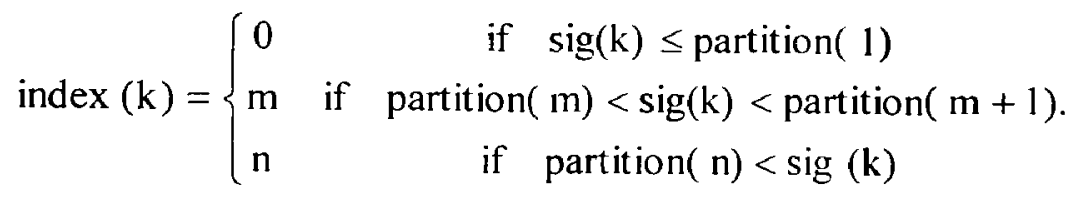

The output variable 'quants 1 ' is a row vector whose length is the same as the length of the input sampled signal. The row vector 'quantsl' contains the quantization of the sampled signal based on the quantization levels and prescribed values. The 'quants1' is related to the 'codebook 1' and 'indexl' variables by the following equation:

$$
\text { quants }(\mathrm{k})=\operatorname{codebook}(\text { index }(\mathrm{k})+1) \text {. }
$$

In this equation, $\mathrm{k}$ takes on integer values between 1 and the length of the sampled signal. The variable 'index 1 ' contains elements (decimal numbers) that represent the membership of each sample to one of 256 quantization levels. In other words, those decimal numbers represent which level $L$ each sample has been assigned to. Any number from 0 to 255 is a valid number that a sample can be assigned to. The task of 'PCM.m' Matlab file is to convert the decimal index number, indexl(k), of each sample to a related code word. Since code words are supposed to be in the binary form, thus each code word is created as a combination of binary ones and zeros.

The conversion from decimal to binary is performed by the 'convert2binary. $m$ ' Matlab file. The code does the following: It takes each element of the 'indexl' vector and keeps dividing the value by 2 until the result is 1 , keeping the track of remainders for each division. When 1 is reached as a result of dividing, the remainders are appended to the resulting 1 , forming the binary representation of the decimal value. Here is a quick example:

Let's convert the number 109 to its binary representation: 


$$
\begin{aligned}
& 109: 2=54(1), \\
& 54: 2=27(0), \\
& 27: 2=13(1), \\
& 13: 2=6(1), \\
& 6: 2=3(0), \\
& 3: 2=1 \quad(1) .
\end{aligned}
$$

The resulting binary representation of the number 109 is: 1101101 . Here is also the result obtained by the Matlab file ' $d 2 b . m$ ' file, where the ' $d 2 b . m$ ' file is the modified version of the 'convert2binary' and can be used separately from the main 'PCM.m' file that performs the process of digitization (' $\mathrm{d} 2 \mathrm{~b} . \mathrm{m}$ ' is listed in the Appendix B section of this report):

ans $=1 \quad 1 \quad \begin{array}{llllll}1 & 0 & 1 & 1 & 0 & 1 .\end{array}$

There is now one more thing that has to be taken care of. In the equations (5) and (6) we have given the relation between the number of levels of the quantizer and the number of bits that each code word should be composed of. Since we use a 256 level quantizer in this project, by applying the equation (6) we obtain that each code word should consist of 8 bits. However, we know that only the numbers from 128 to 255 need 8 bits for their binary representation. So, what happens with the numbers from 0 to 127 that do not need all 8 bit for their binary representations (e.g. the number 109 needs 7 bits, see the example above). Well, to have each number in the range from 0 to 255 represented by 8 bits, we have to add the so-called 'missing' bits. For instance, the number 109's binary representation is a 7 bit combination of ones and zeros: 110110 1. To obtain a 8 bit binary representation we have to concatenate one 0 at the beginning, so that the resulting 8 bit representation is: 0111011101 . The file 'convert2binary.m' takes care that every number in the $[0,255]$ interval is represented with 8 bits creating socalled a fixed length representation of code words.

At this point we also have to create a stream of bits made up of the binary representation (code word) for each symbol in the information that is to be transmitted. The code words are place in the stream in the FIFO manner, meaning that the code word for a symbol that is to be sent out first is placed at the beginning of the stream, the second code word is appended onto the first one and so on until all symbols from the information are processed. 


\subsection{Image Formatting}

Matlab stores a gray level image in the form of a two-dimensional matrix, where each element of the matrix corresponds to a single pixel in the displayed image. For example, an image composed of 200 rows and 300 columns of differently colored dots would be stored in Matlab as a 200 by 300 matrix. Some images, such as RGB (RedGreen-Blue) images, require a three-dimensional matrix to be stored in, where the first plane in the three dimensions represents the red pixels' intensities, the second plane represents the green pixels' intensities, and the third plane represents the blue pixels' intensities.

If there is a need to convert a gray level image into a color level image, a straight forward conversion is used. That is exactly what has been done in this project. A color image is converted into gray level image in order to save computational time as well as memory space. That means that instead of processing three two-dimensional matrices for a color image, we will be processing only one 2-dimensional matrix for a gray level image. Memory wise that means that instead of using 3 bytes of memory storage for each colored pixel, we will need only 1 byte to store the number represent ing one pixel. The conversion will be carried by using the following formula:

$$
\overline{\mathrm{A}}=0.2989 \cdot \overline{\mathrm{I}}(:,:, 1)+0.5870 \cdot \overline{\mathrm{I}}(:, ;, 2)+0.1140 \cdot \overline{\mathrm{I}}(:, ;, 3) .
$$

In the equation (18), $\overline{\mathrm{A}}$ is a 2-dimentional matrix representing a gray level image, $\overline{\mathrm{I}}(: ;, ;)$ is the 'Red Intensity' part of the color image, $\bar{I}(:, ;, 2)$ is the 'Green Intensity' part of the color image, and $\overline{\mathrm{I}}(:, ;, 3)$ is the "Blue Intensity" part of the color image. The coefficients that the intensities are multiplied with in the equation (18): $0.2989,0.5870$, and 0.1140 are related to the eye's sensitivity to a Red, Green, and Blue color according to the NTSC (National Television System(s) Committee) standard and are obtained throughout an experimental way.

As mentioned earlier each pixel in a gray level representation (2-dimentional matrix) will be represented by 8 bits. This means that intensity of each pixel will be in the range of 0 to 255 , where 0 represents the pure black color, while 255 represents the solely white color. This can be shown by a simple Matlab program that displays a 7 by 7 gray level image representing a black diagonal line as pictured in Figure 4: 


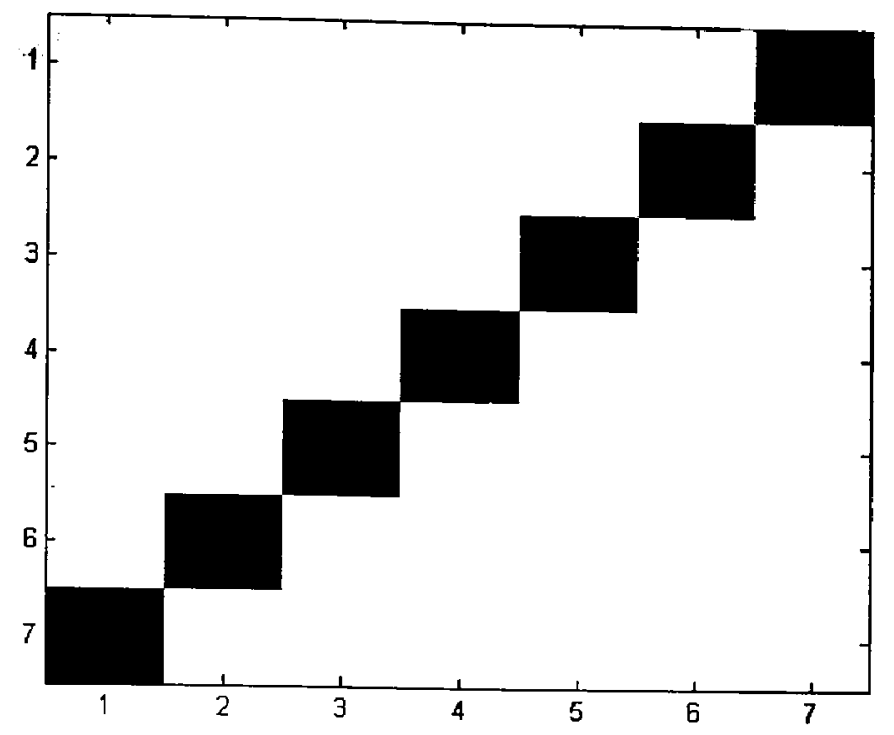

Figure 4: Grey Level Image of a Diagonal Black Line.

Here is the simple Matlab code that displays the image shown in Figure 4:

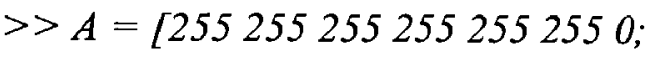

$$
\begin{aligned}
& 2552552552552550255 \text {; } \\
& 2552552552550255255 \text {; } \\
& 2552552550255255255 \text {; } \\
& 2552550255255255255 \text {; } \\
& 2550255255255255255 \text {; } \\
& 0255255255255255255] \text {; }
\end{aligned}
$$

$>>$ image $(A)$;

$>$ colormap (gray(256));

$>>$ imwrite $(A$, 'diag_line', 'JPG');

$>A=$ imread('diag_line','JPG');

At the first double-prompt line a 2-dimensional matrix $\bar{A}$ of the size $7 \times 7$ (49 pixels) is created where the all 255 numbers represent pure white intensity of the pixels, while the all 0 numbers represent pure black intensity of the pixels. Next, the matrix is displayed using Matlab 'image' command. The 'colormap(gray(256))' command sets the color map of the image to black and white (gray level image). The 'imwrite' command saves the image as 'diag_line.jpg' file. If we now want to import the file 'diag_line.jpg' into the Matlab environment for any further processing and manipulation, we would use 
the 'imread' command and the black diagonal line image will be imported back into the $\overline{\mathrm{A}}$ matrix. The above demonstrated procedure is exactly how the image in this project is formatted by using Matlab environment.

A natural question that a curious person might ask at this point is: how do we convert the ASCII representation of the image (a matrix containing the numbers in the [0-255] range) to its equivalent binary interpretation? Recall that we have earlier said that in order to process any information through a digital communication system, we have to have the information being represented (so is our image) by a combination of zeros and ones.

The answer is that we will produce a normalized probability density function (PDF) of our image, calculate the probability of occurrence of each number in the [0-255] range and then use the Huffman coding algorithm to obtain the binary representation of the image. In the process of calculating the probability of occurrence for each number in the [0-255] range, any number in the range that does not appear in the image matrix is considered as irrelevant as if it carries no information relevant to our image. All those 'irrelevant' numbers will be removed from the list of leaves that are supplied to the Huffman code algorithm for data compression. Once the frequency of occurrence of each number in the matrix is calculated, it is divided with the total number of elements in the matrix forming the probabilities of occurrence for each number in the [0-255] range that appears in the matrix.

For example, if we consider the matrix $\bar{A}$ containing pixels of the $7 \times 7$ black diagonal line image from Figure 4, we see that the number 255 occurs 42 times, while the number 0 occurs only 7 times. Dividing the ir frequencies of occurrences with the total number of elements in the matrix, 49, we obtain the corresponding probabilities of occurrence for the numbers 0 and 255 :

Probability of occurrence for $0: 7 / 49=0.1428=14.28(\%)$, Probability of occurrence for $255: 42 / 49=0.8571=85.71(\%)$.

The corresponding PDF function of the black diagonal line would look as shown in Figure 5. In the project, the Matlab file, 'Formatting.m', performs the formatting of a color image 'world.jpg'. The file is written in exactly the same manner as being explained above: it performs the RGB to gray conversion (equation (18)), calculates PDF of the image, and gives, for the next stage in the process, two vectors; one that contains all numbers present in the image, and the other one that contains the respective probabilities of occurrence for all numbers in the range [0-255] present in the image. 


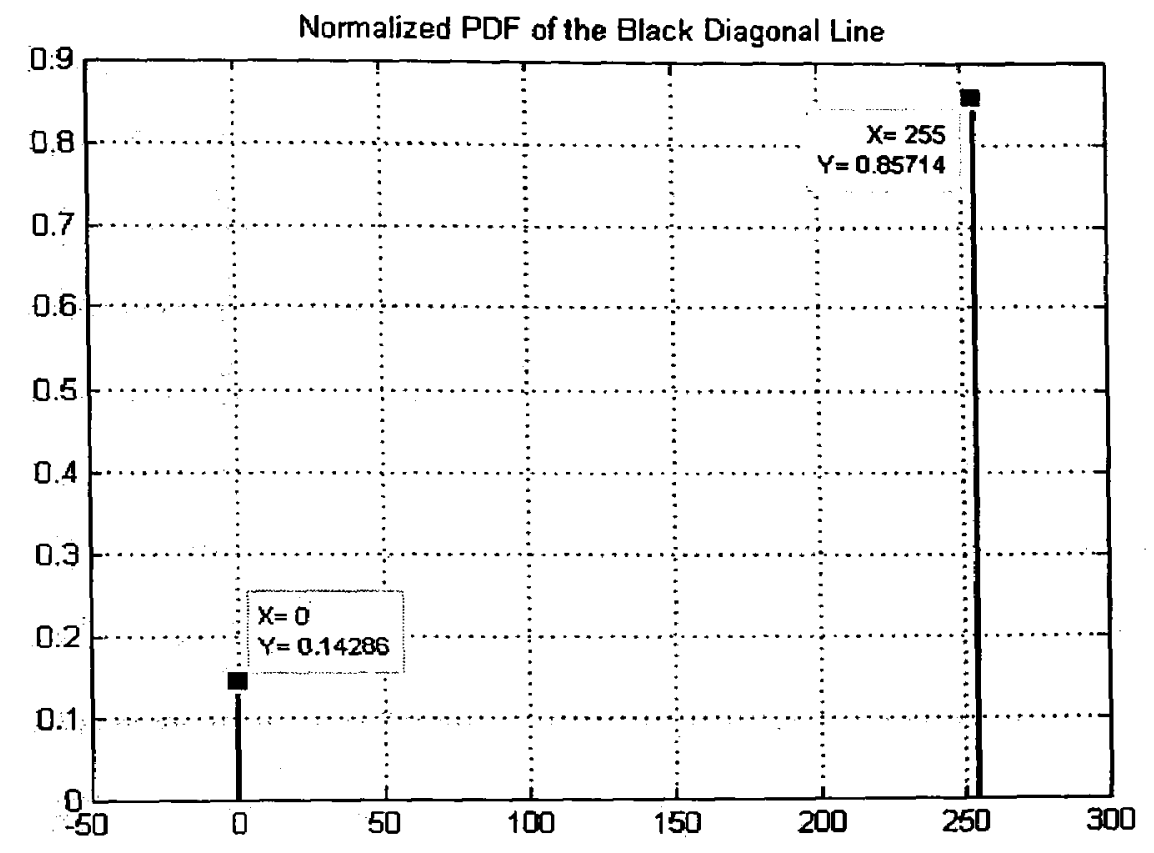

Figure 5: PDF function of the Black Diagonal Line. 


\section{Chapter 3: SOURCE CODEC}

\subsection{Huffman Coding Algorithm}

Before starting a discussion on the Huffman coding algorithm, some basic terms and definitions needed for understanding the principles of the Huffman algorithm will be first presented. We will start with the definition of a finite discrete source, where a finite discrete source is a discrete source that is emitting, at a constant rate, a finite number of symbols from the source alphabet called a stream of symbols. Each symbol in an infinite long (theoretically) stream emitted by the source is assigned its own probability of occurrence $P$. Once we know the probability of a symbol, we can introduce the concept of the information, where the information I is defined as a value inversely proportional to the probability of occurrence of the letter $P$ :

$$
\mathrm{I} \propto \frac{1}{\mathrm{P}}
$$

More precisely, the information content of a symbol $x_{i}$ is defined as a base 2 logarithm taken from the probability of occurrence of the symbol $x_{i}$. Mathematically:

$$
\mathrm{I}\left(\mathrm{x}_{\mathrm{i}}\right)=\log _{2} \frac{1}{\mathrm{P}\left(\mathrm{x}_{\mathrm{i}}\right)}=-\log _{2} \mathrm{P}\left(\mathrm{x}_{\mathrm{i}}\right) \text {. }
$$

Since the source alphabet contains a finite number of symbols $\mathrm{N}$, we would be more interested in finding a measure of the information content for all the symbols in the source alphabet, rather than dealing with the information content of each symbol independently. Such a measure of the information content for all the symbols in the source alphabet is called the entropy of the source and is defined as the average amount of information emitted by the source:

$$
H(X)=\sum_{i=1}^{N} P\left(x_{i}\right) \cdot I\left(x_{i}\right)=-\sum_{i=1}^{N} P\left(x_{i}\right) \cdot \log _{2} P\left(x_{i}\right) .
$$

The unit for the entropy is a bit per event and should not be confused with a binary digit called a bit. If all symbols in the source alphabet are equally probable (have the same probabilities of occurrence), then the entropy of such a source is given by the following equation: 


$$
H(X)=\log _{2} N \text {, }
$$

where $\mathrm{N}$ is a finite number of symbols in the source alphabet.

Now, we will consider the process of encoding, or representing each symbol in the source alphabet by a sequence of binary digits; ones and zeros. Since there are $\mathrm{N}$ possible symbols in the source alphabet, the number of binary digits $\mathrm{R}$ per symbol required to uniquely encode each symbol is given by:

$$
\mathrm{R}=\log _{2} \mathrm{~N} .
$$

The equation (23) holds only when the number of symbols in the source alphabet is a power of 2 . If it is not the case, the equation (23) requires a minor adjustment and is rewritten as:

$$
\mathrm{R}=\left\lfloor\log _{2} \mathrm{~N}\right\rfloor+1,
$$

where the symbol, $L\rfloor$, identifies the largest integer less than ' $\log _{2} N$ '. The efficiency of the encoding is defined as the ratio between the entropy $H(X)$ and the number of binary digits $\mathrm{R}$ needed to represent any of $\mathrm{N}$ possible symbols in the source alphabet. The binary representations of $\mathrm{N}$ equally probable symbols are called the fixed-length code words.

On the other side, when the source symbols do not have the same probability of occurrence, as it is the case in the real world, a different encoding method is required. An example of such an encoding scheme is the Huffman coding algorithm. Huffman coding algorithm is a variable-length encoding algorithm based on the probabilities of the source symbols. The algorithm is considered as an optimum algorithm in the sense that the average number of bits required to represent the source symbols is minimum. In other words, the objective of the algorithm is to provide a systematic procedure for constructing uniquely decodable variable-length code words that are efficient in the sense that the average number of bits per source, defined as the following quantity:

$$
\bar{n}=\sum_{i=1}^{N} P\left(x_{j}\right) \cdot n_{i},
$$


is minimized. It is absolutely necessary that these variable-length code words are uniquely and instantaneously decodable. They also have to satisfy the pretix condition that requires that for a given code word $C$ of length $k$, no other code word of length $\mathrm{m}$, where $\mathrm{m}>\mathrm{k}$, contains the code word $\mathrm{C}$ as its prefix.

The measure of efficacy of the variable-length encoding is the ratio between the source entropy $H(X)$ and the average length of the code word $\bar{n}$, and is represented by the Greek letter $\eta$ :

$$
\eta=\frac{H(X)}{\bar{n}}(\%), \quad 0 \leq \eta \leq 1
$$

The equation (26) tells us that the entropy of the source can not be greater than the average length of the code word. Based on this, we can present another equation that gives the relation between the source entropy and the average length of the code word:

$$
H(X) \leq \bar{n}<H(X)+1 .
$$

\subsubsection{Huffman Encoding}

Let's now take a look how the coding algorithm works. The Huffman coding algorithm that generates variable-length code words is considered to be a tree forming process. The process starts by listing all letters in the source alphabet, along with their probabilities in descending order of occurrence. These entries correspond to the leaves (ends) of the tree. Each leaf is assigned a leaf weight equal to the probability of occurrence of that leaf. The two leaves with lowest probabilities of occurrence are merged together forming a branch whose weight will be equal to the composite probabilities of the merged leaves. After each merging, the new branch and the remaining branches (or leaves) are reordered to assure that the reduced tree preserves the descending order of the probability of occurrences for all current braches and remaining leaves.

Here we distinguished two different ways of reordering the remaining branches: the top and the bottom approach. To demonstrate these two approaches, let's assume that a newly forming branch has the composite probability equals to 0.25 , and that during the process of reordering the branches in the tree we find that another two branches have the same probabilities of occurrence of 0.25 as the newly formed branch does. The top approach puts the newly formed branch on the top of the branches with the same 
probability. On the other side, the bottom approach places the newly branch at the bottom of the 0.25 probability group. The process of merging the two branches with the lowest probabilities and then reordering all branches in the descending order according to their probabilities of occurrence continues until last two branches are merged together producing a root of the tree whose probability of occurrence is equal to 1 .

Once we end up with the complete tree (obtaining a root with the probability of occurrence equals to 1), all the upper branches of the tree are labeled $w$ ith a 0 and all the lower branches are labeled with a 1 . A Huffman tree in Figure 6 demonstrates the procedure just explained:

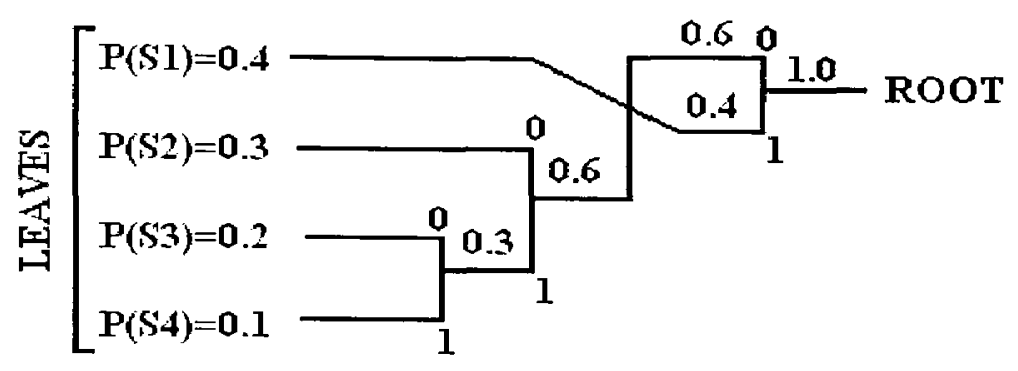

Figure 6: A Huffman Tree.

The code word for each symbol in the source alphabet (leaf) is a sequence of zeros and ones obtained by traveling the tree in the direction starting from the root and ending at the leaf whom the code word is being formed for. Here are the code words for the Huffman tree shown in Figure 6:

$$
\begin{aligned}
& S_{1}=1 \\
& \mathbf{S}_{2}=00 \\
& \mathbf{S}_{3}=010 \\
& \mathbf{S}_{\mathbf{4}}=011
\end{aligned}
$$

The best way to understand the Huffman coding algorithm is to go throughout an entire example that will emphasize all steps of the algorithm: the merging, the top approach reordering, the entropy, the average length of code words, and the encoding efficiency:

\section{EXAMPLE (Huffman Coding Algorithm):}

$A$ discrete memoryless source (DMS) has an alphabet of eight symbols with the following probabilities: $0.25,0.2,0.15,0.12,0.1,0.08,0.05$, and 0.05 . 
a) Determine binary code words for the source output;

b) Determine the entropy of the source;

c) Find the average code length;

d) Determine the efficiency of the encoding;

SOLUTION:

a)

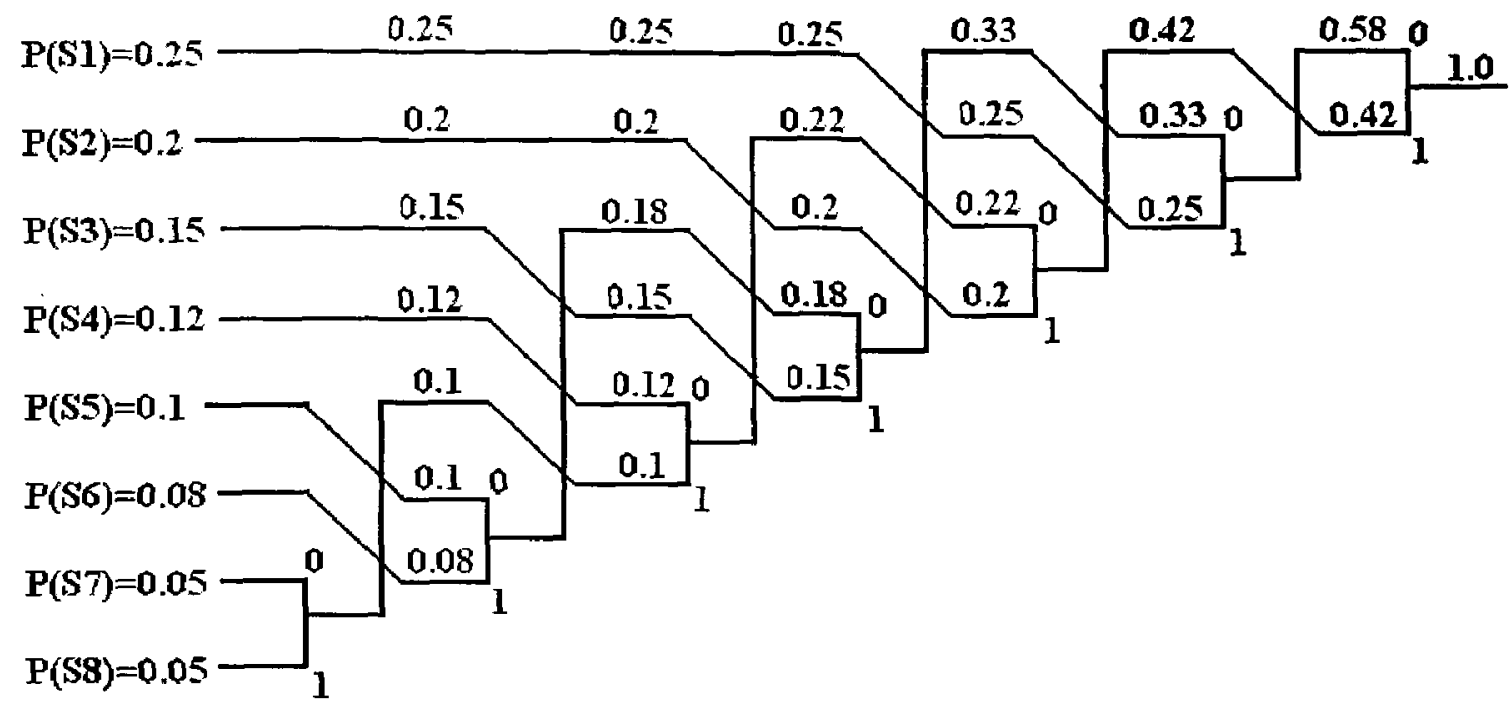

$S_{I}=01$

$S_{2}=11$

$S_{3}=001$

$S_{4}=100$

$S_{5}=00000$

$S_{6}=0001$

$S_{7}=1010$

$S_{8}=1011$

b)

$H(X)=\sum_{i=1}^{8} P\left(x_{i}\right) \cdot I\left(x_{i}\right)=0.25 \cdot 2+0.2 \cdot 2.32+0.15 \cdot 2.74+0.12 \cdot 3.06+0.1 \cdot 3.32+$ $+0.08 \cdot 3.64+0.05 \cdot 4.32+0.05 \cdot 4.32=2.7979$ 
c)

$$
\begin{aligned}
\bar{n}=\sum_{i=1}^{8} n_{i} \cdot P\left(x_{i}\right) & =2 \cdot 0.25+2 \cdot 0.2+3 \cdot 0.15+3 \cdot 0.12+4 \cdot 0.1+4 \cdot 0.08+4 \cdot 0.05+4 \cdot 0.05 \\
& =2.83
\end{aligned}
$$

d)

$\eta=\frac{H(X)}{\bar{n}}=\frac{2.7979}{2.83}=0.9886=98.86(\%)$

\subsubsection{Huffman Decoding}

Earlier, it was mentioned that it was absolutely necessary for variable-length code words to be uniquely and instantaneously decodable. We will use an example to demonstrate what it means. Below are shown three code assignments for representing the

\begin{tabular}{|c|c|c|c|}
\hline Symbols & Code I & Code 2 & Code 3 \\
\hline $\mathrm{S}_{1}$ & 1 & 0 & 0 \\
\hline$S_{2}$ & 0 & 01 & 10 \\
\hline $\mathrm{S}_{3}$ & 00 & 011 & 110 \\
\hline $\mathrm{S}_{4}$ & 11 & 01111 & 111 \\
\hline
\end{tabular}
symbols $\mathrm{S}_{1}, \mathrm{~S}_{2}, \mathrm{~S}_{3}$, and $\mathrm{S}_{4}$ :

Let's now determine which codes are uniquely and instantaneously decodable and which are not. Suppose we are presented with the following sequence to be decoded:

$$
010001111001 \ldots
$$

Using Code 1 it is clear that the first two symbols correspond to $S_{2} S_{1}$. The next two bits are not uniquely decodable. They can be decoded either as $\mathrm{S}_{2}$ or $\mathrm{S}_{3}$. Obviously this code is not unique and therefore is not useful for the purpose of decoding. The second code, Code 2, will decode the first four bits as $\mathrm{S}_{2} \mathrm{~S}_{1} \mathrm{~S}_{1}$. The problem begins with the bits $5,6,7$, and 8 . Would the bits 5 and 6 correspond to $S_{2}$, or we have to wait for the bit 7 and decode the sequence (bits 5,6 , and 7) as $S_{3}$, or we have to wait even more for the bit 8 to decode the new sequence (bits $5,6,7$, and 8 ) as $S_{4}$ ? Code 2 is not instantaneously decodable and can not be used for decoding. Now, Code 3 will decode the given sequence as $S_{1} S_{2} S_{1} S_{1} S_{4} S_{2}$ and so on. It is uniquely and instantaneously decodable. Note 
that no code word in this code is a prefix of any other code word. This is exactly what helps us decode a stream of bits at the receiver side.

There are several methods to decode sequences previously encoded by the Huffman coding algorithm. For all of these methods one thing is in common; the decoder must have some kind of knowledge about the encoding procedure in order to work properly. One way is to send the list of all symbols and their counts to the receiver so the receiver can recreate the Huffiman tree on its end. Then when a sequence to be decoded arrives, one bit in the sequence is read at a time and the Huffman tree is traveled from the root down until a leaf is reached. Let's apply this method using the tree from Figure 6. We assume that the tree is reconstructed at the decoder's end and that the following sequence has arrived:

$$
010000111010 \ldots
$$

The first bit, 0 , is read and the tree will be traveled in the upward direction. Since the node we have arrived at is not a leaf node, we go on and take the next bit, l. We now travel the tree in the downward direction. Once again, we check if the reached node is a leaf node. It is not, so we take the next bit, 0 , and travel upwards. This time we have reached a leaf node, the leaf $S_{3}$. We decode the first three bits as the symbol $S_{3}$ and go back to the root of the tree to start decoding the next symbol. Similarly, we take the next 2 bits (one at a time) and reach the leaf $S_{2}$. The process continues until we run out of bits in the arriving sequence.

Another method is to store the code words for each symbol in different structures (tables) according to the length of the code words. In other words, all code words that are 5 bits long will be stored in the table 5, for example. Those with the length of 7 will be stored in the table 7 and so on. We also create a so-called general table which contains the code words with the length of $1,2,3$, and 4 bits (since there are not too many of them). All these tables are then sent to the decoder. Now, when a stream of bits arrives at the decoder, each bit in the stream is read at a time and the cumulative subsequence (could be formed from 1 or more bits) is search for in the appropriate table. If a corresponding symbol is found, we have the decoded symbol. If not the next bit is appended to the subsequence. This method is used in this project.

The most interesting decoding method (but also the most time consuming method) is socalled the adaptive Huffman tree decoding. In this method we do not have to have the knowledge about the probabilities of occurrence of the symbols. All we have to know is 
how many symbols we could have in the source alphabet. That information is shared with the decoder. That is all that has to be transmitted to the decoder. Thus, instead of sending the list of all symbols and their counts or the tables with the code words, all we send is a simple number that represents how many symbols the source has in the alphabet. The next step is that both the transmitter and the receiver assign all the symbols' counts to 1 . That results in having the same probabilities of occurrence for all the symbols.

For example, if we want to compress a human speech we will need an 8-bit quantizer generating 256 possible output levels. So, the number of symbols in the source alphabet is 256. This is sent to the receiver and both the transmitter and receiver set the counts for each of 256 symbols to 1 that generates the same probability of occurrence for each symbol $(1 / 256=0.003906)$. Now, the code words are generated using the Huffman algorithm on both ends. For example, when a sample that is quantized to the level 56 is to be transmitted, the transmitter firstly, sends the code word that corresponds to the symbol 56 to the receiver, secondly, increments by one the count of the symbol 56 which automatically increases the probability of occurrence of the symbol 56, and thirdly, regenerate the new code words using Huffman algorithm for all the symbols according to their new probabilities of occurrence. At this point the transmitter is ready to process a new quantized sample.

The receiver, on the other end, firstly, decodes the received code word (to 56) by comparing it with the existing code words at the receiver, secondly, increments by one the count of the symbol 56, and thirdly, re-generates the new code words using Huffman algorithm for all the symbols. After this the receiver is ready to accept the next code word.

We see that during the procedure of encoding and decoding the code words are constantly changing and that is why this method is called the adaptive or dynamic Huffman coding. The ordinary Huffman discussed in the most of this chapter is in some books called the static Huffman coding.

\subsubsection{Matlab Implementation of Huffman Coding Algorithm}

Three Matlab files implement the Huffman Coding Algorithm. The Matlab file "Image_Huffman.m" is the main program that contains calls for the routines called at various times during the execution of the program. It performs the merging of two leaves with lowest probabilities of occurrences forming a new branch whose weight is equal to 
the composite probabilities of the leaves. It also calculates the entropy, the average length code, and efficiency of the encoding.

The file "MakeStructHuff.m" is the Matlab function that creates a cell "Huff with the following structure elements: "probability' that keeps the values of probability for each source symbol, 'codew' which keeps the binary ones and zeros obtained at various stages of the Huffman tree process. At the end of the process the structure 'codew' contains the code words for all the source symbols, 'symbols' containing the rearranged original position of each letter assigned to each source letter at the beginning of the Huffman tree procedure;

The file "Sorting_Huff.m" is the Matlab function that keeps the order in the Huffman tree. It makes sure that at the beginning of the execution of the algorithm, the probabilities of the source symbols are arranged in a descending order. Also, this routine is called every time when a compound branch is formed in the tree (from the 2 branches that have the lowest probabilities of occurrence);

The last file "Decoder_Image_Huffman.m" is the Matlab file that decodes a sequence of code words using the tables containing all possible code words. These tables are created according to the lengths of the code words. They must be sent to the receiver before the process of decoding may begin.

Here is how the code works: At the beginning, the "MakeStructHuff.m" routine is called to create a cell (structure) in order to allocate the necessary memory space for holding the probabilities of all the source symbols, their original positions in the source alphabet, and a 2-dimentional matrix that keeps the binary ones and zeros assigned to each source symbol while the code travels throughout the Huffman tree. Once the cell is created, the main program calls the "Sorting_Huff.m" routine that check the order of the probabilities all the elements in the 'Huff.probability' structure. If necessary, it rearranges the existing order of the probabilities to obey the descending order requirement. Now, the main program takes from the 'Huff.probability' the two symbols with the lowest probabilities and creates their compound probability. The process repeats itself until only one compound probability is left (equal to 1). The decoding procedure is straight forward and is explained into details in the previous section of this chapter.

At the end of the Huffman code algorithm discussion, let's take a look at the Matlab print out to the Huffman algorithm program. The same input sequence of the source symbols 


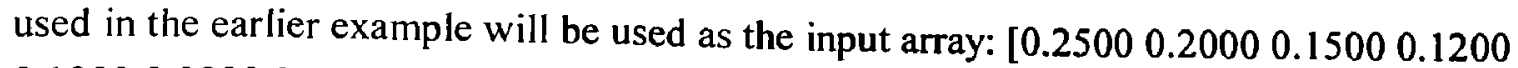
$\left.\begin{array}{llll}0.1000 & 0.0800 & 0.0500 & 0.0500]\end{array}\right]$

>> HuffCodeAlgorithm

$S 1=01$

$S 2=11$

$S 3=001$

$S 4=100$

$S 5=0000$

$S 6=0001$

$S 7=1010$

$S 8=1011$

Average Code Length (using my code): 2.83

Entropy of the Source $H(x)=2.7979$

Efficiency $=98.865 \%$

Huff $=$

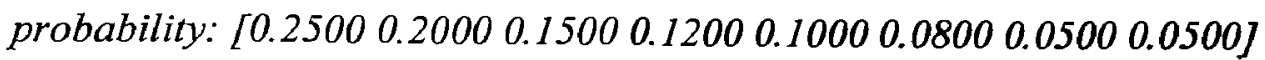

codew: [8x8 double]

symbols: [8x8 double]

sorted_Huff $=$

probability: 1.0000

symbols: [8x1 double]

It can be easily observed that the Matlab code has produced the same output of code words as the example demonstrated earlier. The entropy of the source, the average code length, as well as the efficiency match those obtained in the example.

\subsection{Lempel-Ziv-Welch Algorithm}

Unlike the static Huffman coding where we must know the probabilities of occurrence for all source symbols, the Lempel-Ziv-Welch compression algorithm does not require any prior knowledge of occurrence of source symbols. This is similar to what goes on in the real world, where the statistics of sources are very often unknown.

Does th is mean that we can not design a Huffman code for a source for which we do not know the statistics of occurrence of the source symbols? Theoretically the answer is a yes 
but practically the answer would be a no (adaptive Huffman coding is extremely time consuming). Furthermore, theoretically it can be done by estimating the probability of source symbols through observing a long information sequence emitted by the source and then empirically obtaining the probabilities of all the source symbols. However, this would be very impractical since the process of empirically obtaining those probabilities would be computationally very complex. Not to even mention the complexity of Huffman coding algorithm being applied onto sources with memories. Thus, the application of the Huffman code algorithm for many real sources is generally considered impractical and a different method of source encoding is needed. This is where the Lempel-Ziv-Welch algorithm comes into the picture.

The Lempel-Ziv-Welch algorithm for the source encoding is designed to be completely independent of the probabilities of occurrence of source symbols. Due to this fact the algorithm is placed in the class of algorithms called the universal source coding algorithms. The algorithm works in a following manner; the output sequence from the source is parsed into variable-length blocks called subsequences. A new subsequence is created every time when a sequence of bits comes out of the sources and has not been introduced in any previously defined subsequence. The subsequences are then listed in a Lempel-Ziv-Welch table in the order of appearance. Besides having a column in which all subsequences are listed, the subsequence column, the Lempel-Ziv-Welch table contains three other columns: the memory location column, the memory representation column, and the code word column.

The memory location column contains entries that are binary representation of the decimal numbers listed in ascending order starting from 1 and representing the appearance of the different subsequences. As we have earlier said the subsequence column contains the subsequences where the later subsequences take lower positions in the subsequence column. The fields (positions) in the memory location column and subsequence column are mutually related in a sense that every new subsequence introduced has the memory location number associated with it being greater by 1 than the memory location number of the last known subsequence. The very first obtained subsequence occupies the third position in the subsequence column (having the memory location of 3) since the positions (memory locations) 1 and 2 are reserved for subsequences 0 and 1 that are usually assumed even before the source starts emitting bits. The last bit of each subsequence entered in the subsequence column is called the innovation bit and is important for creating the code words that will be placed in the code word column. Before considering how the code word column is filled, let's first consider how we obtain the entries for the memory representation. In order to do that, we will 
assume that the source has just started emitting a stream of binary ones and zeros. The assumption goes on assuming that the very first two bits emitted are a 1 and 0 , creating a new subsequence (recall that a 0 and 1 are considered as the first two subsequences occupying the first two positions in the subsequence table). Our new subsequence 10 is assigned the memory location 011 (binary representation for the decimal number 3) and respectively the position 3 in the subsequence column. The last bit of the subsequence 10 is the bit 0 and is considered, as we have previously said, to be the innovation bit.

To obtain the memory representation for the subsequence 10 , we divide the subsequence in two groups. The first group contains $n-1$ bits (where $n$ is the total number of bits in the subsequence), while the second group contains only one bit, the innovation bit. In our case the first group forms bit 1 ( since $n=2$ ), while the innovation bit, 0 , forms the second group. At th is point we will also mention that the first two subsequences 0 and 1 do not usually have their respective memory representations in the memory representation column. Now, we take the first group, the bit 1 and look for its exact replica among previously introduced subsequences. This means that we would look for subsequence 1 among the $\mathrm{m}-1$ previous entries in the subsequence column, where $\mathrm{m}$ is the position in the memory representation table that the current subsequence holds (in our case $m=3$ ). So, we will look for the subsequence 1 in the positions 1 and 2 in the subsequence column. We find it at the position 2 and that is the first number of the memory representation of the subsequence 10 . We repeat the same procedure for the innovation bit and find it at the position 1 . That represents the second number of the memory representation for the subsequence 10 . Now, those two numbers are concatenated forming the number 21 and this is the number being the memory representation of the subsequence 10 .

The last column in the Lempel-Ziv-Welch table is the code word column. A code word representation for a sequence is obtained from its memory representation along with the value of its innovation bit. To demonstrate this, we will still stick with our subsequence 10 whose innovation bit is 0 and its memory representation is 21 . The code word is obtained on the following way; the memory representation entry 21 is divided into two groups. The first group is formed from $k-1$ numbers, where $k$ is the total number of numbers in the memory representation entry. In our case $k=2$ and the first group contains the number 2 . The second group would contain the last number of the memory representation entry which would be 1 . Now, the code word for the subsequence 10 would consist from the binary representation of the number(s) in the first group, in this case 2 , and the innovation bit that would be appended to it. It is here important to mention that we have to know in advance how many bits the binary representation of the 
first group would occupy. We will assume that in our example to represent the binary representation of the first group, we would use 3 bits. So, the number 2 would be converted to 010. Appending the innovation bit to it we would obtain the code word 0100 for the sequence 10 . We see that our code word is consisted from 4 bits.

Even though we have just presented how the memory location, subsequence, memory representation, and code word entries are obtained for one subsequence, the 10 subsequence, a complete example of Lempel-Ziv-Welch algorithm follows as it would be beneficial to a reader to fully understand the process of obtaining subsequences from a stream of ones and zeros emitted by the source. It would nicely round up the discussion on the Lempel-Ziv-Welch algorithm and provide us with the firm foundation for the Matlab implementation of the algorithm.

EXAMPLE (Lempel-Ziv-Welch Algorithm):

Obtain code words for the following stream of binary numbers emitted by the source: 10101101001001110101000011001. Each code word should be given in a 5-bit format.

SOLUTION:

Step_1:

Subsequences Stored (SS):

0,1

Data to be Parsed (DP):

10101101001001110101000011001

Step_2:

Subsequences Stored (SS):

$0,1,10$

Data to be Parsed (DP):

101101001001110101000011001

Step_3:

Subsequences Stored (SS):

$0,1,10,101$

Data to be Parsed (DP):

101001001110101000011001

Step_4:

Subsequences Stored (SS):

$0,1,10,101,1010$

Data to be Parsed (DP):

01001110101000011001 
Step_5:

Subsequences Stored (SS):

$0,1,10,101,1010,01$

Data to be Parsed (DP):

001110101000011001

Step_6:

Subsequences Stored (SS):

$0,1,10,101,1010,01,00$

Data to be Parsed (DP):

1110101000011001

Step_7:

Subsequences Stored (SS):

$0,1,10,101,1010,01,00,11$

Data to be Parsed (DP):

10101000011001

Step_8:

Subsequences Stored (SS):

0, 1, 10,101, 1010, 01,00,11, 10101

Data to be Parsed (DP):

000011001

Step_9:

Subsequences Stored (SS):

0, 1, 10,101,1010,01,00,11,10101,000

Data to be Parsed (DP):

011001

Step_10:

Subsequences Stored (SS):

$0,1,10,101,1010,01,00,11,10101,000,011$

Data to be Parsed (DP):

001

Step_11:

Subsequences Stored (SS):

$0,1,10,101,1010,01,00,11,10101,000,011,001$

Data to be Parsed (DP):

\begin{tabular}{cccc} 
MEMORY & MEMORY & CODE \\
LOCATION & SUBSEQUENCES & REPRESENTATION & WORD \\
\hline & & & \\
1 & 0 & - & 00000 \\
2 & 1 & - & 00001 \\
3 & $1 \underline{0}$ & 21 & 00100 \\
4 & $10 \underline{1}$ & 32 & 00111 \\
5 & $101 \underline{0}$ & 41 & 01000 \\
6 & $0 \underline{1}$ & 12 & 00011 \\
7 & $0 \underline{0}$ & 11 & 00010
\end{tabular}




$\begin{array}{llll}8 & 1 \underline{1} & 22 & 00101 \\ 9 & 10101 & 52 & 01011 \\ 10 & 00 \underline{0} & 71 & 01110 \\ 11 & 01 \underline{1} & 62 & 01101 \\ 12 & 00 \underline{1} & 72 & 01111\end{array}$

It should be noticed that the Lempel-Ziv-Welch table shown above has encoded the original 29 bit sequence into 12 code words of 5 bits each, resulting in 60 coded bits. One might ask what is the point in sending 60 bits through the channel instead of sending only 29 bits? Is it not the purpose of the source coding to remove any redundant bit from the sequence that is to be transmitted? Should not the source encoding provide some kind of compression, and in this example we have just seen that the algorithm provided no data compression at all? As a matter of fact in the latest example the algorithm has introduced an expansion of the data. The answer to all these questions is very simple; the inefficiency of the Lempel-Ziv-Welch algorithm is due to the fact that the sequence of bits we have used in our example is very short. As the sequence is containing more bits to be transmitted, the Lempel-Ziv-Welch encoding procedure becomes more efficient and results in a compressed sequence that is much shorter than the original sequence, providing a significant data compression.

The Lempel-Ziv-Welch algorithm is widely used in the compression of the computer files (e.g. ZIP application). Many so-called 'compress' and 'uncompress' utilities under the UNIX and Microsoft operating systems are nothing more than the implementations of various versions of this algorithm.

Decoding of code words that have been encoded by using Lempel-Ziv-Welch algorithm is straight forward. The transmitter must provide the table to the receiver and then each bit in the stream is read at a time and the cumulative subsequence (could be formed from 1 or more bits) is search for in the table. If a corresponding symbol is found, we have the decoded symbol. If not the next bit is appended to the subsequence.

\subsubsection{Matlab Implementation of Lempel-Ziv-Welch Algorithm}

Six Matlab files implement the Lempel-Ziv-Welch algorithm. The Matlab file "LZW_Encode.m" is the main program that contains calls for the routines called at various times during the execution of the program. The file "Subsequence.m" is the Matlab function that is doing the parsing of the "Data-to-be-Parsed" sequences into variable-length subsequences. It creates the first two columns in the Lempel-Ziv-Welch table: the memory location column and the subsequence column; 
The file "Check_up.m" is the Matlab function that checks if a chosen subsequence parsed from the "Data-to-be-Parsed" sequence has already been created in the Lempel-ZivWelch table. If it has, the subsequence is rejected from being entered into the table. "Subsequence.m" file will chose a new subsequence that will be formed from the rejected subsequence plus the next allowable bit from the "Data-to-be-Parsed" sequence. The new subsequence will be checked against the existing subsequences by the "Check_up.m" routine;

The file "Table.m" is the Matlab function that completes the rest of the Lempel-ZivWelch table creating the memory representation column and the code word column. The function "Convert2binary.m" converts the memory representation entry (minus the innovation part) into its binary representation.

The Matlab function "Tailing. $m$ " checks if the tail of the original sequence contains the bits that are left out and can not form a new subsequence (those bits are already part of the previously formed subsequence). The routine also creates an array of compressed data that is to be transmitted. The decoding process is the part of digital to analog conversion performed in the "DtoA.m" Matlab file.

In general the code works in the following manner; the main program calls the "Subsequent.m" routine that is responsible for parsing the "Data-to-be-Parsed" sequences into variable-length subsequences. The first two columns in the Lempel-Ziv-Welch table are created as well. The main program then calls the "Table.m" routine that completes the rest of the Lempel-Ziv-Welch table. At this point the Lempel-Ziv-Welch table is complete containing all four columns: the memory location column, the subsequence column, the memory representation column, and the code word column. At the end, the "Tailing. $m$ " routine creates an array of compressed data containing the binary representation of the subsequences obtained through the process of parsing.

At the end of this section, let's take a look at the output of the Matlab code that implements the LempeI-Ziv-Welch algorithm. The same input sequence used in the example above will be used as the input array in the code.

\footnotetext{
>> LempelZivWelch

$c=$
} 


\begin{tabular}{|c|c|c|c|}
\hline 1] & 0] & [] & [1x5 double \\
\hline 2$]$ & 1] & [] & [1x5 doubl \\
\hline 3$]$ & [1x2 double] & [2] & {$[1 \times 5$ doubl } \\
\hline 4$]$ & [1x3 double] & [3] & \\
\hline [ 5] & {$[1 \times 4 c$} & [4] & \\
\hline 6 & {$[1 \times 2 c$} & [1] & \\
\hline 7 & {$[1 \times 2 d$} & [1] & \\
\hline 18 & & [2] & \\
\hline$[9$ & {$[1 x$} & [5] & \\
\hline & {$[1 \times 3$} & [7] & \\
\hline [11] & & {$[6]$} & \\
\hline [12] & & & \\
\hline
\end{tabular}

$>c\{:, 2\}$

$\begin{array}{lllll}\text { ans }= & 0 & & & \\ 1 & & & & \\ 1 & 0 & & & \\ 1 & 0 & 1 & & \\ 1 & 0 & 1 & 0 & \\ 0 & 1 & & & \\ 0 & 0 & & & \\ 1 & 1 & & & \\ 1 & 0 & 1 & 0 & 1 \\ 0 & 0 & 0 & & \\ 0 & 1 & 1 & & \\ 0 & 0 & 1 & & \end{array}$

$>>c\{: 4\}$

ans $=\begin{array}{lllll}0 & 0 & 0 & 0 & 0 \\ 0 & 0 & 0 & 0 & 1 \\ 0 & 0 & 1 & 0 & 0 \\ 0 & 0 & 1 & 1 & 1 \\ 0 & 1 & 0 & 0 & 0 \\ 0 & 0 & 0 & 1 & 1 \\ 0 & 0 & 0 & 1 & 0 \\ 0 & 0 & 1 & 0 & 1 \\ 0 & 1 & 0 & 1 & 1 \\ 0 & 1 & 1 & 1 & 0 \\ 0 & 1 & 1 & 0 & 1 \\ 0 & 1 & 1 & 1 & 1\end{array}$

It can be seen that the first, second (c $\{:, 2\})$, and fourth $(c\{:, 4\})$ columns in the cell $c$ are identical to their respective columns obtained in the earlier example. The third column in the cell $\mathrm{c}$ contains only the first number found in the memory representation 
column in the example; the innovation bit part is omitted. The reason for that is that the innovation bit is not needed to be in the third column in order to create the fourth column. It is faster to convert the third column to its binary representation without considering the innovation bit and then just add the innovation bit when forming the code word. The same Matlab file as being used for the main simulation is used. The only change is that for the example the input sequence is 29 bit stream given in the example. 


\section{Chapter 4: CHANNEL CODEC}

\subsection{Channel Codes}

\subsubsection{Parity Check Codes}

The discussion on channel codes will begin with the introduction of parity check codes. Only the binary code words of information will be considered meaning that any code word consists of only two digits as the elements of the binary alphabet: a 1 and a 0 . Those code words are called binary code words. Parity check codes involve the arithmetic operations of both addition and multiplication performed on binary code words. Since the binary alphabet is used to represent any binary code word, the operations of addition and multiplication performed on binary code words are also called the binary addition and binary multiplication. These operations are performed according to the conventions of the algebraic set $S$ that contains the alphabet's symbols as its elements. The set satisfies the following properties associated with the binary addition:

1. if $a, b$ are binary code words and $a, b \in S$, then $a+b \in S$;

2. if $a, b$, and $c \in S$, then $a+(b+c)=(a+b)+c$;

3. if $a, b \in S$, then $a+b=b+a$;

4. the set $S$ contains a zero element that satisfies the condition: $a+0=a$;

5. every element in the set $\mathrm{S}$, except zero, has its own negative element and their addition yields the zero element. Hence, if $a \in S$, its negative element is - a and $\mathbf{a}+(-\mathbf{a})=0$;

6. the operation, designated with the symbol $\oplus$ is defined as:

$$
\begin{aligned}
& 0 \oplus 0=0 \\
& 0 \oplus 1=1 \\
& 1 \oplus 0=1 \\
& 1 \oplus 1=0
\end{aligned}
$$

and the following ones for the binary multiplication:

1. if $a, b$ are binary code words and $a, b \in S$, then $a \cdot b \in S$;

2. if $a, b$, and $c \in S$, then $a \cdot(b \cdot c)=(a \cdot b) \cdot c$;

3. if $a, b \in S$, then $a \cdot b=b \cdot a$;

4. if $a, b$, and $c \in S$, then $(a+b) \cdot c=a \cdot c+b \cdot c$; 
5. every element in the set has its own 'identity' element ' 1 ', where $\mathbf{a} \cdot 1=\mathbf{a}$;

6. every element in the set, except zero, has its 'inverse', where $a \cdot a^{-1}=1$;

7. the operation is defined as:

$$
\begin{aligned}
& 0 \times 0=0 \\
& 0 \times 1=0 \\
& 1 \times 0=0 \\
& 1 \times 1=1
\end{aligned}
$$

Parity check codes are types of codes that use the sum of all bits present in information, or in a block of information, to detect errors inflicted in the information as a result of channel imperfections. We differentiate two types of parity check codes: the single parity check code and the rectangular code.

In the single parity check code all bits in a codeword are summed up and a resulting bit, the parity bit, is concatenated at the beginning of the code word, as shown in Figure 7. The parity bit could be either a 1, symbolizing that the summation of all the bits in the code word yields an odd result, or a 0 , symbolizing that the summation of all the bits in the code word yields an even result. If the added parity bit is designed to yield an even result, the method is called the even parity method. Similarly, if it yields an odd result, it is called the odd parity method.

Code word: $0 \begin{array}{llllllll}0 & 1 & 0 & 1 & 0 & 1 & 0 & 1 \text {; }\end{array}$

Parity bit: $0+1+0+1+0+1+0+1=0$;

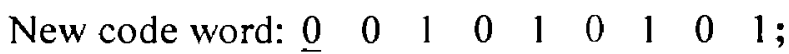

Figure 7: Single Parity Check Code.

In the rectangular code, a rectangle of code words is formed containing I rows and $\mathrm{J}$ columns. Now, for each of 1 rows, a horizontal parity bit is appended at the beginning of each row. Also, for each of J columns, including the very first column representing the horizontal parity bits, a vertical parity bit is appended at the beginning of each column, creating a new block of the $(\mathrm{I}+1) \times(\mathrm{J}+1)$ dimensions.

Let's now take a look at the error detection capabilities of these two codes. The single parity check code can only detect errors if the odd number of bits in a code word is wrongly received. If the number of corrupted bits in a code word is even, the code will not be able to detect it since the parity bit will remain the same. Even though the single 
parity code can detect the odd number of corrupted bits, it cannot correct them since the code has no knowledge where in the code word the corrupted bit(s) is(are) located. On the other hand, unlike the single parity code the rectangular code can correct a single error anywhere in the block (rectangular) of code words since every bit in the $I \times J$ block is uniquely located at the intersection of the horizontal and vertical parity bits.

\begin{tabular}{|c|c|c|c|c|c|c|c|}
\hline \multirow{6}{*}{\multicolumn{2}{|c|}{ 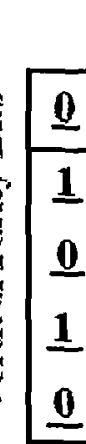 }} & \multicolumn{6}{|c|}{ Holizontal Parity Bits } \\
\hline & & $\underline{0} 1$ & $\underline{\underline{a}}$ & $\underline{0}$ & 1 & & $\underline{0}$ \\
\hline & & 0 & 0 & 1 & & & \\
\hline & & & 0 & 0 & 0 & 1 & 0 \\
\hline تِّ.ّ. & & & 1 & 1 & 0 & 0 & 0 \\
\hline & & & & $\mathbf{0}$ & $\mathbf{0}$ & 0 & 1 \\
\hline
\end{tabular}

Figure 8: Rectangular Parity Code $(\mathrm{l}=4, \mathrm{~J}=6)$.

For example, if in the block shown in Figure 8, the bit in the second row and third column were received as a 1 , then the horizontal parity bit for the second row would be 1 instead of 0 , while the vertical parity bit for the third row would change from 0 to 1 . This way the location of the corrupted bit is easily identified according to the locations of horizontal and vertical parity bits. Rectangular codes are defined by two parameters: $\mathbf{k}$ and $\mathrm{n}$, where $\mathrm{k}=\mathrm{I} \cdot \mathbf{J}$ and $\mathrm{n}=(\mathrm{I}+\mathrm{I}) \cdot(\mathrm{J}+1)$. It is a common practice that rectangular codes are depicted with the $(n, k)$ notation. For the case in Figure 8, we say that the shown rectangular code is of type $(35,24)$.

\subsubsection{Linear Block Codes}

Linear block codes are, as mentioned earlier, characterized by the ( $n, k)$ notation. The purpose of the code is to encode a block of $k$-bit code words into a bigger block of code words where each code word contains $n$ bits. In other words, the encoding procedure assigns to each of the $2^{k}$ possible $k$-bit code words a unique $n$-bit code word chosen from the set of $2^{n}$ possible code words. A block code represents a one to one assignment where $2^{k} k$-bit code words are mapped to $2^{k} n$-bit code words. The only problem here is how to select a subset of $2^{k} n$-bit code words from the set of $2^{n}$ possible $n$-bit code words. To answer this question we first introduce two terms: a vector space and vector subspace. A vector space $V_{n}$ is the set of all $n$-bit code words defined 
over the binary field of two elements: 0 and 1 . A subset $S_{n}$ of the vector space $V_{n}$ is called a subspace if the following two conditions are met:

1. the subset $S_{n}$ contains the all zero vector,

2. if code words $C_{i}, C_{j} \in V_{n}$ then $C_{k} \in V_{n}$. where $C_{k}=C_{i} \oplus C_{j}$.

The second condition means that the sum of any two code words that belong to the vector space $V_{n}$ must also belong to the vector space $V_{n}$. This condition is fundamental for the existence of linear block codes. Once we have defined the vector space and vector subspace, let's now establish the goal that a chosen block code has to achieve. The selected block code should have as large as possible vector space $\mathrm{V}_{\mathrm{n}}$ (containing as many code words as possible in it). Then, we should select code words that will form the subspace $S_{n}$ in the way that the selected code words are as far apart from each other in the subspace as possible. Now, we have to define the distance from two code words in a subspace in order to select those that are as far apart as possible. The Humming distance is the measure of distance between two code words. The Humming distance is defined to be the number of elements in which two code words differ. Why is it so important that two words are as far apart as possible?

Let's assume that on the receiver side a received code word has 2 corrupted bits. If the corrupted received code word is the same as another code word from the subspace, there would be no way that the corrupted bits in the received word are detected as erroneous and possibly corrected. The decoder would simply consider the received code word as a valid code word from the designated subspace set and no correction will be performed. Everything looks O.K. but the problem is that the decoded symbol is not the one that had been transmitted. This is exactly why it is desirable that code words in a subspace have as many different bits among themselves as possible in order to prevent the above situation. However, choosing code words from a subspace that are as far apart as possible is not an easy job by any means.

A lot of researches have been done on the issue of finding code words from a subspace that are as far apart as possible but so far no acceptable algorithm has been developed that would help us select the best set of code words for a subspace. In this project we will follow the recommendation from the B. Sclar's book "Digital Communications", where it is suggested that for a particular subspace to be considered as valid one, it is sufficient that the code words from the subspace satisfy the conditions given by the equations (28a) and (28b). In other words, the subspace contains the all zero element and the sum of any two code words in the subspace is another code word that also belongs to the subspace. 
Let's now consider a block code with $n=7$ and $k=4$, a $(7,4)$ block code. The code has the vector space $V_{7}$ containing $2^{7}=128$ distinct code words, and the vector subspace $S_{4}$ that need to have $2^{4}=16$ code words. We can choose any 16 code words out of 128 possible code words from the vector space as long as they satisfy the conditions (28a) and (28b). Here are, for instance, four code words that satisfy both (28a) and (28b) conditions and can be a part of any $\mathrm{S}_{4}$ subspace:

$$
\begin{array}{lllllll}
0 & 0 & 0 & 0 & 0 & 0 & 0 \\
1 & 0 & 1 & 0 & 1 & 0 & 1 \\
0 & 1 & 0 & 1 & 0 & 1 & 0 \\
1 & 1 & 1 & 1 & 1 & 1 & 1
\end{array}
$$

\begin{tabular}{rrrrr}
1010101 & 0101010 & 1111111 & 1111111 \\
+0101010 & +1111111 & +1010101 & +0101010 \\
\hline 1111111 & 1010101 & 0101010 & 1010101
\end{tabular}

We see that the selected four code words contain the all zero code word and that the sum of any two code words, among those four code words, produces a code word that is one of those four code words. Addition between the all zero code word with the remaining three code words is omitted as obvious. One of the ways to encode a message with a $(7,4)$ block code is to use a look up implementation and for each message look up in the table an associate code word. But would that work if we, for example, had $k=92$, as it is the case in the real world. Certainly not, since we would need a table with $4.95 \cdot 10^{27}$ entrances. Time wise, it would be totally unacceptable, or to be more realistic, it would be impossible.

\subsubsection{Linear Block Code Encoding}

Luckily, it is possible to obtain a set of $n$-bit code words that will generate all $2^{k}$ vectors of the subspace. In other words, any of $2^{\mathrm{k}}$ possible $\mathrm{n}$-bit code words can be generated by using the following matrix equation:

$$
\overline{\mathrm{U}}=\overline{\mathrm{m}} \cdot \overline{\mathrm{G}}=\mathrm{m}_{1} \cdot \overline{\mathrm{G}}_{1}+\mathrm{m}_{2} \cdot \overline{\mathrm{G}}_{2}+\cdots+\mathrm{m}_{\mathrm{k}} \cdot \overline{\mathrm{G}}_{\mathrm{k}} \cdot
$$

where $m=\left[m_{1} m_{2} \cdots m_{k}\right]$ is a vector that contains all message symbols that are to be transmitted, and $\bar{G}$ is a 'generator matrix' with the dimensions $(k \times n)$ : 


$$
\overline{\mathrm{G}}=\left[\begin{array}{c}
\overline{\mathrm{G}}_{1} \\
\overline{\mathrm{G}}_{2} \\
\vdots \\
\overline{\mathrm{G}}_{\mathrm{k}}
\end{array}\right]=\left[\begin{array}{cccc}
\mathrm{g}_{11} & \mathrm{~g}_{12} & \cdots & \mathrm{g}_{1 \mathrm{n}} \\
\mathrm{g}_{21} & \mathrm{~g}_{22} & \cdots & \mathrm{g}_{2 \mathrm{n}} \\
\vdots & & & \\
\mathrm{g}_{\mathrm{k} 1} & \mathrm{~g}_{\mathrm{k} 2} & \cdots & \mathrm{g}_{\mathrm{kn}}
\end{array}\right]
$$

In (30) the vectors $\overline{\mathrm{G}}_{1}, \overline{\mathrm{G}}_{2}, \ldots, \overline{\mathrm{G}}_{\mathrm{k}}$ are linearly independent vectors that can generate all code words. If the generated code words contain the original messages that are parts of the code words, then we say that the code is 'systematic' and the generator matrix is of the following form:

$$
\overline{\mathrm{G}}=\left[\begin{array}{lll}
\overline{\mathrm{P}} & \mid & \overline{\mathrm{I}}_{k}
\end{array}\right]
$$

where the matrix $\bar{P}$ is a parity matrix of the $(k \times(n-k))$ dimensions, while $\bar{I}_{k}$ is an identity matrix, the dimensions $(\mathrm{k} \times \mathrm{k})$, with ones on the main diagonal and zeros elsewhere. This simplifies the process of encoding significantly since now we only have to find a matrix $\bar{P}$ in order to obtain a generator matrix $\bar{G}$. Once the generator matrix $\bar{G}$ is known we can easily encode any message by using the equation (29).

To sum up the process of encoding of a message with a $(n, k)$ block code: we first create a subspace of $2^{k} n$-bit code words. Among those code words we select $k$ of them in order to generate a generator matrix $\bar{G}$, in such a way that our generator matrix is created from a parity matrix $\overline{\mathrm{P}}$ and an identity matrix $\overrightarrow{\mathbf{I}}_{\mathrm{k}}$. Now, once we obtain the generator matrix we encode any message symbol by simply applying the equation (29). The process is illustrated in a simplified flow chart shown below:

$$
\overline{\mathrm{m}} \rightarrow \overline{\mathrm{G}} \rightarrow \overline{\mathrm{U}}
$$

\subsubsection{Linear Block Code Decoding}

When it comes to decoding of a received code word, one should think that the process of decoding would just be an opposite process from the process of encoding. By looking at the linear block code encoding flow chart in (32), it would be natural to think that by multiplying the received code word with the inverse generator matrix, we would be able to obtain the transmitted message. The idea sounds O.K. but unfortunately it does not work since an inverse generator matrix $\bar{G}^{-1}$ does not exist in the sense that it would help us recover the encoded message symbol. To overcome this problem we will keep the 
initial decoding idea presented earlier but instead of an inverse generator matrix we will introduce a parity check matrix $\bar{H}$ that indirectly enables us to decode the received code word, where the parity check matrix $\bar{H}$ with its dimensions $((n-k) \times n)$ is defined as:

$$
\overline{\mathrm{H}}=\left[\begin{array}{lll}
\overline{\mathbf{I}}_{\mathrm{n}-\mathrm{k}} & \mid & \overline{\mathrm{P}}^{\mathrm{T}}
\end{array}\right] .
$$

We know that a received code word, encoded at the transiver side, is an n-bit vector with dimensions $(1 \times \mathrm{n})$ and that the matrix multiplication between the received code word and the parity check matrix $\bar{H}$, with dimensions $((n-k) \times n)$, is mathematically impossible. So, how do we then decode the received code word?

If we take a careful look at the matrix $\overline{\mathrm{H}}$ we can notice that if we transpose the matrix we get the dimensions that allow us to perform the matrix multiplication between the transposed parity check matrix and the received code word. The transposed parity check matrix is defines as:

$$
\overline{\mathrm{H}}^{\mathrm{T}}=\left[\begin{array}{c}
\overline{\mathrm{I}}_{\mathrm{n}-\mathrm{k}} \\
--- \\
\overline{\mathrm{P}}
\end{array}\right] \text {. }
$$

Can we consider the result of the matrix multiplication between the transposed parity check matrix and the received code word to be the recovered original message symbol? No, at least not yet. With the matrix multiplication between the transposed parity check matrix and the received code word we obtain a $(I \times n)$ vector $\vec{S}$ called a syndrome:

$$
\overline{\mathrm{S}}=\overline{\mathrm{r}} \cdot \overline{\mathrm{H}}^{\mathrm{T}} \text {, }
$$

where the vector $\bar{r}$ is a received code word. The purpose of the syndrome vector is to determine if the received code word has been corrupted during the transmission. Here is how the syndrome vector $\overline{\mathrm{S}}$ achieves it. We will start with the assumption that the received code word has been corrupted during the transmission and that it is defined as:

$$
\bar{r}=\bar{U}+\bar{e},
$$

where the vector $\bar{U}$ is one of $2^{k}$-bit code words from the subspace, while the vector $\overline{\mathrm{e}}$ is the error vector introduced by the channel. From the equation (35) we obtain the syndrome $\bar{S}$ of the received code word $\bar{r}$. If the syndrome $\bar{S}$ is an all zero vector, then 
the received code word has not been corrupted by the cliannel. Conversely, if the syndrome $\bar{S}$ is not an all zero vector, then the received code word has been corrupted by the channel and the decoder has to make an effort to correct the corrupted code word. Before we get to that point, let's first rearrange the equation (35) by replacing the vector $\bar{r}$ with the value from the equation (36):

$$
\begin{aligned}
& \overline{\mathrm{S}}=(\overline{\mathrm{U}}+\overline{\mathrm{e}}) \cdot \overline{\mathrm{H}}^{\mathrm{T}}=\overline{\mathrm{U}} \cdot \overline{\mathrm{H}}^{\mathrm{T}}+\overline{\mathrm{e}} \cdot \overline{\mathrm{H}}^{\mathrm{T}}=\overline{\mathrm{e}} \cdot \overline{\mathrm{H}}^{\mathrm{T}}, \\
& \text { since } \quad \overline{\mathrm{U}} \cdot \overline{\mathrm{H}}^{\mathrm{T}}=\overline{\mathrm{m}} \cdot \overline{\mathrm{G}} \cdot \overline{\mathrm{H}}^{\mathrm{T}}=\overline{0} .
\end{aligned}
$$

The equality $\overline{\mathrm{G}} \cdot \overline{\mathrm{H}}^{\mathrm{T}}=\overline{0}$ used in (37b) can easily be proven from the equations (31) and (34). The equations (37a) and (37b) tell us that the syndrome vector $\overline{\mathrm{S}}$ has a one to one correspondence between the error vector $\bar{e}$ and the corrupted code word $\bar{r}$. This is a very powerful observation that enables us not only to detect the error but also to correct it.

Let's consider all $2^{n}$ possible n-bit code words that can be received at the receiver. Recall that the number of valid $n$-bit code words belonging to the subspace is $2^{k}$, and that the rest $2^{n}-2^{k} n$-bit code words represent all possible combinations of the corrupted valid code words having the $w$ number of corrupted bits, where $w=1,2, \ldots, n$. If we put what has just been said into a two dimensional array, we will end up with a two

\begin{tabular}{|c|c|c|c|c|c|}
\hline$\overline{\mathbf{U}}_{1}$ & $\overline{\mathbf{U}}_{2}$ & ... & $\overline{\mathbf{U}}_{\mathbf{i}}$ & $\ldots$ & $\overline{\mathbf{U}}_{\mathbf{2}^{\mathfrak{4}}}$ \\
\hline$\overline{\mathbf{e}}_{2}$ & $\overline{\mathbf{U}}_{2}+\overline{\mathbf{e}}_{2}$ & $\ldots$ & $\overline{\mathbf{U}}_{\mathbf{i}}+\overline{\mathbf{e}}_{\mathbf{2}}$ & $\ldots$ & $\overline{\mathrm{U}}_{2^{\mathrm{k}}}+\overline{\mathrm{e}}_{\mathbf{2}}$ \\
\hline$\overline{\mathbf{e}}_{\mathbf{3}}$ & $\overline{\mathbf{U}}_{2}+\overline{\mathbf{e}}_{3}$ & $\cdots$ & $\overline{\mathbf{U}}_{\mathbf{i}}+\overline{\mathbf{e}}_{\mathbf{3}}$ & $\ldots$ & $\overline{\mathrm{U}}_{2^{k}}+\overline{\mathrm{e}}_{\mathbf{3}}$ \\
\hline $\begin{array}{c}\vdots \\
\mathbf{e}_{\mathbf{j}}\end{array}$ & $\begin{array}{c}\vdots \\
\overline{\mathbf{U}}_{2}+\overline{\mathbf{e}}_{\mathbf{j}}\end{array}$ & $\ldots$ & $\begin{array}{c}\vdots \\
\overline{\mathbf{U}}_{\mathbf{i}}+\overline{\mathbf{e}}_{\mathbf{j}}\end{array}$ & $\ldots$ & $\overline{\mathbf{U}}_{2^{\mathrm{k}}}{ }^{\vdots} \overline{\mathbf{e}}_{\mathbf{j}}$ \\
\hline$n^{n-k}$ & $\begin{array}{c}\vdots \\
\overline{\mathbf{U}}_{2}+\overline{\mathbf{e}}_{2^{n-k}}\end{array}$ & $\ldots$ & $\overline{\mathbf{U}}_{\mathbf{i}}+\overline{\mathbf{e}}_{\mathbf{2}^{n-k}}$ & $\ldots$ & $\bar{U}_{2^{k}}+\bar{e}_{2^{n-k}}$ \\
\hline
\end{tabular}
dimensional array as shown in Figure 9.

Figure 9: Decoding Table for Linear Block Codes.

The decoding table for linear block codes must satisfy the following conditions:

1. the first row contains $2^{k}$ valid code words; 
2. the first column, starting from the second element, contains correctable error patterns. The first element in the first column contains the first valid code word from the subspace which is an all zero code word;

3. the rest of the array is filled in such a way that each element in the array is obtained as the sum of the first element of that column (valid code word) and the first element in that row (error pattern);

At this point we have all necessary information in place to detect and correct errors during a transmission, except one; what error patterns are going to be considered detectable and correctable? To determine that, we have to recall the definition of the Humming distance which states that the Humming distance is the number of elements in which two code words differ. Another interesting term here is the Humming weight of a code word that defines the number of non-zero elements in a code word (e.g. for the code word: 1001101 , the Humming weight of the code word is: 4). Both the Humming distance (directly) and the Humming weight (indirectly) are tied to the code's detection and correction capabilities through the following equations:

$$
\begin{array}{r}
\mathrm{e}=\mathrm{d}_{\min }-\mathrm{l}, \\
\mathrm{t}=\left\lfloor\frac{\mathrm{e}}{2}\right\rfloor,
\end{array}
$$

where $\mathrm{d}_{\text {min }}$ is the minimum Humming distance of the code, $\mathrm{e}$ is defined as the error detecting capability of the code, while $t$ is the error correcting capability of the code. At this point we may notice that in order to obtain the error detecting and correcting capabilities we have to calculate the Huffman distance between any two code words in the subspace in order to find the minimum Humming distance of the code. It is easy to realize that it would take a long time, especially for the codes with the large $k$ (e.g. for a $(127,92)$ code where $k=92$, the subspace contains $5 \times 10^{27}$ code words). However, as we have discussed earlier, we know that the sum of any two code words in the subspace yields another code word from the subspace. Thus, the Humming distance between any two code words in the subspace is in fact the Humming weight of the resulting code word. So, instead of calculating the Humming distance for any pair of code words in the subspace, we simply just calculate the Humming weight of the code word obtained by their addition, which theoretically could be any code word in the subspace. It is now easy to conclude that the smallest Humming weight of the code is in fact the minimum Humming distance of the code. 
To sum up the decoding procedure for the systematic block codes: upon the arrival of a code word, we first calculate the syndrome of the received word using the equation (35), then we locate the error pattern in the table that gives a one to one correspondence between syndromes and error patterns. Once the error pattern is identified the received code word is binary added with the identified error pattern and the original message symbol is obtained. We will demonstrate the encoding and decoding procedures with an example that will provide a comprehensive overview of these two procedures. In the example we will use a $(5,3)$ code to encode and decode 8 message symbols.

\section{EXAMPLE (Linear Block Codes):}

a) Find all the code words produced by a $(5,3)$ encoder;

b) Find a combination of error patterns that a $(5,3)$ decoder uses to detect and correct received code words that are errant;

c) Decode the following input sequence to the decoder: 101001110110000 ;

\section{SOLUTION:}

a) $n=5, k=3$. The vector space $V_{5}$ contains $2^{5}=32$ possible 5-bit code words. The subspace $S_{3}$ contains $2^{3}=8$ code words. The number of all possible 3-bit message symbols is $2^{3}=8$. The message symbols are: 000,001,010,011,100,101,110, and 111. One possible combination of 5-bit code words that make up the $S_{3}$ subsystem could be:

$$
\begin{array}{lllll}
0 & 0 & 0 & 0 & 0 \\
1 & 0 & 1 & 0 & 1 \\
0 & 1 & 0 & 1 & 0 \\
1 & 1 & 1 & 1 & 1 \\
1 & 1 & 0 & 0 & 1 \\
0 & 1 & 1 & 0 & 0 \\
1 & 0 & 0 & 1 & 1 \\
0 & 0 & 1 & 1 & 0
\end{array}
$$

The shown subspace satisfies the conditions (28a) and (28b); it contains an all zero vector, and the sum of any two code words yields a code word from the subspace. However, we need to choose only three 5-bit code words from the subspace to obtain all 8 possible code words. In other words we need to build the generator matrix $\bar{G}$ of the dimensions $(k \times n)$ that satisfies the condition from the equation (31): 


$$
\begin{aligned}
\bar{G} & =\left[\begin{array}{lllll}
0 & 1 & 1 & 0 & 0 \\
0 & 1 & 0 & 1 & 0 \\
1 & 1 & 0 & 0 & 1
\end{array}\right] \\
\text { where: } & \bar{P}=\left[\begin{array}{ll}
0 & 1 \\
0 & 1 \\
1 & 1
\end{array}\right] \\
& \text { and } \quad \bar{I}_{3}=\left[\begin{array}{lll}
1 & 0 & 0 \\
0 & 1 & 0 \\
0 & 0 & 1
\end{array}\right]
\end{aligned}
$$

\begin{tabular}{|c|c|c|}
\hline MESSAGE: & CODE WORDS: & HUMMING WEIGHT: \\
\hline 000 & 00000 & 0 \\
\hline 001 & 11001 & 3 \\
\hline 010 & 01010 & 2 \\
\hline 011 & 10011 & 3 \\
\hline 100 & 01100 & 2 \\
\hline 101 & 10101 & 3 \\
\hline 110 & 00110 & 2 \\
\hline 111 & 11111 & 5 \\
\hline
\end{tabular}

Now, by using the equation (3) we can get all 8 code words. They are listed below:

b) $d_{\min }=2$, so we can detect all 1-bit errors in the received code words, and correct $2^{n}-2^{k}-1=3$ error patterns. We will choose that our error patterns are:

$$
\bar{e}=\left[\begin{array}{lllll}
0 & 0 & 1 & 0 & 0 \\
1 & 0 & 0 & 0 & 0 \\
0 & 0 & 0 & 0 & 1
\end{array}\right]
$$

c) First, we rearrange the input sequence into 5-bit received code words:

$$
\begin{aligned}
& \bar{r}_{1}=\left[\begin{array}{lllll}
1 & 0 & 1 & 0 & 0
\end{array}\right] \\
& \bar{r}_{2}=\left[\begin{array}{lllll}
1 & 1 & 1 & 0 & 1
\end{array}\right] \\
& \bar{r}_{3}=\left[\begin{array}{lllll}
1 & 0 & 0 & 0 & 0
\end{array}\right]
\end{aligned}
$$

Next, we find transposed parity check matrix $\bar{H}^{T}$ with the $((n-k) \times n)$ dimensions using the equation (34): 


$$
\vec{H}^{T}=\left[\begin{array}{ll}
1 & 0 \\
0 & 1 \\
0 & 1 \\
0 & 1 \\
1 & 1
\end{array}\right]
$$

where: $\quad \bar{I}_{2}=\left[\begin{array}{ll}1 & 0 \\ 0 & 1\end{array}\right] \quad$ and $\quad \bar{P}=\left[\begin{array}{ll}0 & 1 \\ 0 & 1 \\ 1 & 1\end{array}\right]$

Now, we calculate the syndrome matrix from the equation (37a):

$$
\bar{S}=\bar{e} \cdot \bar{H}^{T}=\left[\begin{array}{ll}
0 & 1 \\
1 & 0 \\
1 & 1
\end{array}\right]
$$

Then, we form a one to one correspondence between the error patterns $\bar{e}$ and the syndrome $\bar{S}$ :

$$
\left[\begin{array}{lllll}
0 & 0 & 1 & 0 & 0 \\
1 & 0 & 0 & 0 & 0 \\
0 & 0 & 0 & 0 & 1
\end{array}\right] \Leftrightarrow\left[\begin{array}{ll}
0 & 1 \\
1 & 0 \\
1 & 1
\end{array}\right]
$$

The following thing is to calculate the individual syndromes for the each received code word as per equation (35):

$$
\bar{S}_{I}=\left[\begin{array}{ll}
1 & 1
\end{array}\right] \quad \bar{S}_{2}=\left[\begin{array}{ll}
0 & 1
\end{array}\right] \quad \bar{S}_{3}=\left[\begin{array}{ll}
1 & 0
\end{array}\right]
$$

Using the obtained individual syndromes we find their corresponding error patters from $\bar{e}$ :

$$
\begin{aligned}
& \bar{e}_{1}=\left[\begin{array}{lllll}
0 & 0 & 0 & 0 & 1
\end{array}\right] \\
& \bar{e}_{2}=\left[\begin{array}{lllll}
0 & 0 & 1 & 0 & 0
\end{array}\right] \\
& \bar{e}_{3}=\left[\begin{array}{lllll}
1 & 0 & 0 & 0 & 0
\end{array}\right]
\end{aligned}
$$

To obtain the correct code words we perform the following binary addition:

$$
\bar{U}=\bar{r}+\bar{e}
$$


That yields:

$$
\begin{aligned}
& \bar{U}_{1}=\left[\begin{array}{lllll}
1 & 0 & 1 & 0 & 1
\end{array}\right] \\
& \bar{U}_{2}=\left[\begin{array}{lllll}
1 & 1 & 0 & 0 & 1
\end{array}\right] \\
& \bar{U}_{3}=\left[\begin{array}{lllll}
0 & 0 & 0 & 0 & 0
\end{array}\right]
\end{aligned}
$$

From here it is easy to determine which message symbols have been transmitted. By using the table from the part a) we can easily find the corresponding message symbols:

$$
\bar{m}_{1}=\left[\begin{array}{lll}
1 & 0 & 1
\end{array}\right] \quad \bar{m}_{2}=\left[\begin{array}{lll}
0 & 0 & 1
\end{array}\right] \quad \bar{m}_{3}=\left[\begin{array}{lll}
0 & 0 & 0
\end{array}\right]
$$

\subsubsection{Matlab Implementation of Linear Block Codes}

The following Matlab files simulate the linear block code implementation:

The Matlab file "Generate_Matrices.m" that creates the $(12,8)$ linear block code that is used for the project simulation. The file also creates the following matrices needed for the encoding and decoding procedures: $\overline{\mathrm{I}}_{\mathrm{k}}, \overline{\mathrm{P}}, \overline{\mathrm{G}}, \overline{\mathrm{H}}^{\mathrm{T}}$, $\overline{\mathrm{e}}$, and $\overline{\mathrm{S}}$. The "LBC_Encode.m" and "LBC_Decode.m" Matlab files that perform the encoding and decoding procedures implemented in an exact fashion as presented in the discussion and example earlier.

To conclude the discussion on linear block codes, let's take a look at the printout of the Matlab code that works out the example above (the code for "LBC_Example" file is provided in the Appendix $B$ of this report):

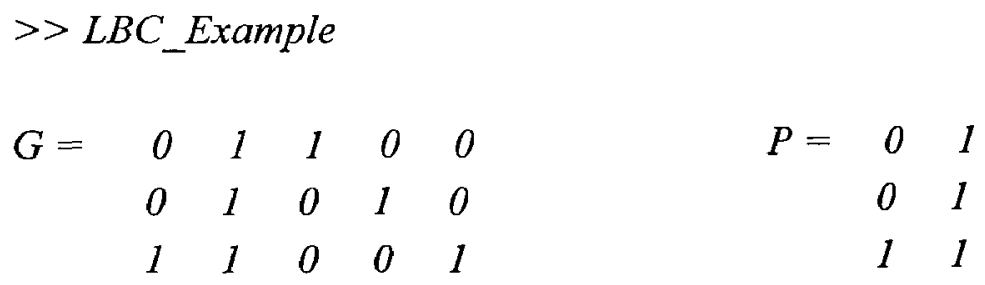

$\begin{array}{ll}\text { message }\{1\}=000 & U\{1\}=00000 \\ \text { message }\{2\}=001 & U\{2\}=11001 \\ \text { message }\{3\}=010 & U\{3\}=01010 \\ \text { message }\{4\}=011 & U\{4\}=10011 \\ \text { message }\{5\}=100 & U\{5\}=01100 \\ \text { message }\{6\}=101 & U\{6\}=10101\end{array}$




$$
\begin{array}{ll}
\text { message }\{7\}=110 & U\{7\}=00110 \\
\text { message }\{8\}=111 & U\{8\}=11111
\end{array}
$$

received corrupted sequence $=101001110110000$

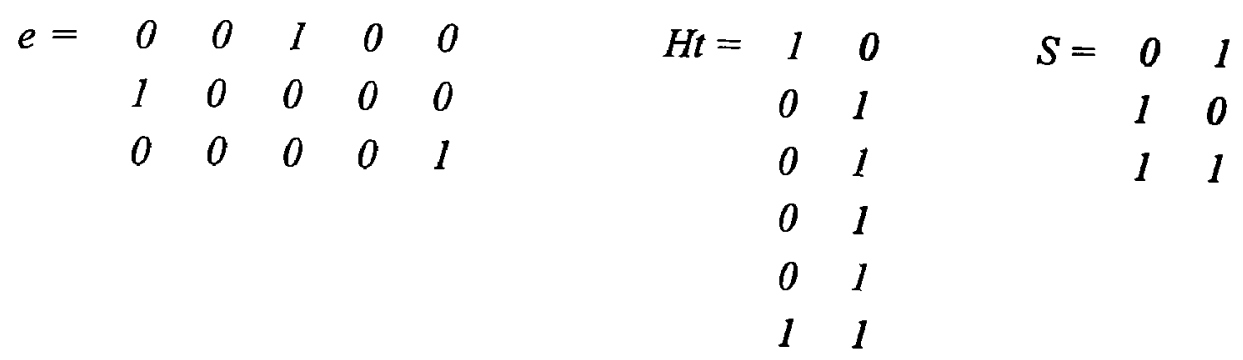

decoded_message_symbols $=101001000$

We see that in both cases, the example and the Matlab code, we get the same results obtaining the same decoded message symbols: 101001000 as expected.

\subsection{Convolutional Codes}

Convolutional codes are generated by passing a specified number of bits, or a single bit itself, through a linear finite state register. The linear finite state register is also commonly called a convolutional encoder. Convolutional codes are usually specified by three parameters: $\mathrm{n}, \mathrm{k}$, and $\mathrm{m}$, where $\mathrm{k}$ is a number of bits in a codeword coming in the encoder (input sequence), $\mathrm{n}$ is a number of bits in a codeword going out of the encoder (output codeword), and $m$ is a number of stages of memory registers. From the definition of the index $\mathrm{m}$ we note an important characteristic of Convolutional codes; convolutional encoders contain memory. The encoder in Figure 9 is classified as a $(2,1,4)$ convolutional encoder due to the following reasons: the output of the encoder contains a 2-bit codeword, the input to the encoder contains a 1-bit input sequence that is shifted through 4 stages of the memory register (encoder).

It is assumed that each bit in the input sequence (in the case in Figure 9 only one bit makes the input sequence) is equally likely to be either 1 or 0 and is independent from any other bit in the sequence. The mutual bit independency is exactly a main reason why we are able to perform the channel coding operation before the data is transmitted out. For example, if during a transmission one or more bits of information are incorrectly received; from the receiver's prospective what can be done to correct those corrupted bits? 


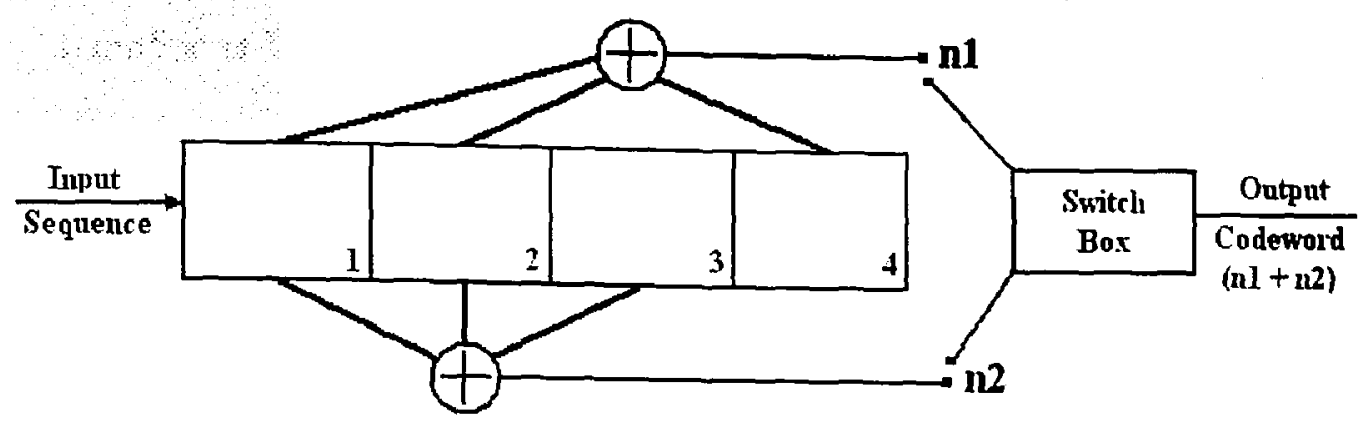

Figure 10: $(2,1,4)$ Convolutional Encoder.

First of all, there would be no way we could determine that the transmission has caused a corruption of a bit, or stream of bits, unless we have a previous knowledge of the data that had been sent out. Secondly, the concepts mentioned in the previous sentence pretty much contradict each other; if we know in advance what is to be transmitted, then why do we need to transmit it at all? This is where convolutional codes come into the picture by adding a previously determined number of redundant bits that carry the information about the order and structure of bits that the data is formed of. Convolutional codes help control the order and structure of the data bits at the receiver side where some bits might be received in error. In general, it is the function of the encoder to introduce redundant bits in the transmitted information sequence which then can be used at the receiver side to overcome the effects of noise and interference encountered during the transmission of the signal through the channel. The amount of redundancy introduced during the encoding procedure is measured by the ratio $n$ over $k$.

Once the convolutional code indexes ( $\mathrm{n}, \mathrm{k}$, and $\mathrm{m}$ ) are determined (the responsibility of the designer), the convolutional encoder is easy to implement. First, $m$ boxes representing the $m$ memory register stages are drawn, then $n$ modulo- 2 adders are placed, and finally the memory register stages are connected to the adders using the generator polynomial vector. The generator polynomial vector $\overrightarrow{\mathrm{g}}$ determines a way in which the stages of the memory register are going to be connected to the modulo-2 adders. The generator polynomials, as the elements of the generator polynomial vector (also called connection vectors), give the convolutional code its unique error protection quality. There are many choices for a selection of generator polynomials for an $m$ order code. However, they all do not result in output codeword sequences that have good error protection properties. Those generator polynomials that do provide good error protection properties are usually obtained by computer simulations. The generator polynomials used for the simulation of a convolutional code in this project are taken from Peterson and Weldon's book "Error Correcting Codes" and they are presented in Table 1: 


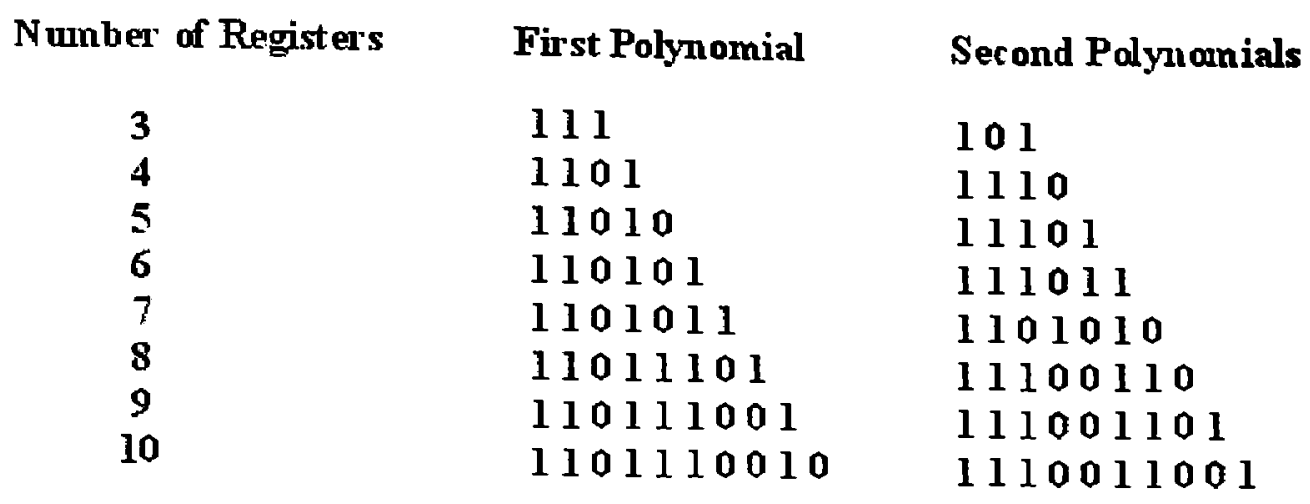

Table 1: Generator Polynomials for Convolutional Codes.

From Table 1 we conclude that we must have the same number of generator polynomials as we have output bits from the encoder. Also, each generator polynomial would contain $m$ elements that can be represented by a 1 or a 0 , where 1 represents a connection and 0 the absence of the connection between an adder and a memory stage. In the case of the $(2,1,4)$ encoder shown in Figure 10, we have two output bits $(n=2)$ and four stages of a register $(m=4)$. Thus, we would need two generator polynomials, each of whom would have 4 elements.

\subsubsection{Convolutional Code Encoding}

Several methods are used to represent the process of convolutional encoding. The most popular ones are: impulse response of the encoder, polynomial representation, state representation and the state diagram, and the trellis diagram. Each of these methods will be briefly discussed here in this chapter while the impulse response and the polynomial representation methods will be implemented in the Matlab simulation.

Let's now go back to our $(2,1,4)$ convolutional encoder from Figure 10. At each input time, one bit from the input sequence is shifted into the leftmost stage (box 1) of the encoder and the bits in the other stages of the encoder (boxes 2, 3, and 4) are shifted one position to the right. The switch box at the output samples the outputs from the adders, interlaces them, and forms an output codeword $\left(n_{1} \& n_{2}\right)$. The sampling process keeps on repeating until no more bits are available at the input of the encoder. The connections between the modulo- 2 adders and the memory stages of the $(2,1,4)$ encoder are given by the following two generator polynomials:

$$
\mathrm{g} 1=\left[\begin{array}{llll}
1 & 1 & 0 & 1
\end{array}\right] \text { and } \mathrm{g} 2=\left[\begin{array}{llll}
1 & 1 & 1 & 0
\end{array}\right]
$$




\subsubsection{Impulse Response of the Convolutional Encoder}

After having described the structure of the convolutional encoder in Figure 10, we will now take a look at the functionality of the encoder and how it responses to a single input bit 1 that moves throughout the register's stages from the left to the right. This is called the impulse response of the encoder and creates the so-called a walking one effect that simulates the impulse excitation of the encoder. At the certain time t only one stage of the register (encoder) contains a 1 while the rest of the stages are populated with zeros. The corresponding outputs at $\mathrm{n}_{1}$ and $\mathrm{n}_{2}$ are obtained and saved in two separate vectors. At the time $t+1$, the 1 moves into the next right stage of the register while the previously occupied stage is populated with a 0 . The output bits $n_{1}$ and $n_{2}$ are again obtained and added to the existing values in the vectors. The process is repeated until the 1 is walked through all the stages of the register:

\begin{tabular}{|c|c|c|}
\hline Time & Register Contents & Output bits: $n_{1} n_{2}$ \\
\hline 1 & 1000 & 11 \\
\hline 2 & 0100 & 1 \\
\hline 3 & 0010 & 0 \\
\hline 4 & $\begin{array}{lllllllll}0 & 0 & 0 & 1\end{array}$ & 1 \\
\hline
\end{tabular}

Table 2: Impulse Response of the $(2,1,4)$ Encoder.

From Table 2 we see that for each time $t$ corresponding outputs from $n_{1}$ and $n_{2}$ are sampled and stored creating vectors: $n_{1}$ and $n_{2}$. The impulse response of the encoder is formed by alternately taking bits from vectors $n_{1}$ and $n_{2}$ at times $t=1,2,3$, and 4 :

Impulse Response of the Encoder $=11110110$.

Now when we know the impulse response of the encoder, we can easily find the response of the encoder to any type of input. All we have to do is to apply the principle of the superposition, or linear addition of the time shifted impulses. For instance if we want to obtain the codeword for the input sequence $\left[\begin{array}{llll}1 & 0 & 1 & 1\end{array}\right]$, all we have to do is that for each 1 in the input sequence we assume that the encoder produces the impulse response at the output, and for each 0 in the input sequence the encoder produces a zero response at the output. At each time $t$ we have two outputs of the encoder: $n_{1}$ and $n_{2}$, and at the time $t+$ 1 the output response of the encoder will be shifted two bits to the right compared to the output response at time $t$ : 
$\begin{array}{cc}{[\mathrm{t}]} & \text { [Input] } \\ 1 & 1 \\ 2 & 0 \\ 3 & 1 \\ 4 & 1\end{array}$

The resulting codeword:

\section{[Output]}

11110110

000000000

1111110110

11110110 (impulse response)

(zero response)

(impulse response)

(impulse response)

\section{$11111010101110 \quad$ (input response)}

\subsubsection{Polynomial Representation of Convolutional Encoding}

Another method that a convolutional encoder can be represented with is the polynomial representation. In the polynomial representation each generator polynomial is represented by a real polynomial. Since in the binary world addition and subtraction give the same results, the sign of each term in the polynomial representation is of no interest to us. As a convention, we will assume that each polynomial factor contains a positive sign. The time will be represented by the degree of each polynomial factor. An input bit that gets into the encoder at time $t=1$ will be multiplied by the polynomial factor on the $t-1$ degree, which means if a 1 is a first input bit entering the encoder it would be assigned the $\mathrm{X}^{0}$ polynomial factor. If we now go back to the encoder in Figure 10, then the generator polynomials in the polynomial representation will be declared as:

$$
\begin{aligned}
& g_{1}(X)=1 \cdot X^{0}+1 \cdot X^{1}+0 \cdot X^{2}+1 \cdot X^{3}=1+X+X^{3} \\
& g_{2}(X)=1 \cdot X^{0}+1 \cdot X^{1}+1 \cdot X^{2}+0 \cdot X^{3}=1+X+X^{2}
\end{aligned}
$$

If we want to encode the input sequence $m=\left[\begin{array}{llll}1 & 0 & 1 & 1\end{array}\right]$ that we have used in the previous section, then that input sequence in the polynomial representation would look like:

$$
m(X)=1 \cdot X^{0}+0 \cdot X^{1}+1 \cdot X^{2}+1 \cdot X^{3}=1+X^{2}+X^{3}
$$

The outputs of the adders $\left(\mathrm{n}_{1}\right.$ and $\mathrm{n}_{2}$ ) are found by multiplying both generator polynomials, $g_{1}(X)$ and $g_{2}(X)$ individually with the input polynomial, $m(X)$ :

$$
\begin{aligned}
\mathrm{n}_{1}(X)=\mathrm{m}(X) \cdot \mathrm{g}_{1}(X) & =1 \cdot X^{0}+1 \cdot X^{1}+1 \cdot X^{2}+3 \cdot X^{3}+1 \cdot X^{4}+1 \cdot X^{5}+1 \cdot X^{6} \\
& =1+X+X^{2}+3 \cdot X^{3}+X^{4}+X^{5}+X^{6}
\end{aligned}
$$

In the result for $\mathrm{n}_{1}$ from above, we can notice that the polynomial factor $\mathrm{X}^{3}$ has the prefix 3 as a result of the multiplication. However, we know that in a binary system we can have only two numbers a 0 and a 1 . The number 3 in binary, is in fact, $1+1+1$, and is equal to 1 applying the method of binary addition. Now we have: 


$$
\mathrm{n}_{1}(X)=1+X+X^{2}+X^{3}+X^{4}+X^{5}+X^{6}
$$

which is basically equal to $n_{1}=\left[\begin{array}{lllllll}1 & 1 & 1 & 1 & 1 & 1 & 1\end{array}\right]$. Similarly, we get the following result for the second output $\mathrm{n}_{2}$ :

$$
\begin{aligned}
\mathrm{n}_{2}(X)=m(X) \cdot g_{2}(X) & =1 \cdot X^{0}+1 \cdot X^{1}+2 \cdot X^{2}+2 \cdot X^{3}+2 \cdot X^{4}+1 \cdot X^{5}+0 \cdot X^{6} \\
& =1+X+2 \cdot X^{2}+2 \cdot X^{3}+2 \cdot X^{4}+X^{5} \\
& =1+X+X^{5}
\end{aligned}
$$

Since $2=1+1=0$ in the binary system, the polynomial factors $\mathrm{X}^{2}, \mathrm{X}^{3}$, and $\mathrm{X}^{4}$ will diminish. This is equal to the vector $n_{2}$ where $n_{2}=\left[\begin{array}{lllllll}1 & 1 & 0 & 0 & 0 & 1 & 0\end{array}\right]$. The final codeword is obtained by alternately taking one bit from the vectors $n_{1}$ and $n_{2}$ :

\section{Codeword: 11111010101110.}

The following two methods of the convolutional encoder representation: the state diagram and the trellis diagram will not be discussed in details. Rather, they will be introduced in such a manner and quantity that a reader can follow the rest of the report and be able to understand the decoding techniques based on the state and trellis representations of a convolutional code.

\subsubsection{State Diagram}

The state diagram represents the possible contents (states) of the rightmost $\mathrm{m}-1$

\begin{tabular}{|c|c|c|c|c|}
\hline Current State & \multicolumn{2}{|c|}{ NextState } & \multicolumn{2}{|c|}{ Output } \\
\hline -.---. & & 1 & 0 & 1 \\
\hline$-\cdots-$ & $\ldots$ & $\ldots$ & $n 1 \mathrm{n2}$ & $n 1 n 2$ \\
\hline 000 & 000 & 100 & 00 & 11 \\
\hline 001 & 000 & 100 & 10 & \\
\hline 010 & 001 & 101 & 01 & 10 \\
\hline 011 & 001 & 101 & 11 & 0 \\
\hline 100 & 010 & 110 & & 0 0 \\
\hline 101 & 010 & 110 & 01 & 10 \\
\hline 110 & 011 & 111 & & 01 \\
\hline 111 & 011 & 111 & 00 & 11 \\
\hline
\end{tabular}
register stages ( $m$ is the number of the memory register stages), and the transitions between states as a result of the arrival of a new input bit to the leftmost register stage. Table 3 provides the state diagram for the convolutional encoder shown in Figure 10.

Table 3: State Diagram of the $(2,1,4)$ Convolutional Encoder. 
Since the encoder in Figure 10 has 4 stages of the memory register, its last 3 stages will create the states of the encoder. The last 3 stages can generate $2^{3}=8$ possible states. The next state that the current state moves to every time a new bit enters the encoder depends on the fact if the new bit is either a 0 or a 1 . To sum up. each current state can transit to one of two possible next states depending weather the first stage of the memory register contains a 0 or a 1 . From Table 3 we see that. for example, if the current state is 100 , it means that the three rightmost stages of the memory register are occupied with 1,0 , and 0 respectively. Now, the outputs $n_{1}$ and $n_{2}$ of the encoder depend if the very first stage of the memory register is occupied by a 0 or 1 . If the first stage contains a 0 , then the output $\mathrm{n}_{1}=1$ and $\mathrm{n}_{2}=1$. Similarly, if the first stage contains a 1 , then the output $\mathrm{n}_{1}=\mathbf{0}$ and $\mathrm{n}_{2}=$ 0 . The next state depends on the occupancy of the first stage as well. In this case, if the first stage has a 0 in it, the next state is 010 (after shifting all bits to the right by one), and if the first stage contains a 1 , the next state is 101 .

\subsubsection{Trellis Diagram}

The trellis diagram is characterized with row states as well as with column states. The trellis diagram requires $2^{\mathrm{m}-1}$ nodes to represent $2^{\mathrm{m}-1}$ possible encoder states, where $\mathrm{m}$ is, once again, the number of stages of the memory register that determines the state of the encoder.

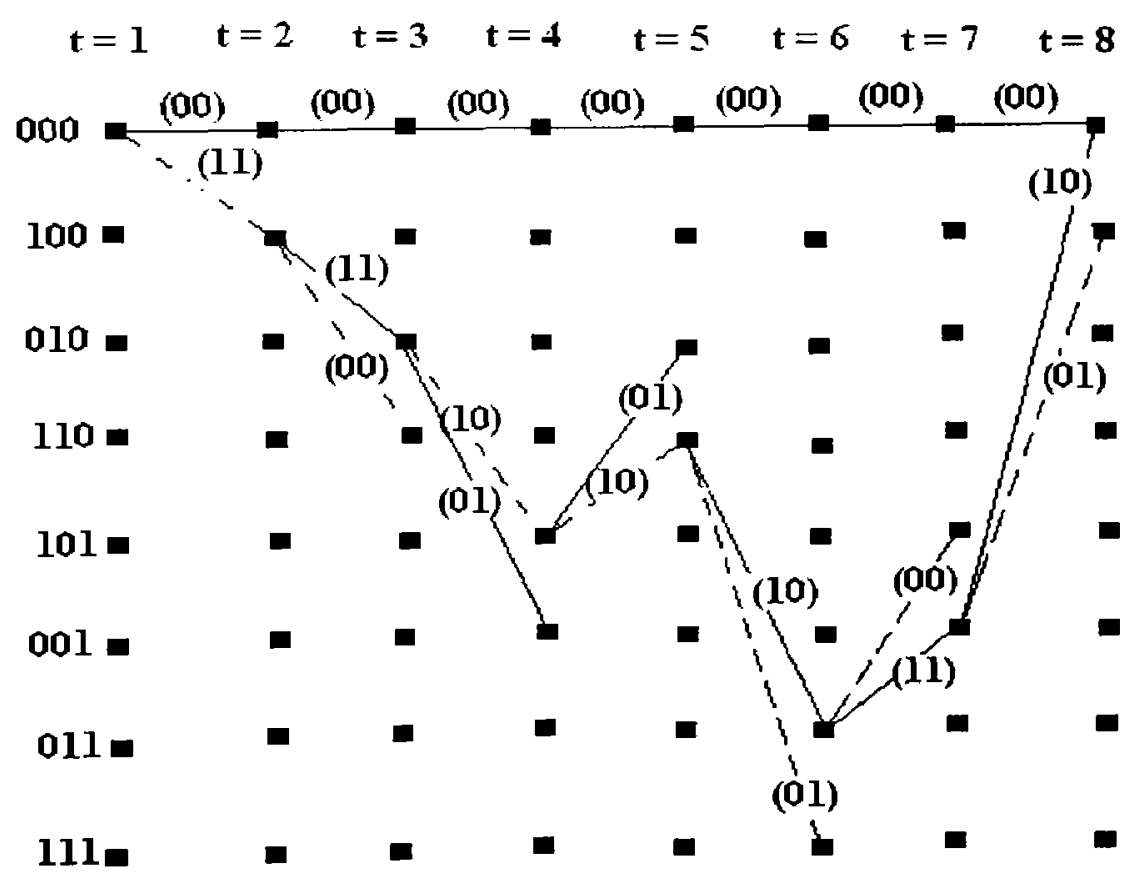

Figure 11: Trellis Diagram Representation of $(2,1,4)$ Convolutional Encoder. 
Figure 11 shows the resulting trellis diagram for the encoder in Figure 10 when the input sequence is 1011 . In the trellis diagram the transition from one state to another state upon the arrival of a 1 is represented by a dashed line. The transition cased by a 0 at the input of the encoder is pictured by a solid line. From Figure 11 we see that if the current state is 000 and a bit 1 comes in, the encoder reaches the state 100 . The output values for $n_{1}$ and $\mathrm{n}_{2}$ are 1 and 1 respectively.

\subsubsection{Convolutional Code Decoding}

If we assume that 3 bits are encoded using $(2,1,4)$ convolutional encoder in Figure 10 , then the output code sequence from the encoder will contain 6 bits, since each input bit is represented by 2 bits at the output of the encoder. These 6 bits may or may not have errors. We know from the encoding procedure that these bits are uniquely mapped. That means that a 3 bit sequence will have a unique 6 bit output. But due to errors, we can receive any of all possible combinations of 6 bits. The permutation of 3 input bits results in eight possible input sequences. Each of these input sequences has a unique mapping to a six bit code sequence by the encoder. These form a set of permissible sequences and the decoder's task is to determine which code word has been sent. Table 4 summarizes what has just been explained:

\begin{tabular}{|c|c|c|c|}
\hline Input & Valid Code Sequence & Received Sequence & Humuming Distance \\
\hline 000 & 000000 & 111100 & 4 \\
001 & 000011 & 111100 & 6 \\
010 & 001111 & 111100 & 4 \\
011 & 001100 & 111100 & 2 \\
100 & 111110 & 111100 & 1 \\
101 & 111101 & 111100 & 1 \\
110 & 110001 & 111100 & 3 \\
111 & 110010 & 111100 & 3 \\
\hline
\end{tabular}

Table 4: Humming Distance Used for Decoding Convolutional Codes.

Let's say we have received the following sequence: 111100 . As it can be seen from Table 4 the received sequence does not match to any of the 8 valid code sequences. How do we decode the received sequence? There are two approaches: we can do a correlation between each valid code sequence and the received sequence and pick the sequence with the best correlation. This approach is known as the soft decision decoding. The second approach deals with the term the Hamming Distance introduced earlier when we 
discussed decoding of linear block codes. As we have defined it, the Hamming distance between two code words is defined to be the number of elements in which those two code words differ. In our case we compute the Humming distance between the received word and all possible valid code words and choose the code word with the smallest Hamming distance. This is basically what is behind the so-called hard decision decoding. As the number of bits to be transmitted increases, the number of calculations required to be done during the process of decoding (Humming distance) significantly increases such that it is no longer practical to do decoding this way. This brings a need to find a more efficient method that does not examine all options and has a way of resolving ambiguity such as in cases when we have two or more code words have the same minimal Hamming distance (in Table 4, both 111110 and 111101 valid code sequences have the Hamming distance of 1 ) Then, which one to choose? To conclude; a method of decoding is needed in which for a message containing $\mathrm{m}$ bits we do not have to check each of $2^{\mathrm{m}}$ possible code words to determine which valid code word has been transmitted. This is where Viterbi decoding algorithm comes along.

\subsubsection{Viterbi Decoding Algorithm}

Viterbi decoding is the best known implementation of the maximum likelihood decoding. The maximum likelihood concept is a common sense way to make decisions when the statistical knowledge of the probabilities is available. In other words, the decoder chooses a valid code word $c$, as the transmitted sequence, if the likelihood $P(r \mid c)$ is greater than the likelihood of all the other valid code words. The Viterbi algorithm had been designed with the following two assumptions kept in mind:

1. the probability of occurrence of a single error is very small;

2. the probability of occurrence of two errors in a row is even much smaller than the probability of occurrence of a single error;

What these two assumptions mean is that in communication systems errors occur infrequently and that they are randomly distributed. The Viterbi decoder examines an entire received sequence of a given length and then the decoder computes a metric for each path and makes a decision based on this metric. The most common metric used is the Hamming distance metric. All paths are followed until two paths converge on one node. Then the path with the lower metric is kept and the one with higher metric is discarded. The paths selected are called the survivors. The early rejection of the unlikely paths greatly reduces the decoding complexity. Even though the Viterbi decoding algorithm solves the problems of unnecessary checking all of the $2^{m}$ possible code 
words, as well as the ambiguity when two code words have the same Hamming distance, it can not be consider as the ideal. It can be only considered as the best decoding algorithm among the existing ones. In some aspects of decoding it does overpower other decoding algorithms but it self has severe constrains imposed on by hardware complexity.

The Viterbi decoding algorithm uses the trellis diagram to perform the decoding procedure. However, the decoder's trellis diagram is a bit different than the encoder's trellis diagram. For the decoder's trellis it is much more convenient to label each trellis path at time $\mathbf{t}$ (path between any two states in the trellis) with the Hamming distance between sequence received at the time $t$ and the corresponding code word. It is important to realize that the encoder's trellis is known a priory to both the encoder and the decoder.

To fully understand the operation of the Viterbi algorithm, let's assume that the following stream of bits have been received at the receiver side and is to be decoded by the Viterbi algorithm:

received sequence: 11110111010111 .

Since the decoder has the knowledge about the encoder, it knows that the encoder is a $(2,1,4)$ convolutional encoder. That gives a clue to the decoder that the received sequence of bits has to be partitioned into subsequences where each subsequence will contain 2 bits (since each input bit at the transmitter side is encoded into a code word of 2 bits). So, the rearranged received sequence would now look like this: 11110111010111 . The decoder trellis diagram along with the respectful metric values is shown in Figure 12.

Looking at Figure 12, we see that all output pairs (bits $n_{1} n_{2}$ ) have been replaced by the metric values (Hamming distance values). For instance, the metric value for the path that represents the transition from the state 000 to 100 is equal 0 . That means that the output of the encoder and the received sequence at time $t=1$ are the same (Hamming distance is equal to zero if all bits on a received word and the corresponding code word are the same). Similarly, for the transition from 000 to 000 (when 0 arrives at the input of the encoder) the metric is equal to 2 . The meaning is that the received sequence and the code sequence at that particular state differ in 2 bits. Going back to the state diagram shown in Table 3 we can see that for the state 000 the code sequence (output bits), when a 0 arrives, is 00 . From the Figure 12, we see that the received sequence at time $t=1$ is 11 . Thus, words 00 and 11 differ in both their bits, thus the corresponding metric is therefore equal to 2 . 


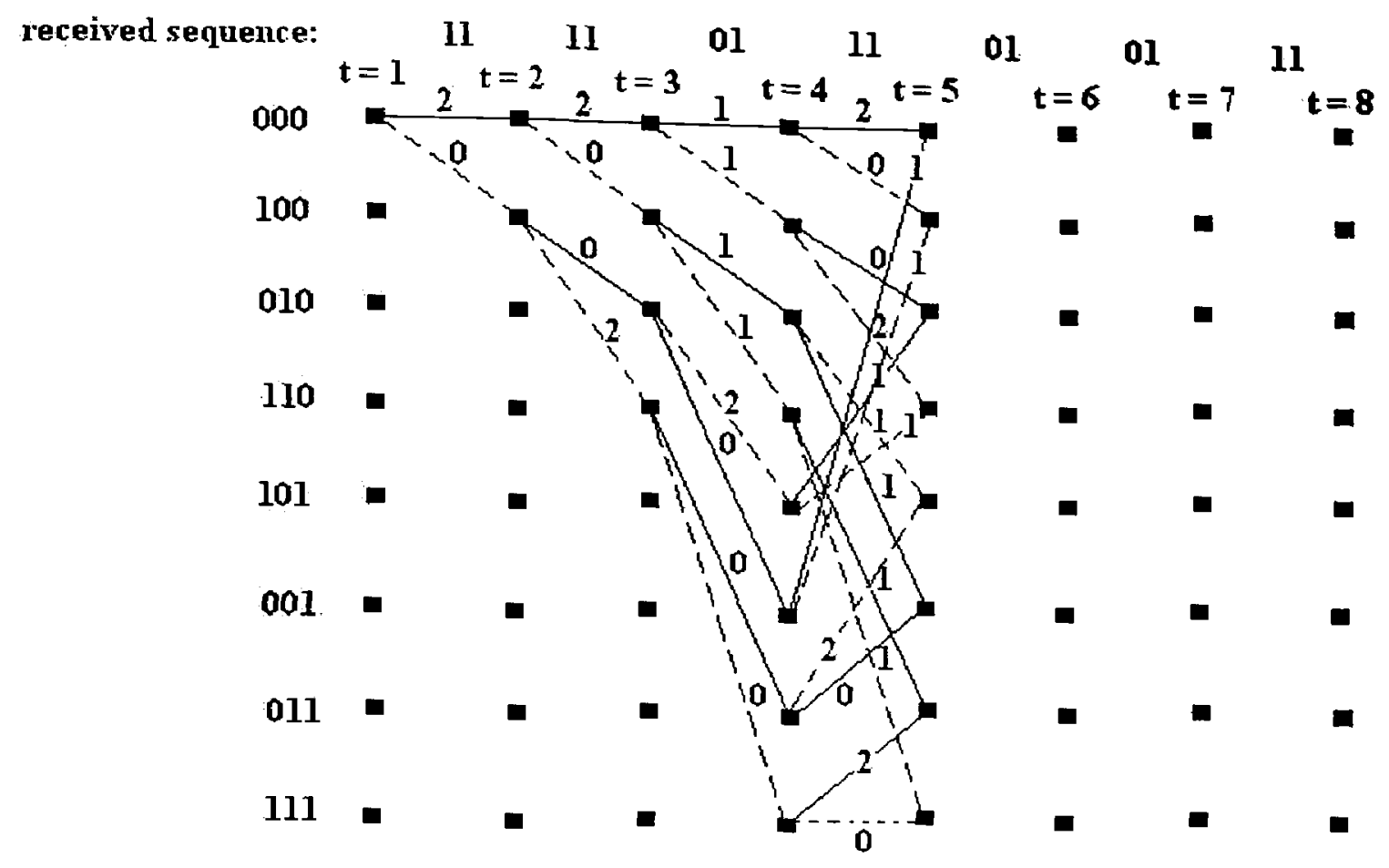

Figure 12: The Decoder Trellis Diagram (with Hamming Distances).

At time $t=5$ there are path crossing in every possible state (column nodes). These is where the Viterbi algorithm will get rid of some paths and reduce the complexity of the decoding which, as seen from Figure 12, already has become quite complex even though only 5 time units have elapsed.

Let's start with the state 000 (first node in the $t=5$ column). Two paths merge in this node. The first path is $000,000,000,000$, and $000 \mathrm{w}$ ith the metric equals to $7(2+2+1+2)$. The second path is $000,100,010,001$, and 000 having the metric value of $1(0+0+0+1)$. The algorithm will keep the second path since its cumulative metric is smaller than the cumulative metric of the first path. The second node (state 100) has the following two paths that get merged in the node. The first one is: $000,000,000,000,001$ with the metric equals to $5(2+2+1+0)$, and the second one: $000,100,010,001,100$ with the metric value of $1(0+0+0+1)$. The algorithm will select the second path and discard the first one due to the fact that the first path's metric is higher than the second path's.

Similarly, the algorithm will choose only 8 survivors and the trellis tree will have the same number of branches as it did at the $t-1$ unit of time. The resulting trellis diagram is shown in Figure 13. There we see that the path that has been running through the 000 states (first row) has completely disappeared and that for the time $t=1$ only one state 
transition remains; from 000 to 100 with metric 0 . If we now go back to the state diagram in Table 3, we can conclude that transition from 000 to 100 happens when a 1 is at the input of the encoder ( 1 is encoded). Therefore, the decoder decodes the first code word (sequence) to be a 1 .

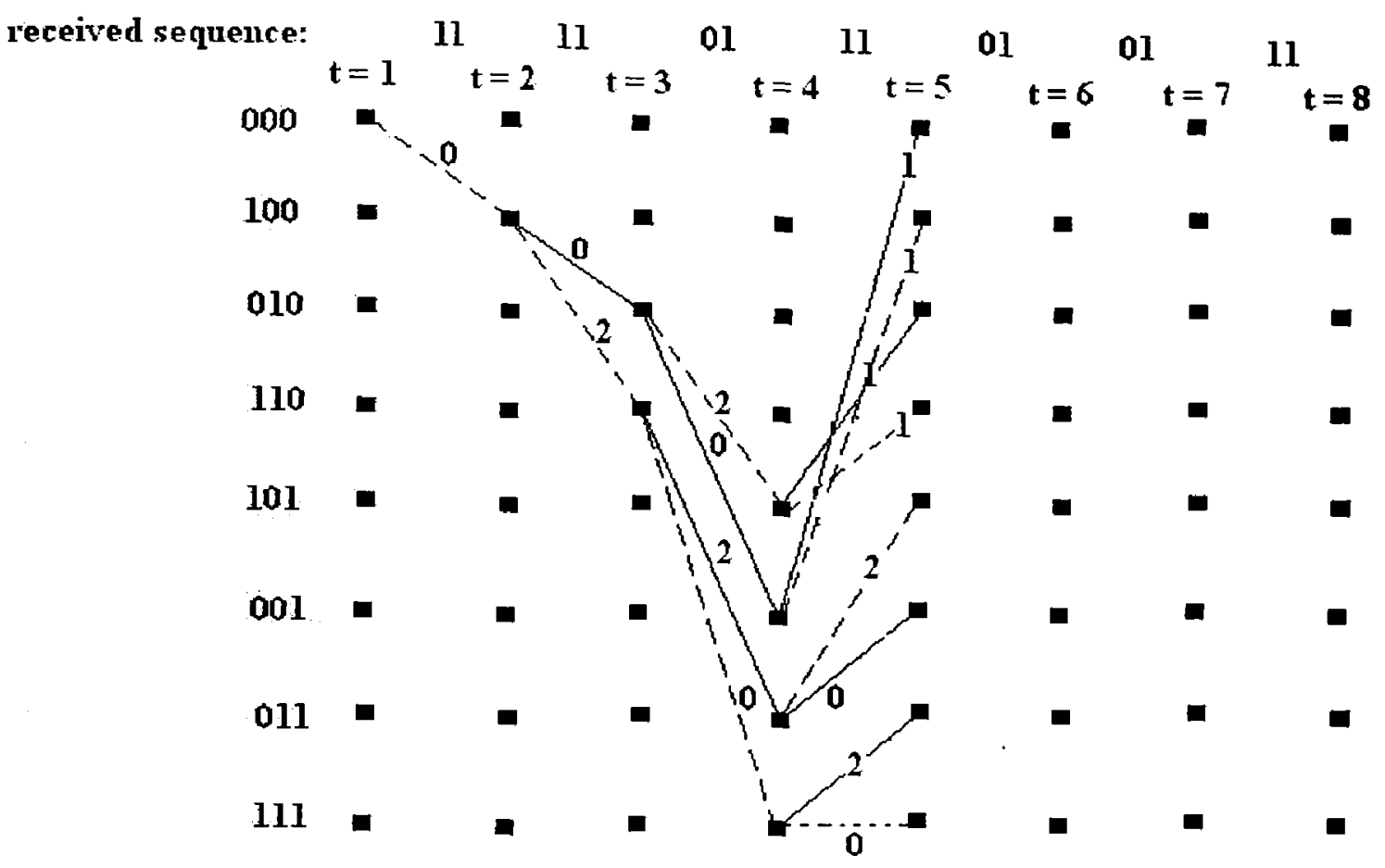

Figure 13: The Decoder Trellis Diagram after $t=5$ Time Units.

It is interesting to notice that it has taken 5 time units before the decoder could pull the decision that a 1 has been received at the time $t=1$. These 5 time units represent a decoding delay which can be as much as five times the constraint length (if $m=4$, the delay is 20 time units). This decoding process will continue until the last transmitted code word is decoded. In general, when a binary convolutional code with the constraint length $\mathrm{K}$ is decoded by Viterbi algorithm, there are $2^{\mathrm{K}-1}$ states as well as $2^{\mathrm{K}-1}$ surviving paths at each stage. Furthermore, a convolutional code with $\mathrm{k}$ bits being shifted in the encoder at a time will generate a trellis with $2^{\mathrm{k} \cdot(\mathrm{K}-1)}$ states. At each stage of the trellis, there are $2^{k}$ paths that get merged at each node. Only one path survives and this is the most probable (minimum distance) path. Thus, the number of computations in decoding performed at each stage increases exponentially with $\mathrm{k}$ and $\mathrm{K}$. This is a main reason why the use of Viterbi algorithm in decoding convolutional codes is troubled. Another disadvantage of the Viterbi decoding is that the decoding delay is usually too long for most practical applications, which often is not acceptable. Also, the memory required to 
store the surviving sequences would be of a humongous size and can be very expensive. The solution to this problem would be to modify the Viterbi algorithm in a way that results in a fixed decoding delay without significantly affecting the optimal performance of the algorithm. Viterbi decoding is quite important since it also applies to decoding of block codes. This form of trellis decoding is also used for Trellis-Coded Modulation (TCM).

\subsubsection{Matlab Implementation of the $(2,1,4)$ Convolutional Code}

Two Matlab files simulate the processes of convolutional encoding and decoding. The Matlab file "ConvolEncoder" performs the encoding procedure implementing a $(2,1,4)$ convolutional encoder. The file does all three types of encoding which have been discussed in sections above: Impulse Response, Polynomial, and Trellis. The file "Viterbi_Decoder.m" performs the decoding algorithm as being discusses in previous section. The decoding delay has been set to 20 time units.

At the end of this section, let's show the print out as the result of running an example code implementing the $(2,1,4)$ convolutional code in Matlab (the file "ConvolExample.m" is listed in Append ix B of the report):

$>$ ConvolExample

Data to be encoded:

1011

Connection Representation

Coded: 11111010101110

Polynomial Representation

Codeword: 11111010101110

Trellis Representation

Encoded Stream: 11111010101110

Decoded Sequence: 1011

Difference between Decoded and Encoded Sequences (Using Viterbi Decoding Algorithm): 0 


\section{Chapter 5: WCDMA}

\subsection{WCDMA Technology}

The first generation of mobile communication systems is represented by the analog systems designed to carry the voice application traffic. Those systems are mostly based on the analog frequency modulation technique and all systems employ the Frequency Division Multiple Access (FDMA) channelization technique to share the medium for the transmission of information. In FDMA, the transmission medium is divided among all stations which share the same allocated frequency bandwidth called the spectrum. The spectrum is divided into $N$ channels where $N$ represents the number of stations sharing the medium. Each of $\mathrm{N}$ stations transmits its information continuously on an assigned channel and during the period of the transmission no two stations share the same channel. Bandpass filters are used to contain the transmitted energies within assigned channels. For the reasons mentioned above, FDMA is suitable for stream traffics and finds its use in connection oriented systems. On the other hand, FDMA is extremely inefficient for bursty traffics since during the periods of no traffic the channel will sit idle, essentially a wasted resource.

The second generation of mobile communication systems relies exclusively on digital modulation techniques and two channelization techniques: Time Division Multiple Access (TDMA) and Code Division Multiple Access (CDMA). In TDMA, each station transmits during its assigned time slot and uses the entire spectrum during its transmission. In the basic form of TDMA, each station is assigned the same size time slot, $1 / \mathrm{N}$ for $\mathrm{N}$ stations, so each station could approximately have the same transmission bit rate. However, the stations' bit rate can be made variable by allocating several slots to the same channel or by changing the duration of time slots. Nevertheless, in TDMA the bit rate allocated to a station is static and this medium access approach, like FDMA, is not very desirable for bursty traffic.

Both FDMA and TDMA can be used to connect to a base station or a controller in two ways: using frequency division duplexing (FDD) and time division duplexing (TDD). FDD provides two distinct bands of frequencies for each station (two channels), the forward band (forward channels) that provides traffic from the base station to other stations, and the reverse band (reverse channels) that provides traffic from the stations to the base station. The frequency separation between each forward and reverse channel is constant throughout the system regard less of the particular channel being used (usually 
$45 \mathrm{MHz}$. TDD uses time instead of frequency to provide a forward and a reverse link. In TDD, each station has both a forward and a reverse time slot to facilitate bidirectional communication. If the time separation between the forward and reverse time slot is small, then the communication between the base station and other stations seems to be simultaneous. The FDMA channelization technique is used with FDD, while the TDMA channelization technique can be used with either FDD or TDD. CDMA provides another type of channelization technique. In TDMA and FDMA the transmission from different stations is clearly separated either in time or in frequency. The CDMA technique combines the TDMA and FDMA techniques and the transmissions from different stations occupy the entire frequency (like TDMA) at the same time (like FDMA).

\subsubsection{Direct Sequence Spread Spectrum}

Transmissions from different stations are separated with the use of the Direct Sequence Spread Spectrum (DSSS) method where a baseband data, before being transmitted, is modulated into a signal that occupies a much larger bandwidth than the original data. The technique is conceptually quite simple; the data is modulated via the XOR operation with a much higher data rate spreading code. Figure 14 shows an example of the DSSS method:
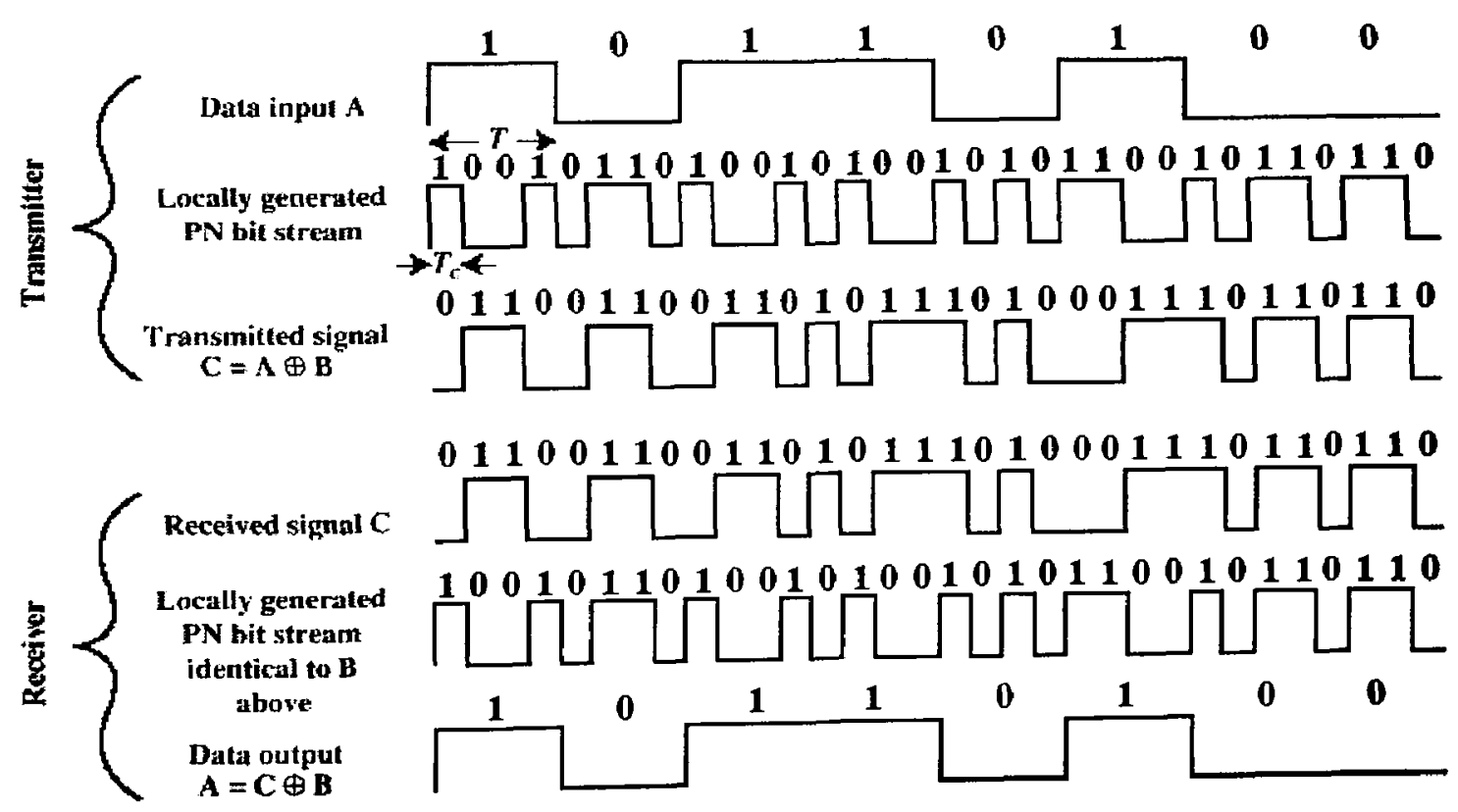

Figure 14: Direct Sequence Spread Spectrum. 
The spreading code is generated by a pseudo-random number generator. The spreading code only appears to be randomly generated but is in fact known to both the transiver and receiver. It can be very long, even infinitely long and must satisfy the following important properties; it has to be uniformly distributed, independent, and to have the correlation and cross-correlation property. The spread signal can not be detected by an eavesdropper unless they can generate the exact same spreading code and can synchronize its generation in time. The energy from the spread wideband signal within a narrow band is very low, and even if detected will appear to be a random noise. As more DSSS signals occupy a wideband channel the noise baseline will eventually increase until communications is no longer possible.

In general, the result of the DSSS process produces a very robust signal for transmission. We will go back to the CDMA technique once we introduce the third generation of mobile communications systems which use a new technology called Wideband CDMA (WCDMA).

The preceding discussion about DSSS requires that both the transmitter and receiver select very long random spreading codes ahead of time before they even begin to communicate. This is very impractical and another method that will provide an automated means of generating pseudo random spreading codes is needed. A simple generator polynomial (a shift register) used for the cyclic block codes can be modified to produce these spreading codes. Figure 15 shows a right shifted generator polynomial:

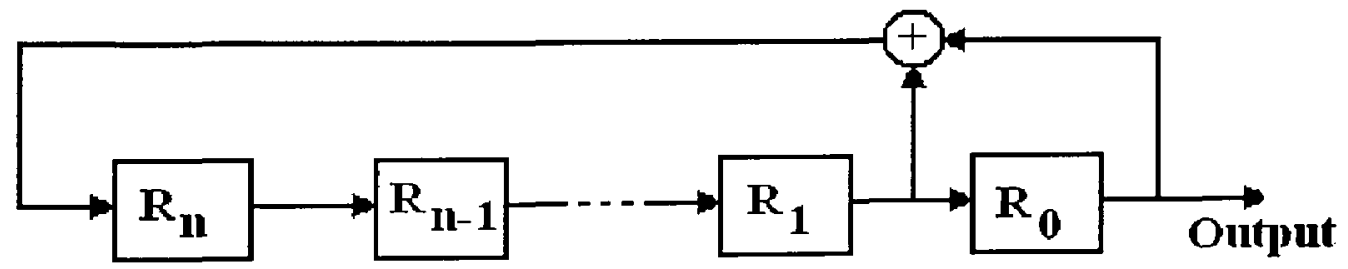

Figure 15: Right Shifted Generator Polynomial.

If the initial state of the registers $R_{n}, R_{n-1}, \ldots, R_{1}, R_{0}$ and appropriate feedback connections are selected, then the contents of the registers will cycle through all possible $2^{n}-1$ nonzero states before repeating. The resulting scquences are of the maximum length possible and can be considered to approximate random binary sequences in the sense that shifted version of a sequence are approximately uncorrelated. The length of the sequences depends on the number of the registers in the shifter and it is left to a designer to decide how many elements the sequences will have. Let's now take a look at the 
process of generating approximately random sequences by a right shifter that has 4 registers and whose initial state is: 1000 . The same shifter configuration shown in Figure 15 is used having 4 registers, from $R_{3}$ to $R_{0}$. By having the same hardware (right shifter) installed at both ends, the transmitter and receiver can generate the same sequences and synchronize them by implementing a constant delay. This is crucial to recover the received signals and extract them for the right channels:

\begin{tabular}{cccccc} 
Time & $\mathrm{R}_{3}$ & $\mathrm{R}_{2}$ & $\mathrm{R}_{1}$ & $\mathrm{R}_{0}$ & \\
\hline 0 & 1 & 0 & 0 & 0 & $\leftarrow$ Initial State \\
1 & 0 & 1 & 0 & 0 & \\
2 & 0 & 0 & 1 & 0 & \\
3 & 1 & 0 & 0 & 1 & \\
4 & 1 & 1 & 0 & 0 & \\
5 & 0 & 1 & 1 & 0 & \\
6 & 1 & 0 & 1 & 1 & \\
7 & 0 & 1 & 0 & 1 & \\
8 & 1 & 0 & 1 & 0 & \\
9 & 1 & 1 & 0 & 1 & \\
10 & 1 & 1 & 1 & 0 & \\
11 & 1 & 1 & 1 & 1 & \\
12 & 0 & 1 & 1 & 1 & \\
13 & 0 & 0 & 1 & 1 & \\
14 & 0 & 0 & 0 & 1 & \\
15 & 1 & 0 & 0 & 0
\end{tabular}

We can see that 15 different sequences are generated before the sequence 1000 repeats itself.

\subsubsection{Code Division Multiple Access}

The third generation of mobile systems is designed to support wideband services such as high speed Internet access, video and high quality image transmission along with the voice service. The WCDMA technology is developed in order to create a global standard for real time multimedia services. With the support of International Telecommunication Union (ITU) a specific spectrum was allocated $(2 \mathrm{GHz})$ used for sharing information among the third generation systems. The WCDMA systems use the CDMA medium access technique but a bit different than we have introduced in the 
earlier paragraphs. It goes a step further in the CDMA technology by using a so-called chip sequences instead of pseudo-random generated spreading code sequences.

Let's suppose that a data is generated at $R$ bits/seconds and that each bit in the data is transformed into $G$ bits by multiplying each bit (represented by either $a+1$ representing a 1 or a -1 representing a 0 ) by $G$ chip elements. All chip's elements are also represented by either $\mathbf{a}+1$ or $\mathbf{a}-1$. The chip sequence is produced by a special code and appears to be random except that it repeats after a very long period. The spreading factor $G$ is chosen in such a way that the transmitted signal occupies the entire spectrum. The chip sequences that are used for different channels must be selected so that any pair of chip sequences will have low cross-correlation. Otherwise, the receiver would not be able to separate the transmissions coming from stations using these chip sequences. One way to ensure that the cross-correlation between any pair of chip sequences will have low cross-correlation is to use the orthogonality property.

If we define two chip sequences, $A$ and $B$, both with the same number of elements $\mathbf{n}$ as:

$$
\begin{aligned}
& A=\left(a_{1}, a_{2}, a_{3}, \ldots, a_{n}\right), \quad \text { and } \\
& B=\left(b_{1}, b_{2}, b_{3}, \ldots, b_{n}\right)
\end{aligned}
$$

These two chip sequences are said to be orthogonal if their inner product, also called the dot product, is zero:

$$
A \cdot B=\sum_{i=1}^{n} a_{i} \cdot b_{i}=a_{1} \cdot b_{1}+a_{2} \cdot b_{2}+\cdots+a_{n} \cdot b_{n}=0
$$

Since each chip sequence consists from +1 and -1 elements, we can define that:

$$
\begin{aligned}
& A \cdot A=\sum_{i=1}^{n} a_{i}{ }^{2}=a_{1}{ }^{2}+a_{2}{ }^{2}+\cdots+a_{i}{ }^{2}=n, \\
& B \cdot B=\sum_{i=1}^{n} b_{i}{ }^{2}=b_{1}{ }^{2}+b_{2}{ }^{2}+\cdots+b_{j}{ }^{2}=n .
\end{aligned}
$$

If we now assume that we were sending a binary 0 in the channel that uses the chip sequence $A$, and a binary $I$ in the channel that uses the chip sequence $B$. The binary 0 spread out by the chip sequence $A$ will be: 


$$
0=-A=\left(-a_{1},-a_{2}, \ldots,-a_{n}\right)
$$

while the binary 1 spread out by the chip sequence $B$ will be:

$$
1=B=\left(b_{1}, b_{2}, \ldots, b_{n}\right) \text {. }
$$

The aggregate signal that will appear in the spectrum as the result of adding the spread versions of binary 0 and 1 is:

$$
T=\left(t_{1}, t_{2}, t_{3}, \ldots, t_{n}\right)=\left(-a_{1}+b_{1},-a_{2}+b_{2}, \ldots,-a_{n}+b_{n}\right)=-A+B
$$

When a receiver tries to recover the data sent through the channel with the chip sequence $A$, it will multiply the received signal $T$ with the chip sequence $A$ and then integrate (add) all the elements of the resulting sequence:

$$
\begin{aligned}
\sum_{i=1}^{n} t_{i} \cdot a_{i} & =t_{1} \cdot a_{1}+t_{2} \cdot a_{2}+\cdots+t_{n} \cdot a_{n}=T \cdot A=(-A+B) \cdot A \\
& =-A \cdot A+B \cdot A=-n+0=-n .
\end{aligned}
$$

Similarly, the data in the channel with the chip sequence B will be decoded as:

$$
\begin{aligned}
\sum_{i=1}^{n} t_{i} \cdot b_{i} & =t_{1} \cdot b_{1}+t_{2} \cdot b_{2}+\cdots+t_{n} \cdot b_{n}=T \cdot B=(-A+B) \cdot B \\
& =-A \cdot B+B \cdot B=0+n=n .
\end{aligned}
$$

Summarizing the results of the multiplying the received signal with the appropriate chip sequences, the receiver will determine that a binary 0 has been sent through the channel with the chip sequence $A(-n)$ and a binary 1 through the channel with the chip sequence $B$ (n).

Let's now take a look how we can choose chip sequences to use them within the same spectrum but for different channels. As we know from the discussion above, those chip sequences must be orthogonal among themselves. That means that the dot product of any chip sequence with itself will yield a positive number $n$, where $n$ is the number of elements in the chip sequence. Also, the dot product of any chip sequence with its complement would yields a negative number $n$. And finally the dot product of any sequence with any other sequence or their complements will yield a zero number. 


\subsubsection{Walsh Orthogonality}

One of the methods to obtain orthogonal chip sequences of length $n$, where $\mathrm{n}=2^{\mathrm{m}}$, is to use the Walsh matrix. The Walsh matrix is build from binary coefficients and is defined recursively as:

$$
\begin{gathered}
\mathrm{W}_{1}=[+1] \\
\mathrm{W}_{2 \mathrm{n}}=\left[\begin{array}{ll}
\mathrm{W}_{\mathrm{n}} & \mathrm{W}_{\mathrm{n}} \\
\mathrm{W}_{\mathrm{n}} & \bar{W}_{\mathrm{n}}
\end{array}\right],
\end{gathered}
$$

where $\bar{W}_{\mathrm{n}}$ is the complement of the element $\mathrm{W}_{\mathrm{n}}$., In some literature the Walsh matrix $W_{1}$ is defined as having its only element equal to -1 . Regardless of the type of the $W_{1}$ Walsh matrix being used, higher order Welsh matrices $(n=2,4,8, \ldots)$ provide a set of orthogonal sequences. It is straight forward to show the construction of the Walsh matrix for $n=2,4,8$ or any higher order Walsh matrix. The $W_{2}$ is formed by having matrices $\mathrm{W}_{1}$ and $\overline{\mathrm{W}}_{1}$ as its elements:

$$
W_{2}=\left[\begin{array}{ll}
+1 & +1 \\
+1 & -1
\end{array}\right] \text {. }
$$

Then, the $\mathrm{W}_{4}$ matrix is formed from $\mathrm{W}_{2}$ according to (42b):

$$
\mathrm{W}_{4}=\left[\begin{array}{llll}
+1 & +1 & +1 & +1 \\
+1 & -1 & +1 & -1 \\
+1 & +1 & -1 & -1 \\
+1 & -1 & -1 & +1
\end{array}\right]
$$

And finally, the $W_{8}$ matrix is formed from the $W_{4}$ matrix and its complement $\bar{W}_{4}$ : 


$$
\mathrm{W}_{8}=\left[\begin{array}{llllllll}
+1 & +1 & +1 & +1 & +1 & +1 & +1 & +1 \\
+1 & -1 & +1 & -1 & +1 & -1 & +1 & -1 \\
+1 & +1 & -1 & -1 & +1 & +1 & -1 & -1 \\
+1 & -1 & -1 & +1 & +1 & -1 & -1 & +1 \\
+1 & +1 & +1 & +1 & -1 & -1 & -1 & -1 \\
+1 & -1 & +1 & -1 & -1 & +1 & -1 & +1 \\
+1 & +1 & -1 & -1 & -1 & -1 & +1 & +1 \\
+1 & -1 & -1 & +1 & -1 & +1 & +1 & -1
\end{array}\right]
$$

Generally, some of the $n$ orthogonal sequences in the $W_{n}$ do not alternate quickly between +1 and -1 and as the result these sequences will not produce a transmitted signal that is spread over the whole available spectrum. Due to this fact, the Walsh matrices are primarily used to provide channelization while in practice the matrices are combined with other additional spreading such as DSSS.

All the time in our discussion about CDMA technology, we assumed that at the receiver end each received signal has the same power level. This is very important assumption and in order to work properly the CDMA technique requires all signals at a given receiver to have approximately the same power. If this is not the case, then transmissions from nearby stations could overwhelm transmissions from distant stations. This problem is called the near-far problem. The goal is that the receiver receives the same power level from all stations regardless of distance from the receiver (within a cell). If the power level from one station is higher than needed, the quality will be excessive thus taking a disproportionate share of the resources and generating unnecessary interference with signals from other stations. On the other hand, if the power level from a station is too low, it will result in poor quality of the received signal. In order to keep the received power at a suitable level, the WCDMA standard defines a fast power control that updates power levels from each station 1500 times every second.

Let's now take an example that will summarize everything that has been said regarding the CDMA technology:

\section{EXAMPLE (CDMA Channelization):}

A two channel system uses the following two orthogonal chip sequences (taken as the $3^{\text {rd }}$ and $7^{\text {th }}$ row from the Welsh matrix $\mathrm{W}_{8}$ :

$$
C_{-} A=(+1,+1,-1,-1,+1,+1,-1,-1) \text {, }
$$




$$
C_{-} B=(+1,+1,-1,-1,-1,-1,+1,+1) \text {. }
$$

Transmit bits: 100 on the channel $A$ and bits: 010 on the channel B. Recover the signal on the channel $A$.

\section{SOLUTION:}

To transmit a 0 through the channel $A$, the system will apply the complement of the chip sequence $C_{-} A$ and to transmit $a 1$, it will transmit the chip sequence $C_{-} A$ itself.

Similarly, to transmit a 0 through the channel $B$, the system will send out the complement of the chip sequence $C_{-} B$ and to transmit $a 1$, it will send out the chip sequence $C$ B.

For the channel A, bits 100 are spread before being transmitted as shown below:

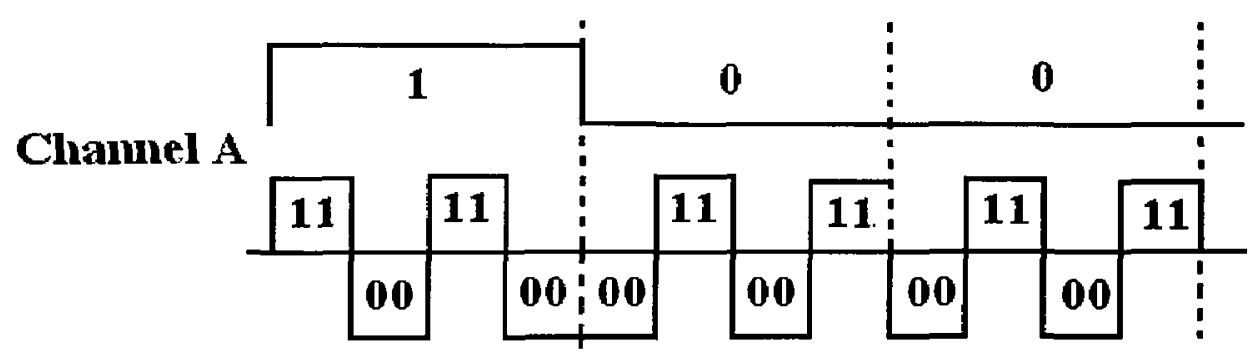

On the channel B, bits 010 are spread over the spectrum in the following manner:

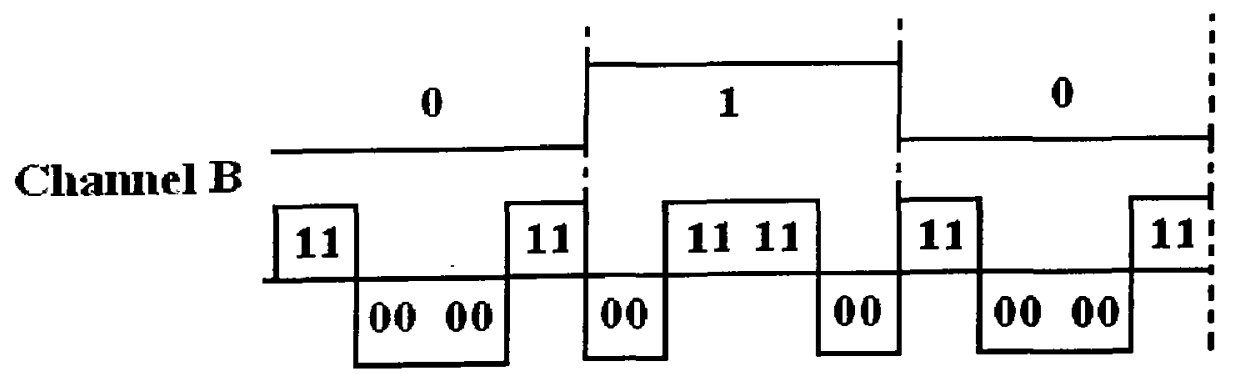

So, the data being spread at the channel $A$ is:

$$
A=(+1,+1,-1,-1,+1,+1,-1,-1,-1,-1,+1,+1,-1,-1,+1,+1,-1,-1,+1,+1,-1,-1,+1,+1) \text {, }
$$

while the spread data at channel B is: 


$$
B=(+1,+1,-1,-1,-1,-1,+1,+1,-1,-1,+1,+1,+1,+1,-1,-1,+1,+1,-1,-1,-1,-1,+1,+1) .
$$

The aggregate signal $(A+B)$ made up by adding the signals on the channels $A$ and $B$ look like this:

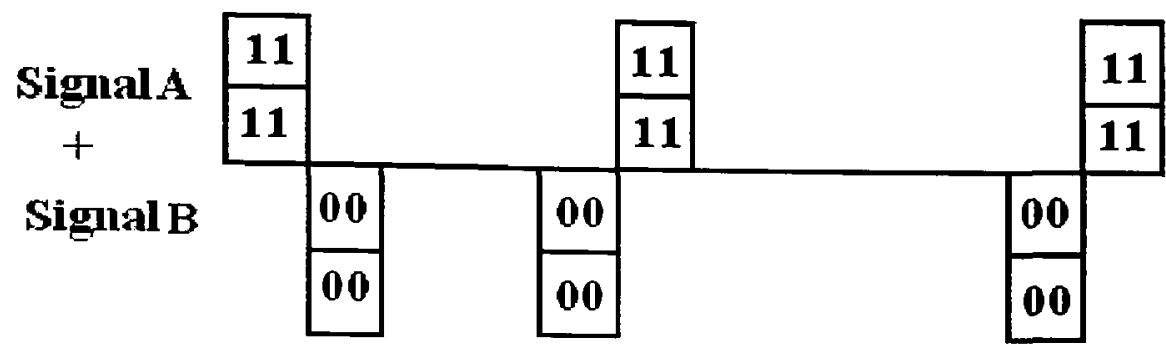

$$
A+B=(+2,+2,-2,-2,0,0,0,0,-2,-2,+2,+2,0,0,0,0,0,0,0,0,-2,-2,+2,+2) .
$$

At the receiver side, to recover the signal meant for the channel $A$ the aggregate signal is multiplied with the chip sequence $A$ at the correlator:

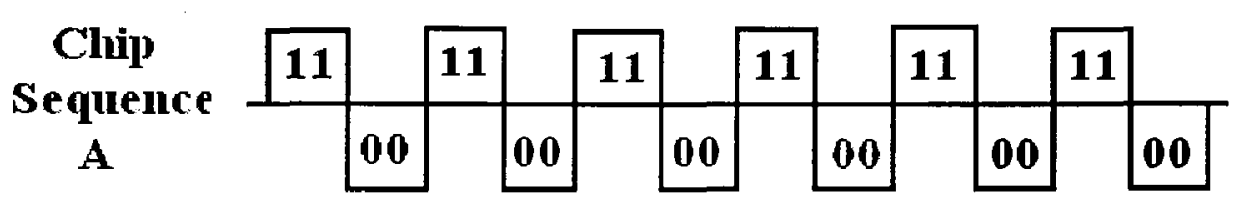

$$
\text { C_A }=(+1,+1,-1,-1,+1,+1,-1,-1,+1,+1,-1,-1,+1,+1,-1,-1,+1,+1,-1,-1,+1,+1,-1,-1) \text {; }
$$

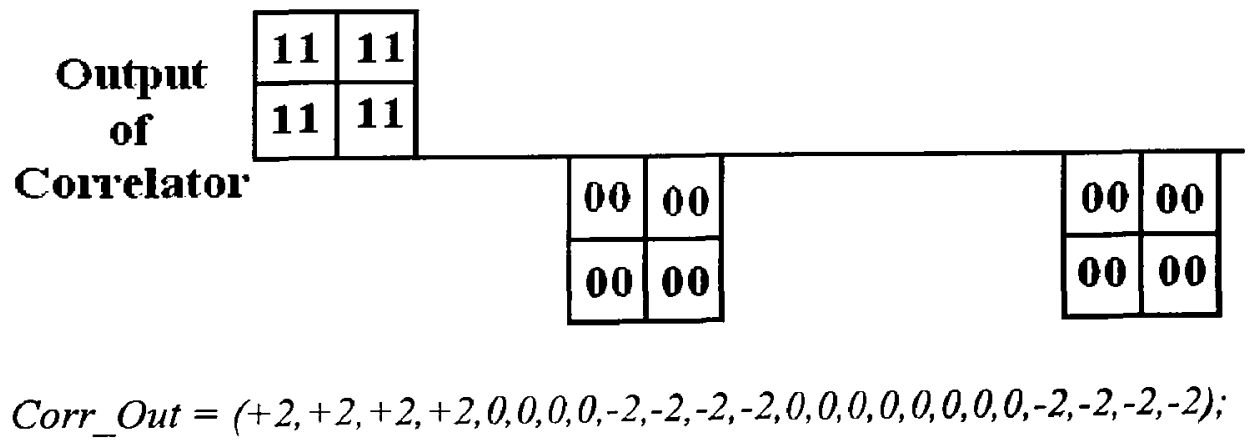

Now, at the integrator each sequence of 8 bits coming out of the correlator will be added due to the fact that each bit is represented (spread) by 8 bils (chip sequence). 


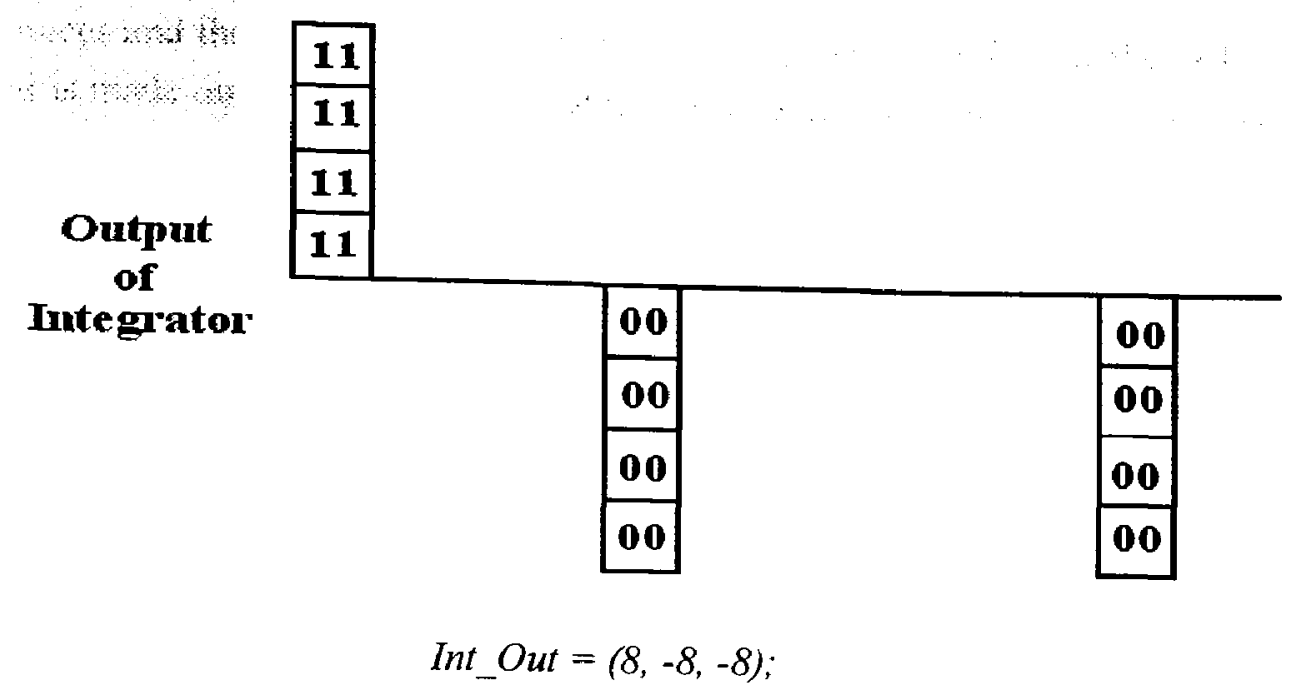

It is now easy to determine that the recovered signal on channel $A$ is:

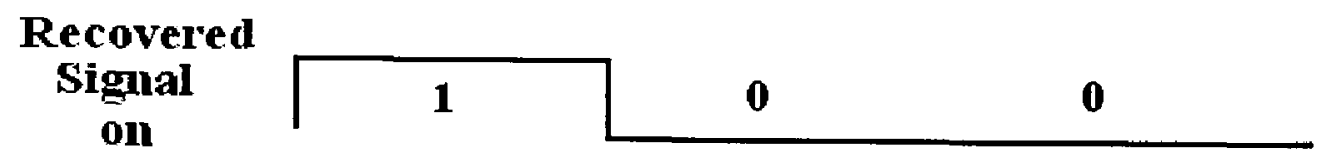

Chanmel A

\subsection{Matlab Implementation of WCDMA}

Six Matlab files implement the process of WCDMA. The main file "Aggreg_one.m" that contains calls for the routines called at various times during the execution of the program. It first calls the routine that create the Welsh matrix, $W_{8}$. Next, it performs the DSSS modulation. Then, the chip sequences for the channels are extracted from the Walsh matrix (rows 3 and 7). An aggregate signal is created next by performing the $\mathrm{A}+\mathrm{B}$ matrix operation. The noise is created and added to the aggregate signal that is at this point ready to be transmitted through the air. At the receiver end the received signal is multiplied with both the chip sequence $\mathrm{A}$ and chip sequence $\mathrm{B}$. The all elements of the resulting sequences are added up and a received bit is recovered (extracted). The Matlab function "Welsh_Matrix.m" that creates a Welsh matrix with the size $8 \times 8$. The Matlab function "Complement. $m$ " that creates the complements of the chip sequences A and B. The Matlab function "DSSS.m" that performs the Direct Sequence Spread Spectrum on both channels. The Matlab function "CDMA_Encode.m" in which each bit of data is represented (spread) by the chip sequence. A 1 is replaced by the chip sequence while a 0 is replaced by the complement of the chip sequence. And the last one, "CDMA_Decode.m" Matlab function in which the received signal is multiplied with the 
chip sequences and the resulting sequences are added up. The decision whether a 1 or a 0 is received is made on the following way: if the value obtained from the integrator is greater or equal to 4 , a bit 1 is recovered. Similarly, if the value is less or equal to -4 , a bit 0 is recovered. The values obtained between -4 and 4 give us the meaning that that part of the received signal is not meant for that particular channel;

At the end of the discussion about WCDMA let's take a look how the Matlab code works with the same input conditions as in the example above; bits on the channel A: 100 , bits and the channel B: 010 . Note that the Matlab code implements the DSSS modulation while the example above did not.

>> aggreg_one

Output from Correlator (channel A): $00-10-2-2-2-2$

Output from Integrated output: -9

Output from Correlator (channel A): 22220000

Output from Integrated output: 8

Output from Correlator (channel A): $-2-I-2-20000$

Output from Integrated output: -7

Output from Correlator (channel B): 0 0-102222

Output from Integrated output: 7

Output from Correlator (channel B): 22220000

Output from Integrated output: 8

Output from Correlator (channel B): :-2 -1 -2 -2 0000

Output from Integrated output: -7

Sent on channel A: 100

Recovered on channel A: 100

Sent on channel B: 010

Recovered on channel B: 010

Compare data sent \& recovered on channel A: 0

Compare data sent \& recovered on channel $B: 0$

We see that at the receiver the data for both channels $A$ and $B$ is recovered properly. One might notice that in the example above the values out of the integrator are 
always either 8 or -8 , while from the Matlab printout we can see that the values out of the integrator are not always either 8 or $\mathbf{- 8}$. This is due to the fact that the Matlab simulation mixes the aggregate signal with random no ise before the corrupted signal is decoded at the receiver end. In the example above we assumed that the transmission medium was noiseless. The same Matlab file "aggreg_one.m" used for the main simulation was used for the example. 


\section{Chapter 6: RESULTS}

In order to simulate the operation of the source-channel codec, two signals are transmitted through the system in Figure 1. On the channel A, a 4.99 second long speech file, 'speech.wav', saying: "The Discrete Fourier Transform of a real valued signal is conjugate symmetric", is applied. On the channel B, the image file, "world.jpg", representing a gray level still image with the resolution $102 \times 132$ is applied. Both signals are shown bellow:

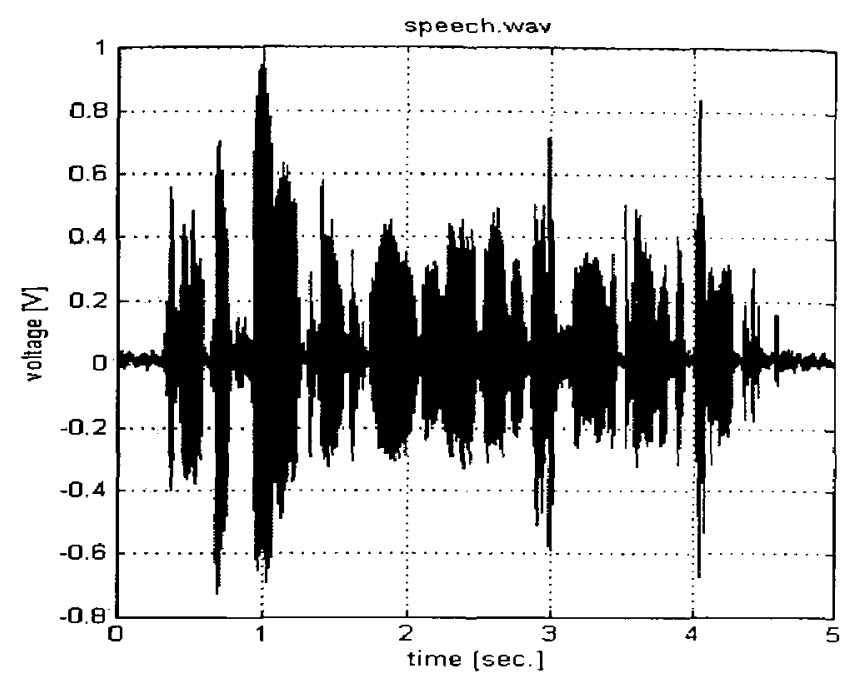

Figure 16: Speech Data.

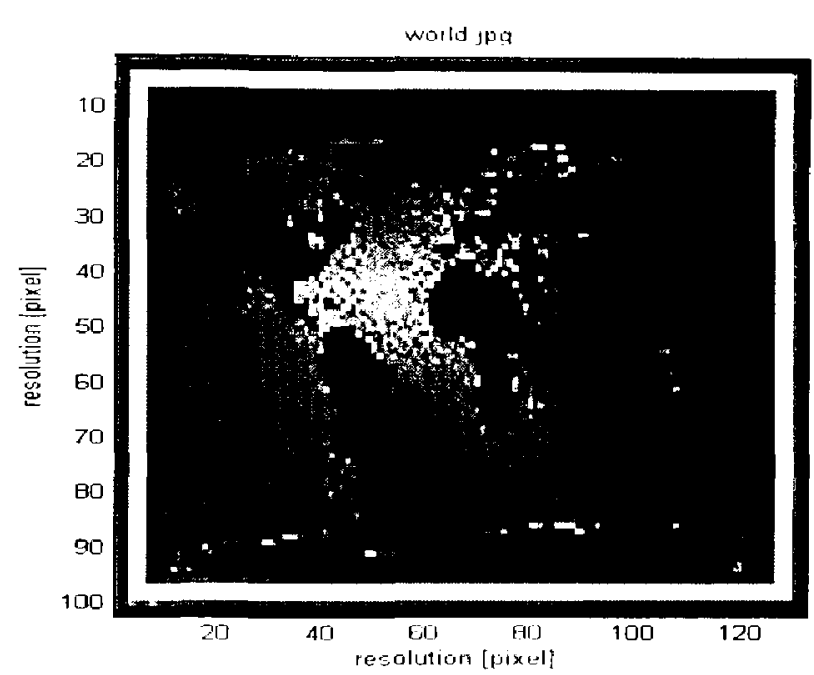

Figure 17: Still Image Data.

At the Formatting stage, speech signal on the Channel $\mathrm{A}$ is first sampled at the sampling frequency of $22050 \mathrm{~Hz}$ with the help of 'wavread' Matlab command. The sampled version of the speech signal contains 110,033 sample points. In order to explain how these numbers are obtained, we first plot the frequency spectrum of the speech signal as shown in Figure 18. From Figure 18 we see that the maximum frequency of the signal is $11,025 \mathrm{~Hz}$. Applying the Nyquist Sampling Rate criteria which states that the sampling frequency of an analog signal must be equal or greater than twice the maximum frequency of the signal, it is clear why the sample rate of the speech signal is $22,050 \mathrm{~Hz}$. Since the number of sampled points in one second is equal to the sampling frequency, we conclude that the sampled version of the speech signal would have 22,050 sampled points for one second. When that number is multiplied with 4.99 , which is the total duration of the speech signal, we get 110,033 sampled points for the ent ire speech signal. In further discussion the sampled version of the speech signal will simple be referred to as sampled signal. 


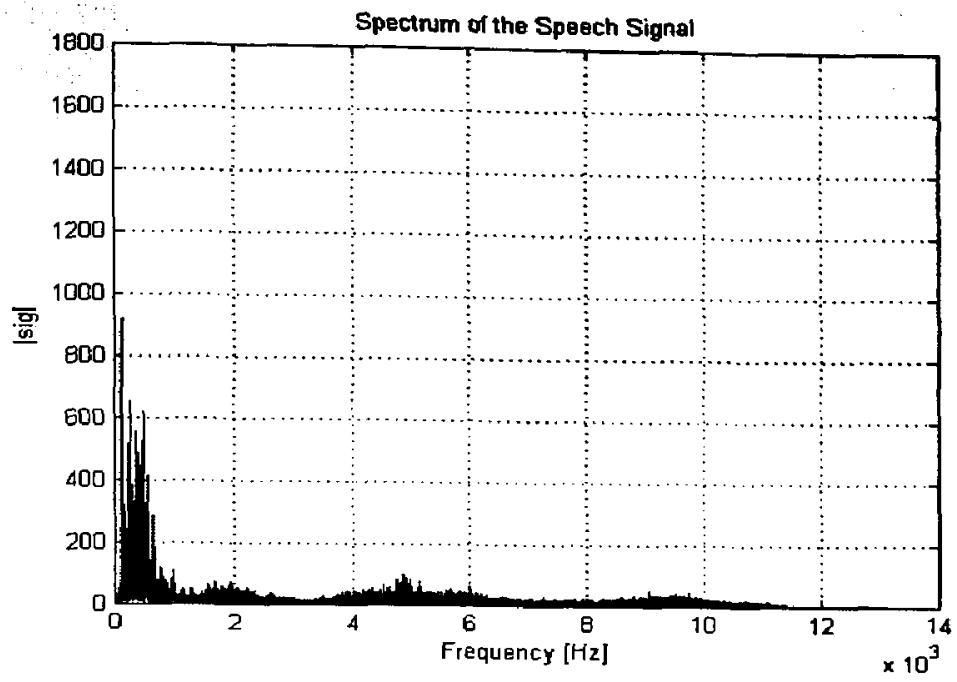

Figure 18: Frequency Spectrum of Speech Signal.

Next, the sampled signal is quantized to 256 levels since a speech signal requires 8 bits to represent each sample. The maximum value of the sampled signal is 0.9921 , while its minimum value is -0.7268 . Consequently, the quantile interval $q$ is 0.0067 . Since the 8 bit quantizer used in this project is a linear quantizer, every level in the quantizer (e.g. 256 levels) will be of the same size, the size of the quantile interval q. At this point we obtain the quantized signal as a result of the quantization.
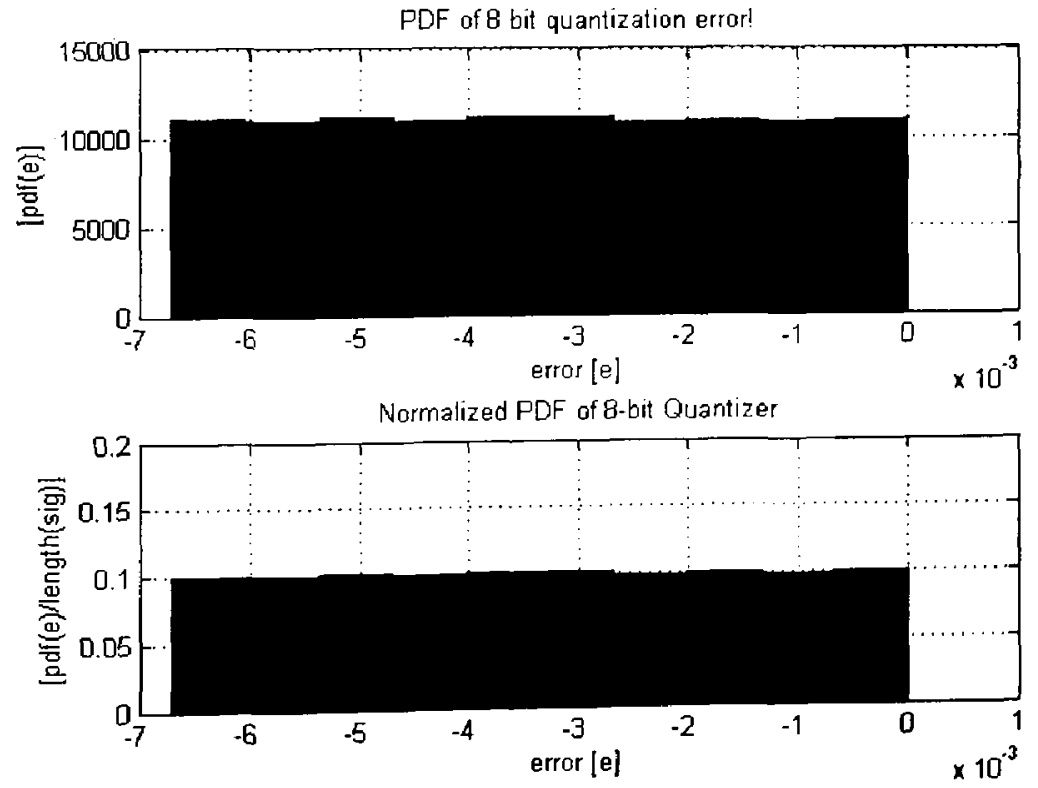

Figure 19: PDF of Quantization Error. 
To observe the quality of the quantization which is measured through the quantization signal to noise ratio (SNR)q, we first have to obtain the error caused by the quantization. The error is calculated as the difference between the sampled signal and the quantized signal. The probability density functions (PDF) of the quantization error as well as its normalized version are shown in Figure 19. The quantization signal to noise ratio is the ratio between the signal power and the noise power where, in this case, the noise power is the quantization error. The quantization error power can be found in two ways. The first one is to integrate the product of the squared difference of the quantization error and the its mean, with the probability density function of the quantization error (e.g. see the equation (11) in Chapter 2). The second way, which is used in this project, is to calculate the quantization error power as the mean of the squared error vector:

$$
\text { error_power }=\frac{\sum_{i=1}^{n} e_{i}{ }^{2}}{n} \text {, }
$$

where $\mathrm{e}$ is the quantization error vector and $\mathrm{n}$ is the size of the quantization error vector. To get the value in $[\mathrm{dB}] \mathrm{s}$ :

$$
\text { error_power }[\mathrm{dB}]=10 \cdot \log _{10} \text { (error_power). }
$$

The signal power is obtained exactly the same way but instead of the quantization error vector, the signal vector, containing the values of the samples signal, is used. Using Matlab, the quantization signal to noise ratio (SNR)q is then obtained to be:

$$
\mathrm{SNR}=30.9077[\mathrm{~dB}]
$$

If we recall from Chapter 2; a quick approximation of the (SNR)q for a quantizer could be made by multiplying the number of bits that each sample is represented with by $6 \mathrm{~dB}$. Applying that, the theoretical value of the (SNR)q in this project would be $48 \mathrm{~dB}$. The reason why we obtain much lower the (SNR)q is due to the fact that we have used a linear quantizer. In general, linear quantizers are wasteful for speech signals since many of the quant ization levels are rarely used. To support this claim a histogram given in Figure 20 shows the frequency of usage for each of 256 levels for our 8-bit uniform quantizer. It can be seen that the majority of the quantization levels used in the process falls in the following range $[96,125]$ of quantization levels. We also see that the quantization level 111 contains 9,164 sampled points which makes almost 10 percents of 
all sampled points in the sampled signal. A non-uniform quantizer would provide a better quantization and the quantization error would be made proportional to the signal size. The overall (SNR)q would improve by reducing the error for the predominant parts of the signal, at the expense of an increase in error for the rarely occurring parts of the signal.

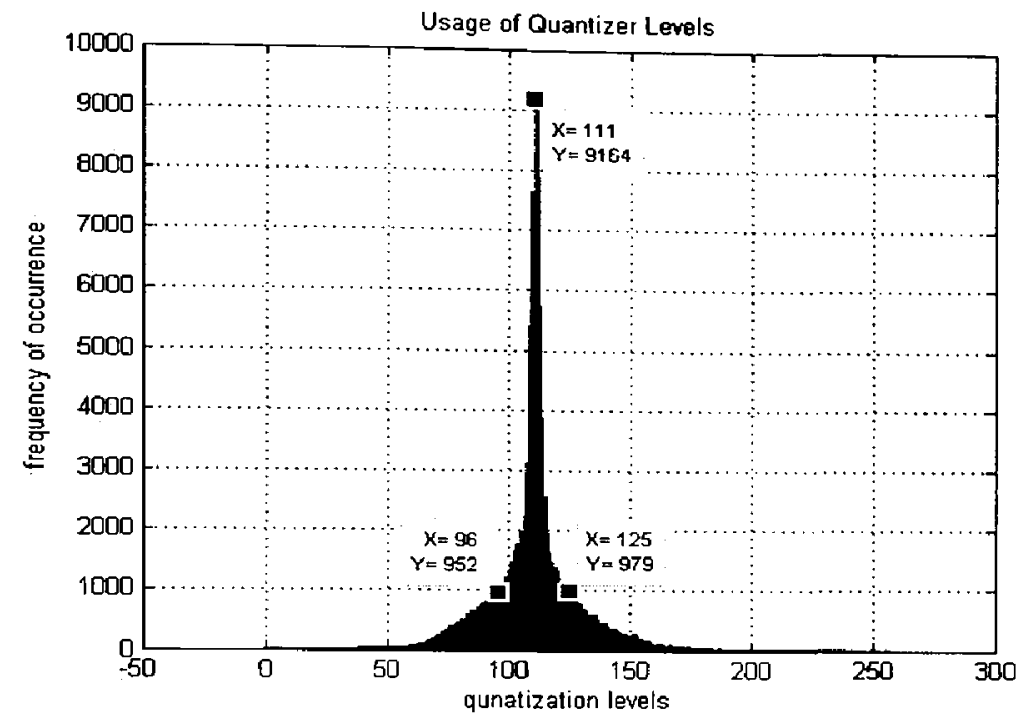

Figure 20: Frequency of Usage of Quantizer Levels.

The last part of formatting the speech signal is the Pulse Code Modulation, where the quantization level for each element of the sampled signal vector is converted to its binary representation. Here a simple decimal to binary conversion is used where the quantization level (decimal number) for each signal element is converted to 8 bit binary number. The obtained binary values are appended into a binary sequence that represents the digitized speech data. The data on the Channel A consists of 880,264 binary digits $(8 \times 110,033)$.

On the Channel B, the formatting stage for the image is much simpler compared to that of the speech signal. We only have to calculate the PDF of the image. The image is read into the Matlab's environment in the ASCII format. The PDF of the image, shown in Figure 21 , will give us the frequency of occurrence for each of 256 numbers that the intensities of the image's pixels are represented with. In the process of calculating the probability of occurrence for each number in the $[0,255]$ range, any number in the $[0,255]$ range that does not appear in the image matrix is considered as irrelevant since it carries no information relevant to our image. Once the frequency of occurrence of each number in the image matrix is calculated, it is divided by the total number of elements in the image matrix, forming the probability of occurrence for each number in the $[0,255]$ range that 
appears in the matrix. The $x$-axis lists numbers in the $[0,255]$ range showing all 256 possible intensities of the image. The $y$-ordinate lists the probabilities of occurrence for the intensity of each pixel that is present in the image.

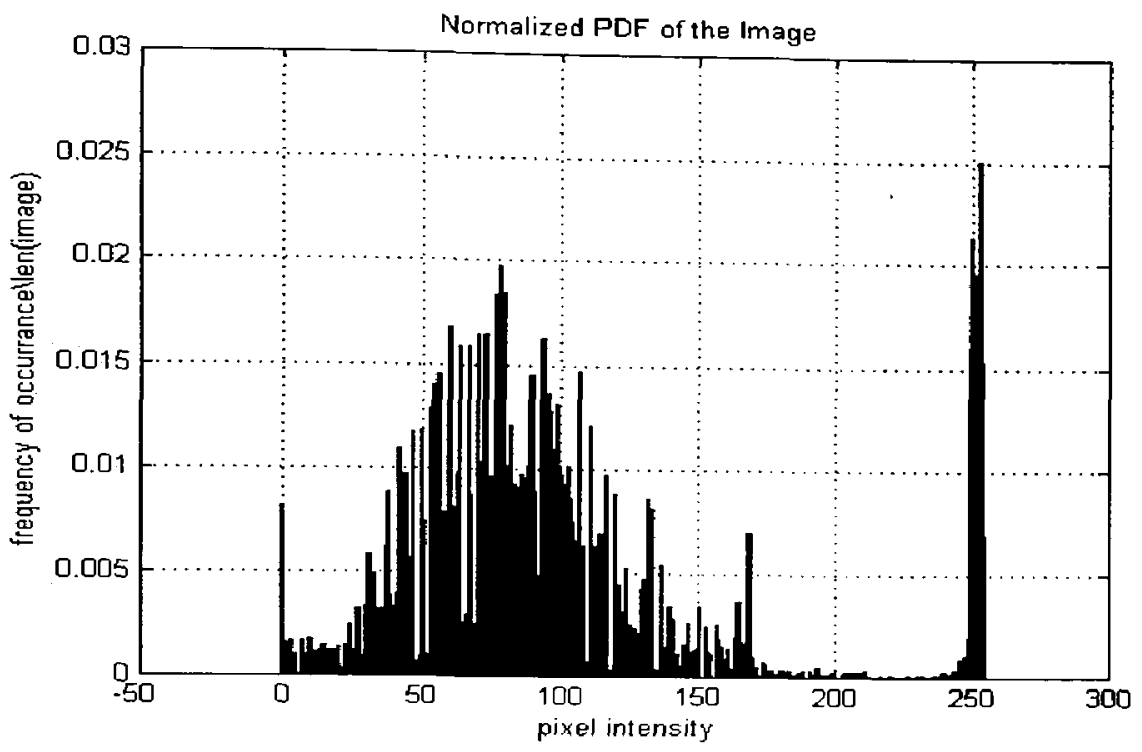

Figure 21: PDF of the Image.

The next stage in the system for both Channels is the source encoder stage. Channel $\mathbf{A}$ uses the Lempel-Ziv-Welch algorithm to perform the source encoding; to compress the digitized speech signal as much as possible. The principles of the Lempel-Ziv-Welch algorithm are explained in the Chapter 3 and here only the Lempel-Ziv-Welch table is presented as the result of the Matlab simulation. Since the table has 45705 entries, it would be impossible to list all of them here in this report. However, the first 50 entries will be listed, thus a reader can get a feeling how the algorithm has performed the operation. During the implementation of this algorithm, I've taken a liberty to change the way that the code word column is implemented. Instead of having a fixed-length implementation of the code word ( 12 bits per code word), I've used a variable-length implementation. In the real world this change would require a bit more complex decoder. The software implementation of the decoder only requires knowledge of how many bits are used to encode every single code word. This is solved by adding another column in the Lempel-Ziv-Welch table called code word length that provides the information to the decoder about how many bits are used to encode each code word. Let's take a look at the first 50 entries of the Lempel-Ziv-Welch table obtained through the Matlab simulation: 


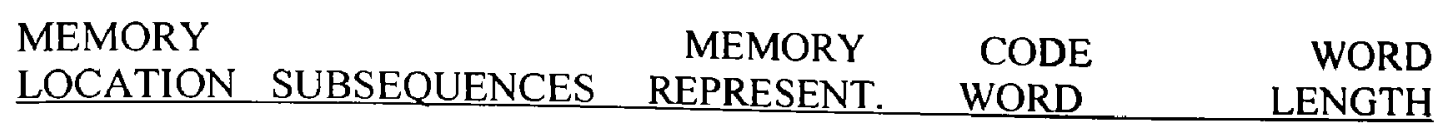

\begin{tabular}{|c|c|c|c|c|}
\hline 1 & 0 & 0 & - & - \\
\hline 2 & 1 & 1 & - & - \\
\hline 3 & 01 & 1 & 11 & 2 \\
\hline 4 & 10 & 2 & 100 & 3 \\
\hline 5 & 11 & 2 & 101 & 3 \\
\hline 6 & 010 & 3 & 110 & 3 \\
\hline 7 & 110 & 5 & 1010 & 4 \\
\hline 8 & 1101 & 7 & 1111 & 4 \\
\hline 9 & 011 & 3 & 111 & 3 \\
\hline 10 & 0110 & 9 & 10010 & 5 \\
\hline 11 & 101 & 4 & 1001 & 4 \\
\hline 12 & 1011 & 11 & 10111 & 5 \\
\hline 13 & 0101 & 6 & 1101 & 4 \\
\hline 14 & 10110 & 12 & 11000 & 5 \\
\hline 15 & 101101 & 14 & 11101 & 5 \\
\hline 16 & 1010 & 11 & 10110 & 5 \\
\hline 17 & 11011 & 8 & 10001 & 5 \\
\hline 18 & 01011 & 13 & 11011 & 5 \\
\hline 19 & 01101 & 10 & 10101 & 5 \\
\hline 20 & 011011 & 19 & 100111 & 6 \\
\hline 21 & 010110 & 18 & 100100 & 6 \\
\hline 22 & 11010 & 8 & 10000 & 5 \\
\hline 23 & 110110 & 17 & 100010 & 6 \\
\hline 24 & 1011011 & 15 & 11111 & 5 \\
\hline 25 & 0101101 & 21 & 101011 & 6 \\
\hline 26 & 10101 & 16 & 100001 & 6 \\
\hline 27 & 1011010 & 15 & 11110 & 5 \\
\hline 28 & 1101101 & 23 & 101111 & 6 \\
\hline 29 & 0110110 & 20 & 101000 & 6 \\
\hline 30 & 10110111 & 24 & 110001 & 6 \\
\hline 31 & 00 & 1 & 10 & 2 \\
\hline 32 & 110111 & 17 & 100011 & 6 \\
\hline 33 & 101101111 & 30 & 111101 & 6 \\
\hline 34 & 0110111 & 20 & 101001 & 6 \\
\hline 35 & 1011011110 & 33 & 1000010 & 7 \\
\hline 36 & 1101111 & 32 & 1000001 & 7 \\
\hline 37 & 0111 & 9 & 10011 & 5 \\
\hline 38 & 000 & 31 & 111110 & 6 \\
\hline 39 & 001 & 31 & 111111 & 6 \\
\hline 40 & 10111 & 12 & 11001 & 5 \\
\hline 41 & 10110111101 & 35 & 1000111 & 7 \\
\hline 42 & 101110 & 40 & 1010000 & 7 \\
\hline 43 & 01101110 & 34 & 1000100 & 7 \\
\hline
\end{tabular}




$\begin{array}{lllll}44 & 011011100 & 43 & 1010110 & 7 \\ 45 & 1101110 & 32 & 1000000 & 7 \\ 46 & 0110111001 & 44 & 1011001 & 7 \\ 47 & 1011100 & 42 & 1010100 & 7 \\ 48 & 11011100 & 45 & 1011010 & 7 \\ 49 & 110111001 & 48 & 1100001 & 7 \\ 50 & 101111 & 40 & 1010001 & 7\end{array}$

Once the table is completed, the code words from the fourth column are appended to each other creating a binary sequence; a new compressed binary representation of the speech data. The new binary sequence contains 684,407 binary digits, which makes the compression ratio of $22 \%$ for the speech signal. What is interesting for the Lempel-ZivWelch algorithm is that when the input sequence is longer, the compression ratio is better (higher).

On the channel B, the image data is source encoded with Huffman Coding Algorithm. As it has been mentioned in Chapter 3, Huffman Coding Algorithm provides a systematic procedure for constructing uniquely decodable variable-length code words that are efficient in the sense that the average number of bits per source is minimized. Since we have 256 possible intensities for each pixel, it means that we have 256 (from 0 to 255) possible symbols in the source alphabet. Here is the result of the Matlab simulation which assigns a variable-length code word to each of these 256 symbols as per discussion in Chapter 3. The alphabet symbols and their respective binary representations are listed in the left-to-right up-to-down manner.

$\begin{array}{llll}\mathrm{S} 0=1001111011 & \mathrm{~S} 3=10001110001 & \mathrm{~S} 7=10001110010 & \mathrm{~S} 13=1001011000 \\ \mathrm{~S} 14=1001011001 & \mathrm{~S} 15=1001011010 & \mathrm{~S} 17=10001110011 & \mathrm{~S} 18=1001011011 \\ \mathrm{~S} 19=10001110100 & \mathrm{~S} 20=100111110 & \mathrm{~S} 21=101100011 & \mathrm{~S} 22=10001110101 \\ \mathrm{~S} 23=10001110110 & \mathrm{~S} 24=1011001 & \mathrm{~S} 25=100111111 & \mathrm{~S} 26=100000010 \\ \mathrm{~S} 27=10100101 & \mathrm{~S} 28=110110010 & \mathrm{~S} 29=10001010 & \mathrm{~S} 30=10001110111 \\ \mathrm{~S} 31=11110001 & \mathrm{~S} 32=110110011 & \mathrm{~S} 33=10001011 & \mathrm{~S} 34=10111000 \\ \mathrm{~S} 35=1001011100 & \mathrm{~S} 36=10001111000 & \mathrm{~S} 37=10100110 & \mathrm{~S} 38=100000011 \\ \mathrm{~S} 39=10100111 & \mathrm{~S} 40=1010110 & \mathrm{~S} 41=100000100 & \mathrm{~S} 42=10001100 \\ \mathrm{~S} 43=10001101 & \mathrm{~S} 44=100000101 & \mathrm{~S} 45=10000101 & \mathrm{~S} 46=1111111 \\ \mathrm{~S} 47=10111001 & \mathrm{~S} 48=10101000 & \mathrm{~S} 49=10111010 & \mathrm{~S} 50=1101101 \\ \mathrm{~S} 51=11110010 & \mathrm{~S} 52=11110011 & \mathrm{~S} 53=10000000 & \mathrm{~S} 54=1011111 \\ \mathrm{~S} 55=10111011 & \mathrm{~S} 56=10111100 & \mathrm{~S} 57=1101110 & \mathrm{~S} 58=10101001 \\ \mathrm{~S} 59=100000110 & \mathrm{~S} 60=10000110 & \mathrm{~S} 61=10000111 & \mathrm{~S} 62=11110100 \\ \mathrm{~S} 63=10001000 & \mathrm{~S} 64=1111101 & \mathrm{~S} 65=10111101 & \mathrm{~S} 66=111001000\end{array}$




\begin{tabular}{|c|c|c|c|}
\hline $67=10001001$ & $S 68=111001001$ & $S 69=111001010$ & $S 70=101000000$ \\
\hline $71=10101010$ & $S 72=1001011101$ & $S 73=11110101$ & $S 74=111001011$ \\
\hline $75=111001100$ & $S 76=1001011110$ & $S 77=11011000$ & $S 79=1001011111$ \\
\hline $80=101000001$ & $S 81=10001111001$ & $S 82=1001100000$ & $S 83=111001101$ \\
\hline $84=11110110$ & $S 85=111001110$ & $S 86=111001111$ & $S 87=101000010$ \\
\hline $88=10001111010$ & $S 89=10001111011$ & $S 90=111010000$ & $S 92=10001111100$ \\
\hline$S 94=10001111101$ & $S 95=10001111110$ & $S 96=100000111$ & $S 97=1001100001$ \\
\hline $98=111010$ & $\mathrm{~S} 100=1$ & $S 10 l=10001111111$ & $S 103=111010010$ \\
\hline $105=111010011$ & $S 107=10010000000$ & $S 108=10010000$ & $S 109=1001100011$ \\
\hline$S 110=1001100100$ & $S 111=1001100101$ & $S 112=11$ & S113= \\
\hline $114=$ & $\mathrm{S} 115=1 \mathrm{C}$ & & $S 117=1$ \\
\hline $19=10$ & $\mathrm{~S} 120=11$ & $S 121=111010110$ & $\mathrm{~S} 122=1$ \\
\hline $123=10010000011$ & $\mathrm{~S} 124=101000100$ & $S 126=1001$ & $\mathrm{~S} 130=11$ \\
\hline $131=1001101 \mathrm{C}$ & $\mathrm{S} 132=1001$ & $\mathrm{~S} 133=$ & $\mathrm{S} 134=$ \\
\hline S135 & $\mathrm{S} 136$ & & $\mathrm{~S} 138=1$ \\
\hline $\mathrm{S} 140$ & $\mathrm{~S} 141=1$ & $\mathrm{~S} 14$ & $S 143=1$ \\
\hline 00 & $\mathrm{~S} 145=1$ & $147=10011010$ & $\mathrm{~S} 148=10$ \\
\hline S149 & $\mathrm{S} 15$ & 1101 & S154= \\
\hline$S 155=10$ & S $157=101$ & $\mathrm{~S} 158=10$ & $\mathrm{~S} 159=1$ \\
\hline $\mathrm{S} 160=11$ & $S 161=10010001$ & $S 164=10$ & $S 165=1001$ \\
\hline $166=10$ & $S 169=10010010011$ & $S 170=111011100$ & $S 171=10011$ \\
\hline$S 173=10010$ & $S 174=1001110010$ & 01 & 110 \\
\hline$S 177=10$ & $S 180=10010011000$ & $S 181=10010011$ & $\mathrm{~S} 182=$ \\
\hline $\mathrm{S} 183=1001110011$ & $S 185=1001110100$ & S1 86 $=10010011011$ & $\mathrm{~S}: 87=10010011100$ \\
\hline$S 190=10010011101$ & $S 191=1001110101$ & $S 194=101000111$ & $S 196=10010011110$ \\
\hline$S 197=10010011111$ & $S 198=10010100000$ & $S 199=10010100$ & $S 201=101001000$ \\
\hline $202=111011101$ & $S 204=111011110$ & $S 205=10010100010$ & $S 208=101001001$ \\
\hline $209=111011111$ & $S 210=10010100011$ & $S 211=100001001$ & $S 213=10010100100$ \\
\hline $\mathrm{S} 215=10010100101$ & $S 217=11111001$ & $S 218=10010100110$ & $S 219=1001110110$ \\
\hline$S 222=10010100111$ & $S 223=101100010$ & $S 224=10010101000$ & $S 226=10010101001$ \\
\hline$S 227=1001110111$ & $\mathrm{~S} 228=111100000$ & $\mathrm{~S} 233=1001111000$ & $\mathrm{~S} 234=1001111001$ \\
\hline$S 236=10010101010$ & $S 237=10010101011$ & $S 238=10010101100$ & $\mathrm{~S} 239=10010101101$ \\
\hline$S 240=10010101110$ & $S 241=111100001$ & $S 242=10010101111$ & $\mathrm{~S} 243=10001110000$ \\
\hline $244=1010111$ & $S 245=10101011$ & $S 246=1101111$ & $S 247=1111110$ \\
\hline $\mathrm{S} 248=111000$ & $S 249=10110000$ & $S 251=11010$ & $\mathrm{~S} 252=101101$ \\
\hline $253=1001111010$ & $S 254=1100$ & $S 255=0$ & \\
\hline
\end{tabular}


Average Code Length: 3.482

Entropy of the Source $\mathrm{H}(\mathrm{x})=3.42933$

Efficiency $=98.4872 \%$

At this point in the system, the image data is represented by 8,705 binary digits. The efficiency as the ratio between the entropy and the average code length is $98.48 \%$, which is considered to be an almost maximum compression.

The following stage in the source-channel codec system is the channel encoding. As discussed in Chapter 4, the purpose of the channel encoder is to introduce, in a control manner, some redundancy in the data binary sequence that can be used at the receiver to overcome the effects of noise and interference encountered in the transmission of the signal through the channel. In other words, redundancy in the data binary sequence aids the receiver in decoding the desired data sequence. The Channel A uses the Linear Block Code algorithm to add redundant bits to the data binary sequence obtained from the source encoder stage. The $(12,8)$ type of Linear Block Code is used, meaning that every 8 bits from the data binary sequence will be transformed into a 12 bit sequence, thus adding 4 redundant bits. To show the ability of the code to detect and correct errors, as per the discussion in Chapter 4, a Bit Error Rate (BER) versus Signal to Noise Ratio (SNR) plot is obtained and shown in Figure 22.

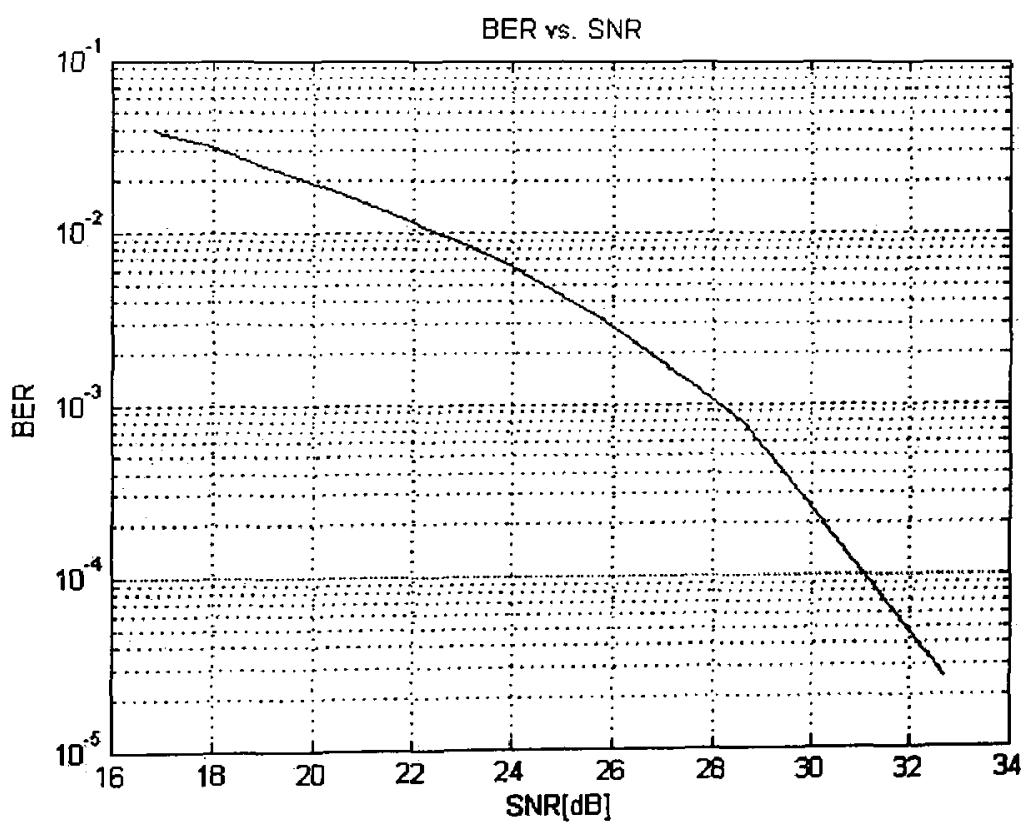

Figure 22: BER vs. SNR for $(12,8)$ Linear Block Code. 
The Bit Error Rate is defined as the number of errors not being corrected by the decoder divided by the total number of bits in the input sequence to the Linear Block Code system. The Signal to Noise Ratio is, as before, defined as the ratio between signal power and the noise power. Four points are plotted on the graph obtained for four different values of the noise. From the plot we see that the first uncorrected errors occur while the SNR is still relatively high $(\sim 33 \mathrm{~dB})$. This would lead us that the code is not sufficiently capable of performing error corrections since the BER vs. SNR curve from the reference $[2, \mathrm{pp} 267]$ starts having first undetected errors around $\mathrm{I} 0 \mathrm{~dB}$.

The reason for the early appearance of uncorrected errors lies in the fact that the Linear Block Code $(12 \times 8)$ used in the simulation can detect and correct only $2^{n-k}-1=15$ error patterns (see Chapter 4 ). That means that only 12 single bit error patterns plus three 2 bit error patterns can be detected (in the project a single bit error patterns are detectable and correctable only). To improve the performance of the code, more error patterns must be detected and corrected which requires the longer time execution of the code (it takes 5 hours for Matlab to generate $(12 \times 8)$ code's BER vs. SNR). However, in the real world of communications errors happen approximately 1 in $10^{6}$ transmitted bits and the possibility of having 2 errors in a row are very rare. Here are the main matrices obtain in the Matlab simulation for the $(12,8)$ Linear Block Code:

$\mathbf{P}=\left[\begin{array}{llll}1 & 0 & 1 & 0 \\ 1 & 1 & 0 & 0 \\ 1 & 0 & 1 & 1 \\ 1 & 1 & 0 & 1 \\ 1 & 1 & 1 & 0 \\ 0 & 1 & 0 & 1 \\ 0 & 1 & 1 & 1 \\ 0 & 1 & 1 & 0\end{array}\right] ;$
$\mathbf{H t}=\left[\begin{array}{llll}1 & 0 & 0 & 0 \\ 0 & 1 & 0 & 0 \\ 0 & 0 & 1 & 0 \\ 0 & 0 & 0 & 1 \\ 1 & 0 & 1 & 0 \\ 1 & 1 & 0 & 0 \\ 1 & 0 & 1 & 1 \\ 1 & 1 & 0 & 1 \\ 1 & 1 & 1 & 0 \\ 0 & 1 & 0 & 1 \\ 0 & 1 & 1 & 1 \\ 0 & 1 & 1 & 0\end{array}\right] ;$

$G=\left[\begin{array}{llllllllllll}1 & 0 & 1 & 0 & 1 & 0 & 0 & 0 & 0 & 0 & 0 & 0 \\ 1 & 1 & 0 & 0 & 0 & 1 & 0 & 0 & 0 & 0 & 0 & 0 \\ 1 & 0 & 1 & 1 & 0 & 0 & 1 & 0 & 0 & 0 & 0 & 0 \\ 1 & 1 & 0 & 1 & 0 & 0 & 0 & 1 & 0 & 0 & 0 & 0 \\ 1 & 1 & 1 & 0 & 0 & 0 & 0 & 0 & 1 & 0 & 0 & 0 \\ 1 & 1 & 1 & 0 & 0 & 0 & 0 & 0 & 1 & 0 & 0 & 0 \\ 1 & 1 & 1 & 0 & 0 & 0 & 0 & 0 & 1 & 0 & 0 & 0 \\ 0 & 1 & 0 & 1 & 0 & 0 & 0 & 0 & 0 & 1 & 0 & 0 \\ 0 & 1 & 1 & 1 & 0 & 0 & 0 & 0 & 0 & 0 & 1 & 0 \\ 0 & 1 & 1 & 0 & 0 & 0 & 0 & 0 & 0 & 0 & 0 & 1\end{array}\right] ; ;$




\begin{tabular}{|c|c|c|c|c|c|c|c|c|c|c|c|c|c|c|c|}
\hline$e=[0$ & 0 & 0 & 0 & 0 & 0 & 0 & 0 & 0 & 0 & 0 & 0 & $\mathrm{Se}=[0$ & 0 & 0 & 0 \\
\hline 1 & 0 & 0 & 0 & 0 & 0 & 0 & 0 & 0 & 0 & 0 & 0 & 1 & 0 & 0 & 0 \\
\hline 0 & 1 & 0 & 0 & 0 & 0 & 0 & 0 & 0 & 0 & 0 & 0 & 0 & 1 & 0 & 0 \\
\hline 0 & 0 & 1 & 0 & 0 & 0 & 0 & 0 & 0 & 0 & 0 & 0 & 0 & 0 & 1 & 0 \\
\hline 0 & 0 & 0 & 1 & 0 & 0 & 0 & 0 & 0 & 0 & 0 & 0 & 0 & 0 & 0 & 1 \\
\hline 0 & 0 & 0 & 0 & 1 & 0 & 0 & 0 & 0 & 0 & 0 & 0 & 1 & 0 & 1 & 0 \\
\hline 0 & 0 & 0 & 0 & 0 & 1 & 0 & 0 & 0 & 0 & 0 & 0 & 1 & 1 & 0 & 0 \\
\hline 0 & 0 & 0 & 0 & 0 & 0 & 1 & 0 & 0 & 0 & 0 & 0 & 1 & 0 & 1 & 1 \\
\hline 0 & 0 & 0 & 0 & 0 & 0 & 0 & 1 & 0 & 0 & 0 & 0 & 1 & 1 & 0 & 1 \\
\hline 0 & 0 & 0 & 0 & 0 & 0 & 0 & 0 & 1 & 0 & 0 & 0 & 1 & 1 & 1 & 0 \\
\hline 0 & 0 & 0 & 0 & 0 & 0 & 0 & 0 & 0 & 1 & 0 & 0 & 0 & 1 & 0 & 1 \\
\hline 0 & 0 & 0 & 0 & 0 & 0 & 0 & 0 & 0 & 0 & 1 & 0 & 0 & 1 & 1 & 1 \\
\hline 0 & 0 & 0 & 0 & 0 & 0 & 0 & 0 & 0 & 0 & 0 & 1 ]; & 0 & 1 & 1 & 0 ]; \\
\hline
\end{tabular}

The Channel B applies a different algorithm for error detection of the $(2,1,4)$ Convolutional Code. It is called the Viterbi algorithm. For this algorithm the measure of efficiency of the code cannot be established through the BER vs. SNR since the Viterbi algorithm guaranties that any input sequence will be correctly decoded as long as the decoding delay is sufficiently long. The decoding delay used in this project is 20 . The principles of the Convolutional Code algorithm as well as those of the Viterbi algorithm are fully presented in Chapter 4 .

The last stage on the transmitter side is the WCDMA stage that consists from Direct Sequence Spread Spectrum modulation and CDMA channelization (Medium Access Control). First, each signal is XOR-ed with different pseudo-random generated sequences and that each bit of these new obtained signals is represented by the chip sequences. Two chip sequences obtained from the Welsh matrix are used. For Channel A:

$$
\mathrm{C}_{-} \mathrm{A}=\left[\begin{array}{llllllll}
+1 & +1 & -1 & -1 & +1 & +1 & -1 & -1
\end{array}\right] \text {; }
$$

and for the Channel B:

$$
\text { C_B }=\left[\begin{array}{llllllll}
+1 & +1 & -1 & -1 & -1 & -1 & +1 & +1
\end{array}\right] \text {; }
$$

and then both signals are added. One aggregate signal is transmitted through the air medium. To simulate the noise and interference during the transmission through the airy channel a random sequence of $1 \mathrm{~s}$ and $0 \mathrm{~s}$ is produced and added to the transmitted signal. The noise is generated by using the following Matlab command:

$$
\text { noise }=\operatorname{randsrc}\left(1,1 \text { en_trans },\left[0,1 ; p_{-} 0, p_{-} 1\right]\right) \text {; }
$$


where $p_{-} 0$ is the probability of occurrence of 0 while $p_{-} 1$ is the probability of occurrence of 1 . The variable 'len_trans' is the length of the transmitting aggregated signal. In the simulation, $p_{-} 0$ and $p_{-} 1$ are set to $98 \%$ and $2 \%$ respectively. The $2 \%$ occurrence of ones in the noise gives us approximately 60,000 errors that are to be corrected.

At the receiver side, the received corrupted signal is first multiplied with both chip sequences, $C_{2} A$ and $C_{-} B$, in order to extract the signal meant for the Channel $A$ as well as for the Channel $\mathrm{B}$. The error correction is performed as well as per discussion given in Chapter 5. If not all errors are detected and corrected during this stage, the remaining ones will be corrected during the channel decoding stage (Linear Block Code decoder for the Channel $A$ and Convolutional Code decoder for the Channel B). After separating the received signal to two Channels $A$ and Channel $B$ signal, each of these signals is XOR-ed with the pseudo generated noise used at the transmitter end (both pseudo generated sequences are known in advance to the receiver). At this point, we have two received signals that represent the received speech and still image data. The decoding principles and capabilities of Linear Block Code and Convolutional Code algorithm are presented and discussed in Chapter 4 and will not be discussed here again. A reader is encouraged to read Chapter 4 , in order to understand the decoding principles of the both channel decoders. At the source decoding stage, the speech and image data are reconstructed using Lempel-Ziv-Welch and Huffman Code tables to the ir analog equivalents and the both signals are displayed. Once again, the coding tables are known to the decoder in advance for both techniques. The exception is the adaptive Huffman tree decoding, where the encoder and decoder only share the knowledge about the number of symbols in the alphabet.

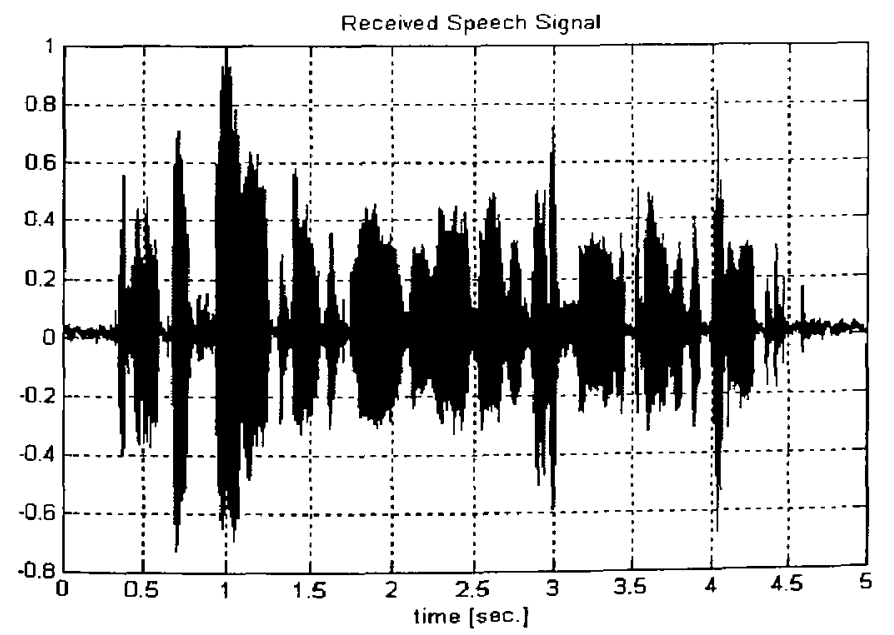

Figure 23: Received Speech Data.

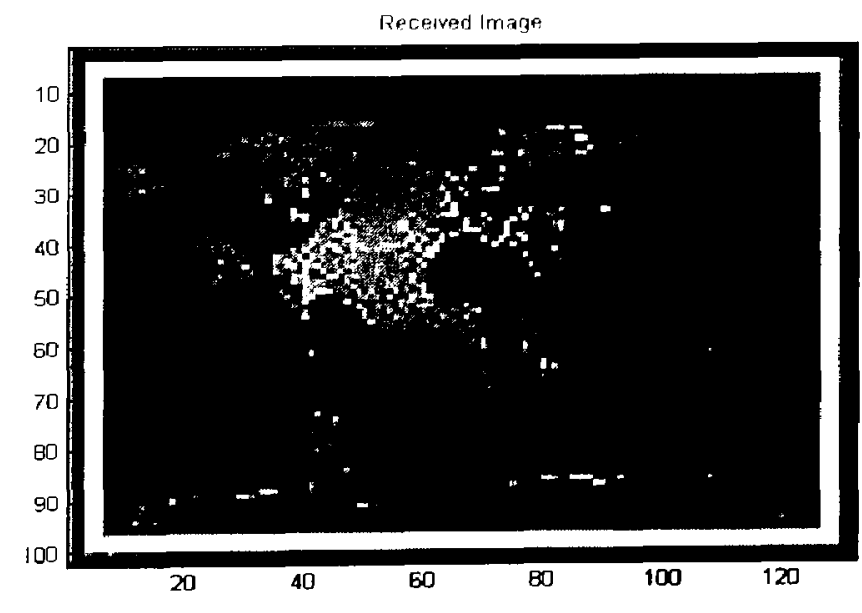

Figure 24: Received Image. 
As the very last step on Channel $A$, the received digital speech signal is converted back to its analog representation and is played in Matlab using the 'wavplay' command. The received speech signal plotted in the time domain is shown in Figure 23. The received image is shown in Figure 24.

\section{Conclusion}

The project presents a source-channel codec for a WCDMA based multimedia system. The system consists from two Channels, A and B, where the Channel A is reserved for transmitting/receiving a speech signal while the Channel $B$ transmits/receives a still image data. On the transmitter end, the Channel A has the following stages: formatting (sampling, quantizing, and PCM), source encoding (Lempel-Ziv-Welch Encoder), channel encoding (Linear Block Code Encoder), and WCDMA medium access control (DSSS plus Chip Sequencing) stage. On the other side, the Channel B has these stages at the transmitter: formatting (ASCII), source encoding (Huffman Code Encoder), channel encoding (Convolutional Code Encoder), and WCDMA medium access control (DSSS plus Chip Sequencing) stage.

At the receiver end, firstly, the received aggregate signal is divided into two signals; the signal meant for the Channel $\mathrm{A}$ and the signal meant for the Channel B. The signal meant for the Channel A (speech signal) goes through the following stages: channel decoder (Linear Block Code Decoder), source decoder (Lempel-Ziv-Welch Decoder), and digital to analog conversion. The signal that is selected for the Channel $\mathrm{B}$ goes through: channel decoder (Convolutional Code Decoder) and source decoder (Huffman Code Decoder).

Throughout the project I have faced with quite a few challenges in implementing the codec. The biggest one was the time of the execution. Matlab as a language of choice for this project has not had the needed speed capabilities. Some implementations of the algorithms such as Lempel-Ziv-Welch encoding and Convolutional Code decoding have taken way too much time, approximately 2 days for each simulation! An interested student who would have patience and willingness to update this project should choose a programming language that is based on the concurrent type of code execution instead of using a language based on sequential type of code execution. The sequential languages such as Matlab execute a code in a line by line fashion, while the concurrent languages such as VHDL execute several lines of code at the same time (the analogy would be as if a computer had several processors that are capable of executing the lines at the same time). That's why a concurrent language would be more appropriate for this type of project. The ideal solution would be to implement this project in hardware. 
Another recommendation to improve this project is not to use Lempel-Ziv-Welch algorithm for a speech signal. Besides the fact that the execution of its simulation is too slow, another problem is that a Lempel-Ziv-Welch code word can contain 12 bits only. If a binary data sequence is too long, as they usually are, there is a need to create more than one table which takes additional space in the memory. The better solution would be to use adaptive Huffman Code algorithm to compress a speech signal.

The error detecting and correcting capabilities are another thing that could be improved. A Linear Block Code that would detect and correct as many bit error patterns as possible is needed (in the real world $(127,92)$ linear block code is used). As stated earlier in this concluding section, a programming language that can execute the code faster must be used (e.g. $\mathrm{C}++$ ) to improve the speed of detecting and correcting processes.

To sum up, the project shows all necessary stages that are needed for transmitting and receiving multimedia signals through a digital system. Complex multimedia systems may have more stages than the system shown in this project does, but the stages presented and implemented in this project make a foundation for all digital systems and absolutely can not be excluded as a part of their implementations. 


\section{References:}

[1] F. G. Stremler, Introduction to Communication Systems., Addidson - Wesley Series in Electrical Engineering, 1997.

[2] B. Sklar, Digital Communications: Fundamentals and Applications., PTR Prentice Hall, 1998.

[3] J. G. Proakis, Digital Communications., McGraw Hill, 2001.

[4] T. S. Rappaport, Wireless Communications: Principles and Practice., Prentice Hall, 2002.

[5] A. L. Garcia, I. Widjaja, Communication Networks: Fundamental Concepts and Key Architectures., McGraw Hill, 2003.

[6] M. Abramovici, M. Bauer, Digital Systems Testing and Testable Design., Wiley Inter Science, 1990

[7] W. W. Wu, Elements of Digital Satellite Communication., Computer Science Press, 1995.

[8] R. J. Sluyter, "Digitalization of Speech" in Philips Tec. Rev., vol. 41. pp. 201-221.

[9] N. S. Jayant, Digital Coding of Waveforms., Prentice Hall, 2000.

[10] T. J. Lynch, "Data Compression Techniques and Applications", in Lifetime Learning Publications, Belmont, Calif., 1985.

[11] J. M. Tribolet, "Speech Coding", in IEEE Transactions on Communications, vol. 27, no. 4, April 1979, pp. 710-737.

[12] W. W. Peterson, Error Correcting Codes., MIT Press, 1972.

[13] G. C. Clark, Error Correcting Coding for Digital Communications., Plenum Press, 1981.

[14] R. G. Gallager, Information Theory and Reliable Communication., John Wiley \& Sons, 1968.

[15] The Student Edition of MATLAB, The User's Guide., The Math Works Inc., 1995

[16] A. J. Viterbi, CDMA: Principles of Spread Spectrum Communication., Addison - Wesley Readings. 
[17] "Coding Techniques for Digital Communications," class notes for EE8110, Department of Electrical and Computer Engineering, Ryerson University, Winter 2005. 
Appendix A:

Matlab Simulation Files 


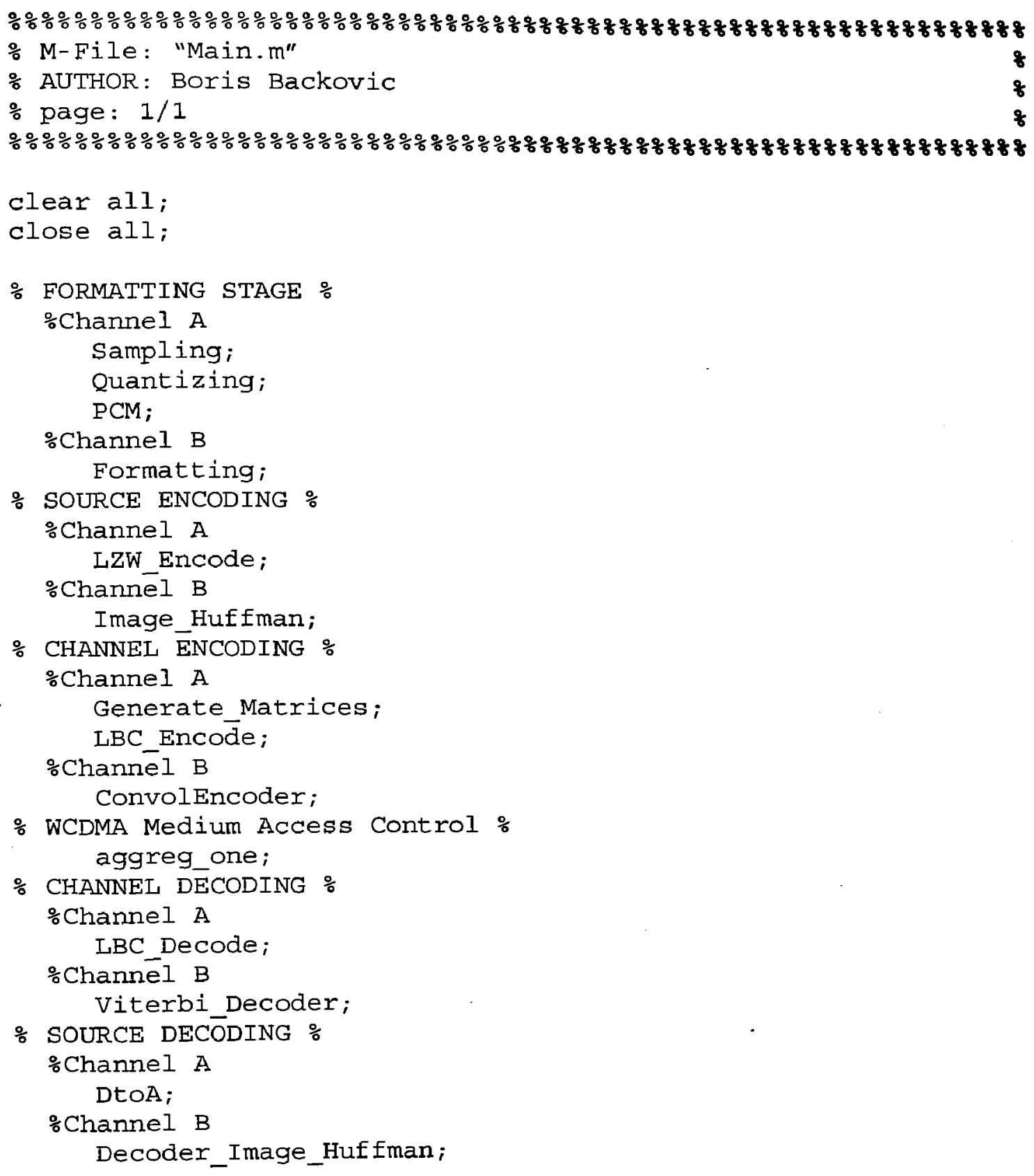




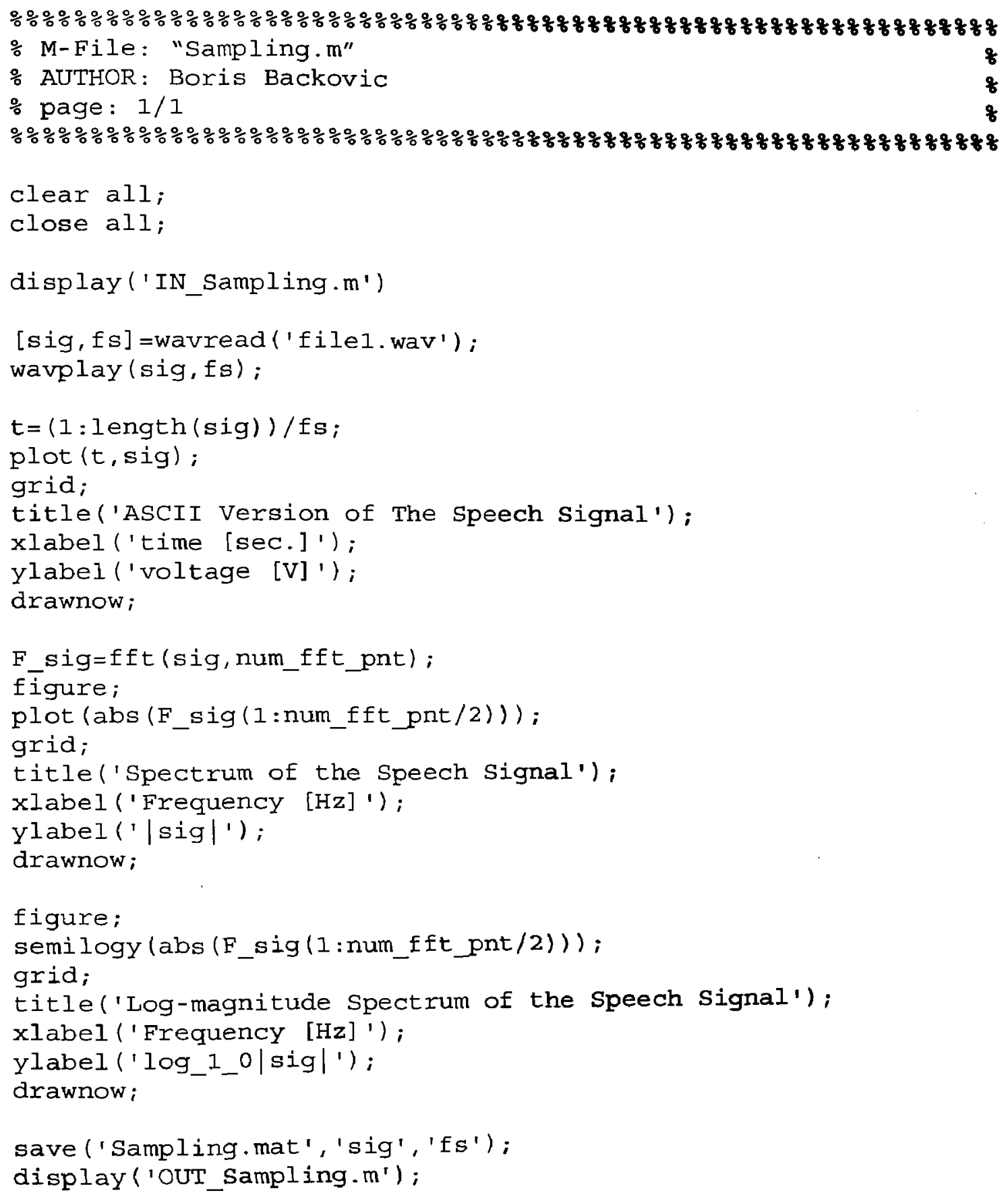




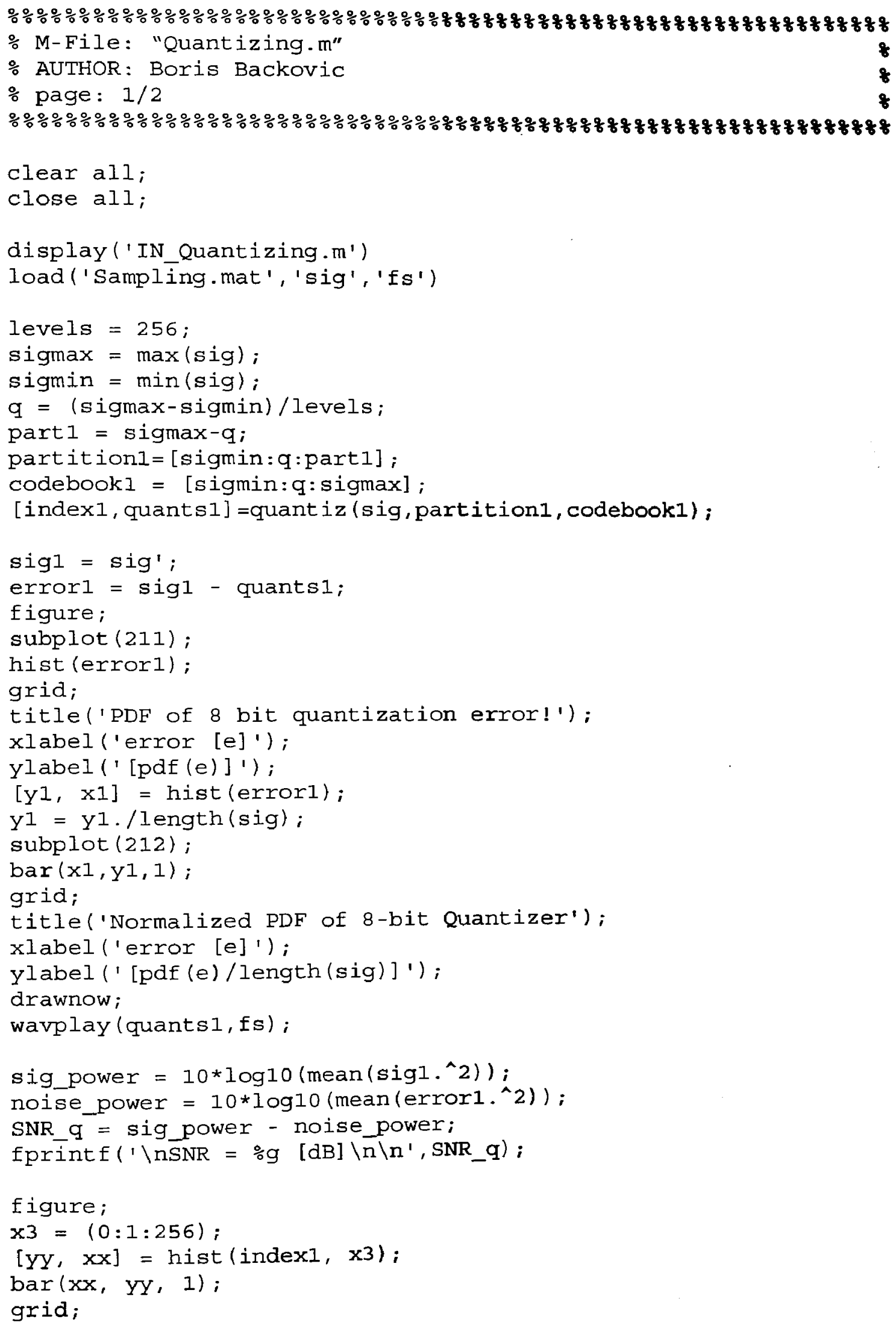




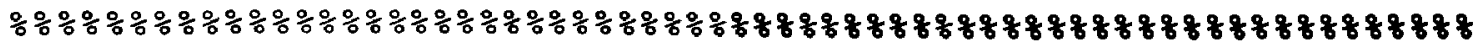

\% M-File: "Quantizing.m"

\% AUTHOR: Boris Backovic

\% page: $2 / 2$

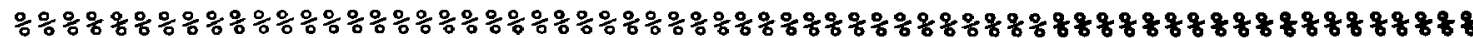

title('Usage of Quantizer Levels');

$x$ label ('qunatization levels');

ylabel ('frequency of occurrence');

save ('Quantizing.mat ', 'index1', 'quants1')

display ('OUT_Quantizing.m') 


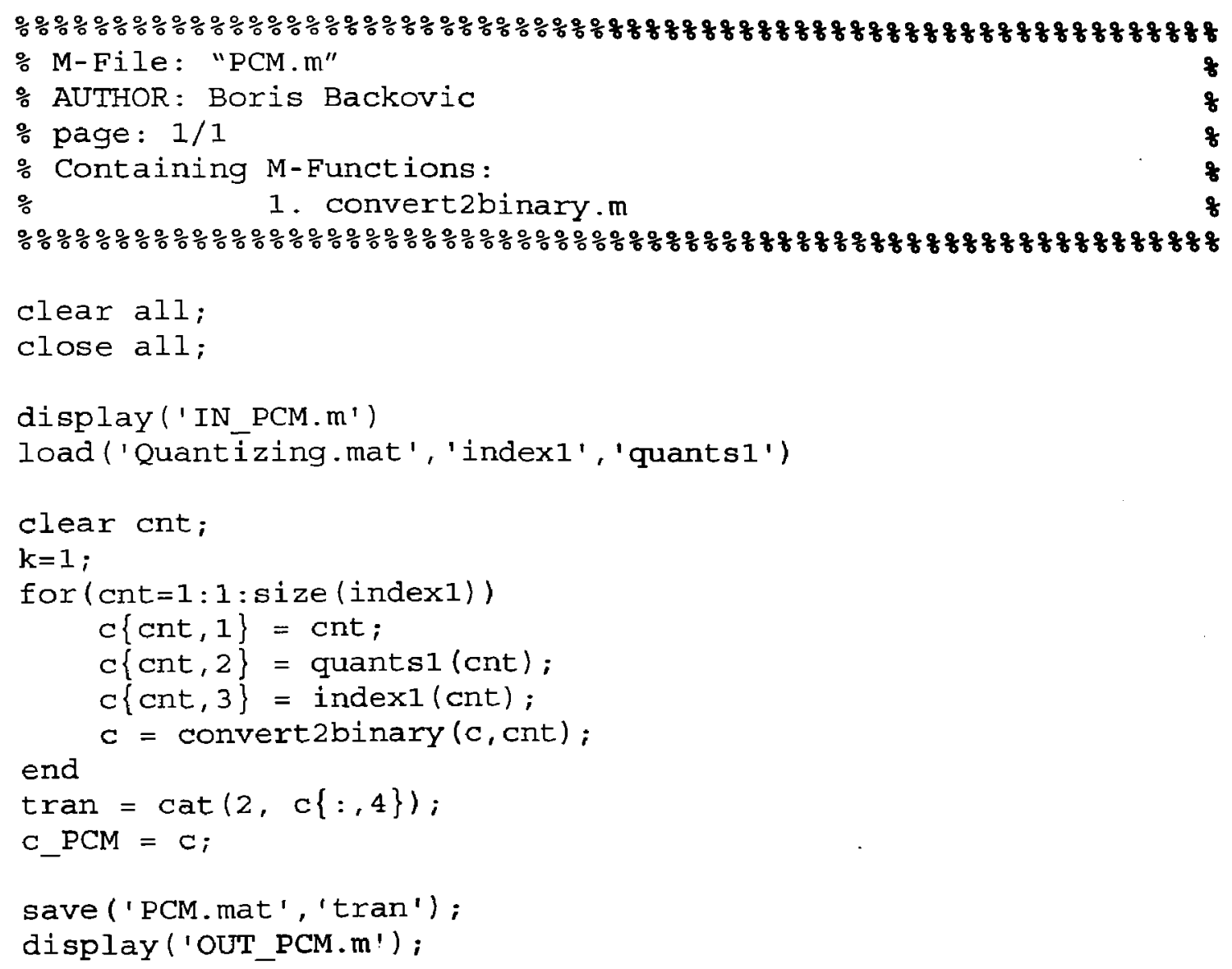




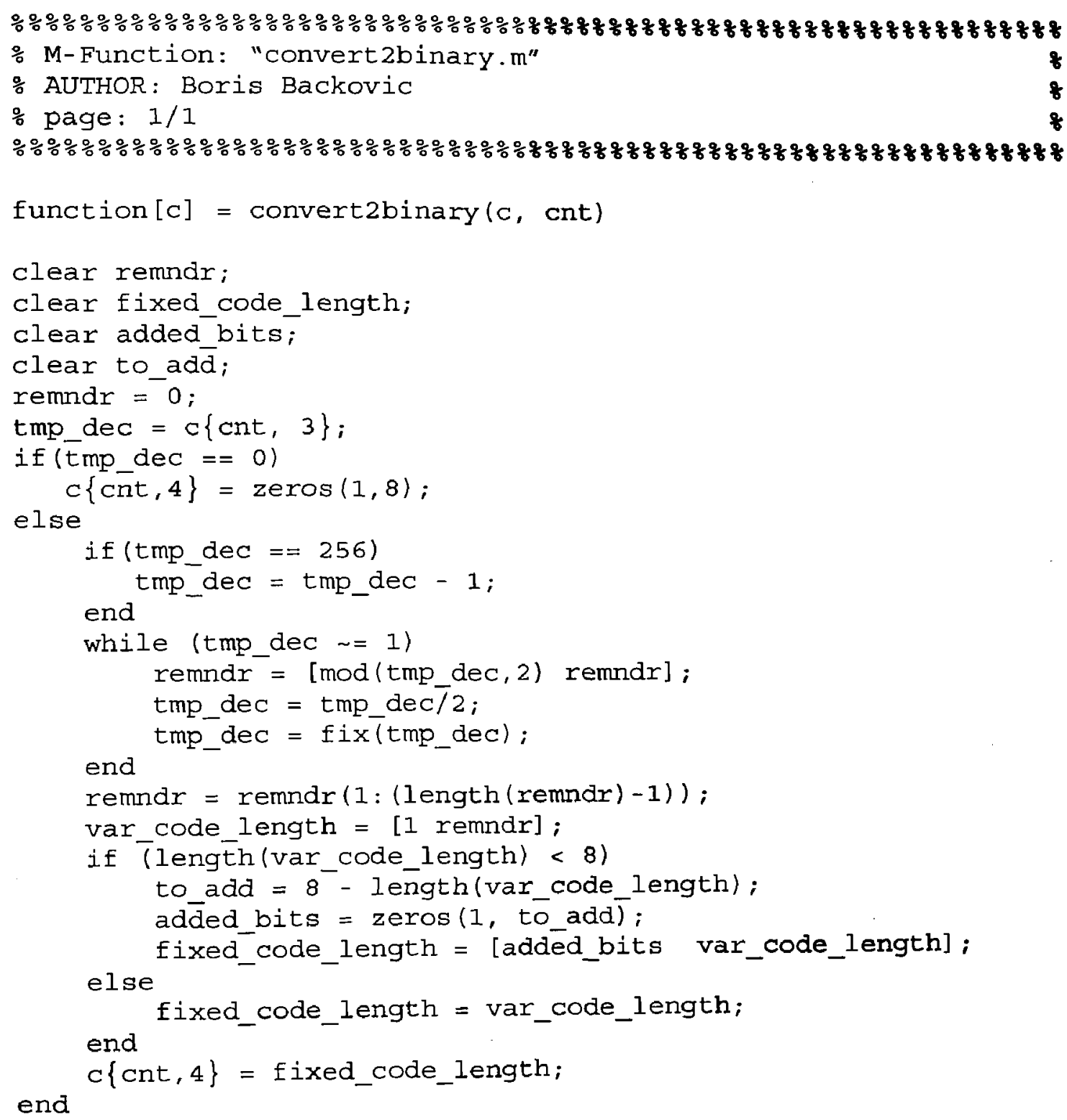




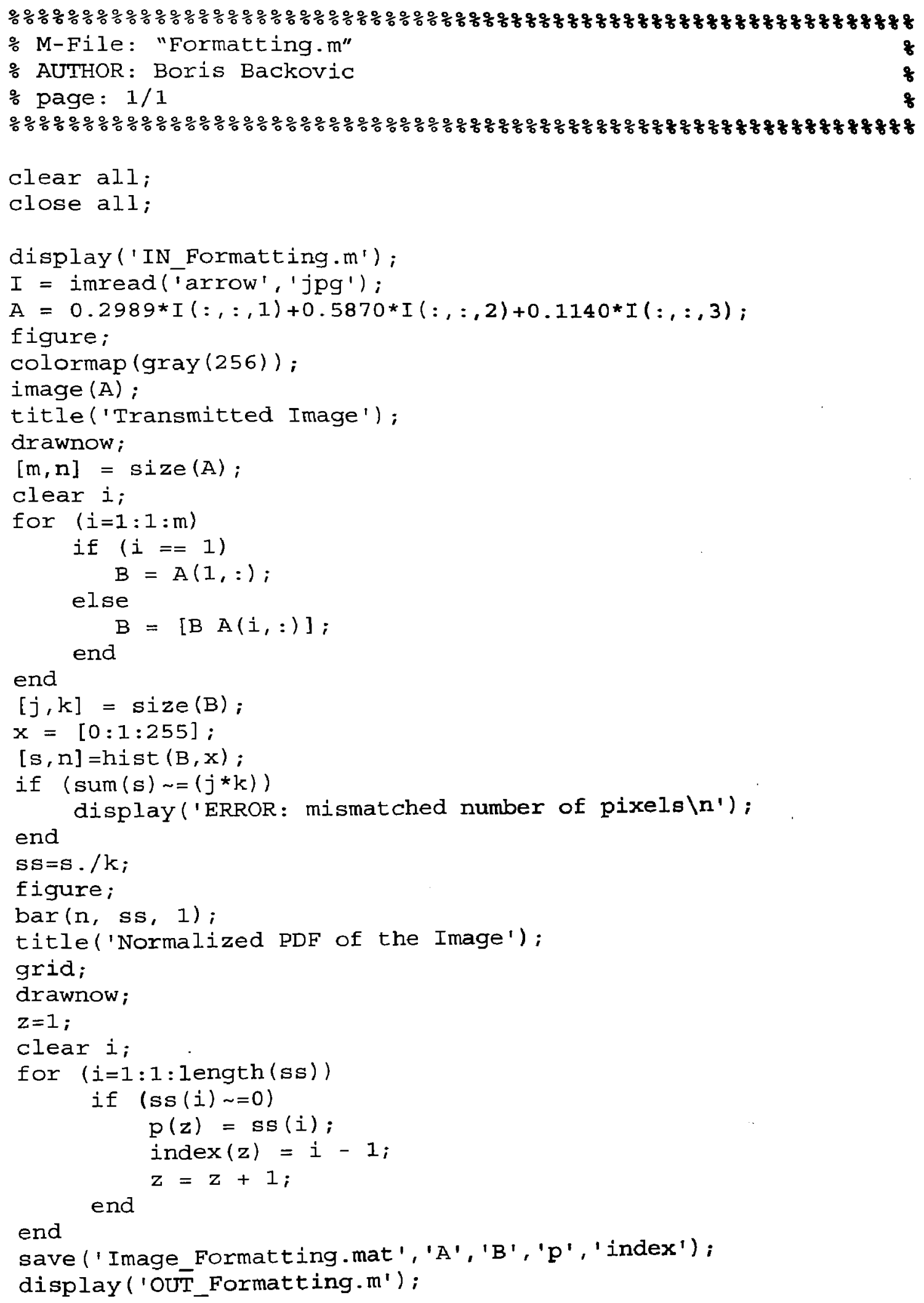




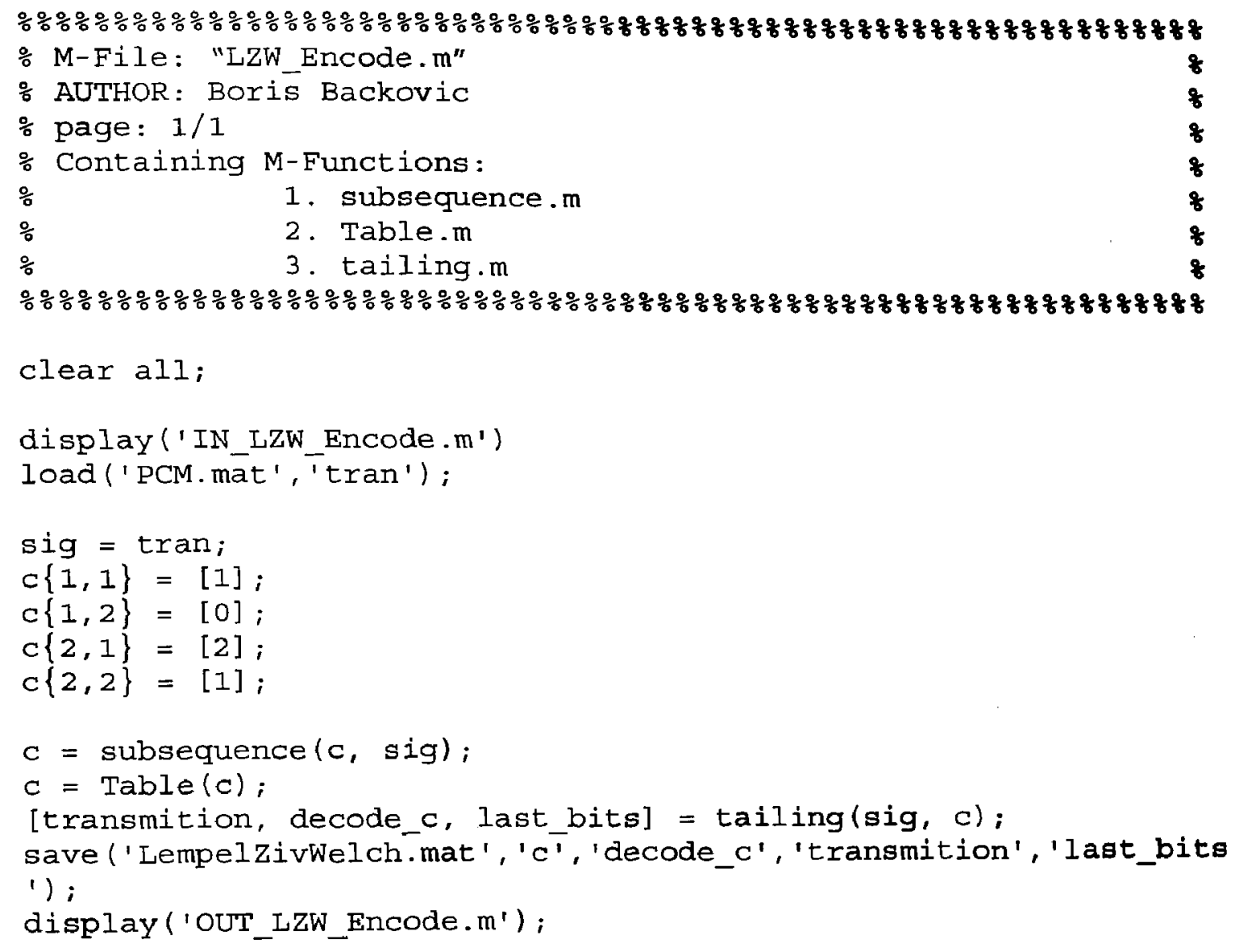




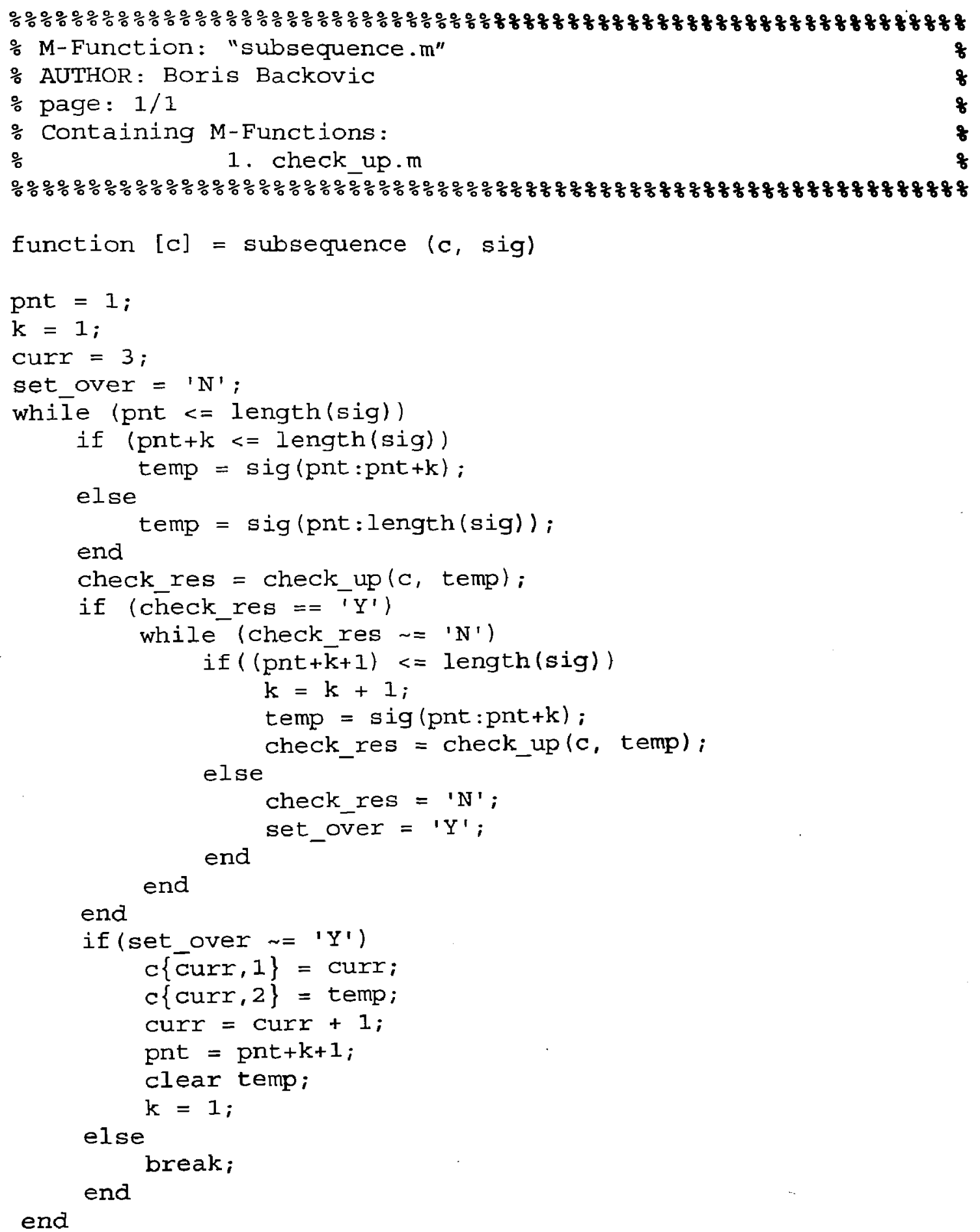




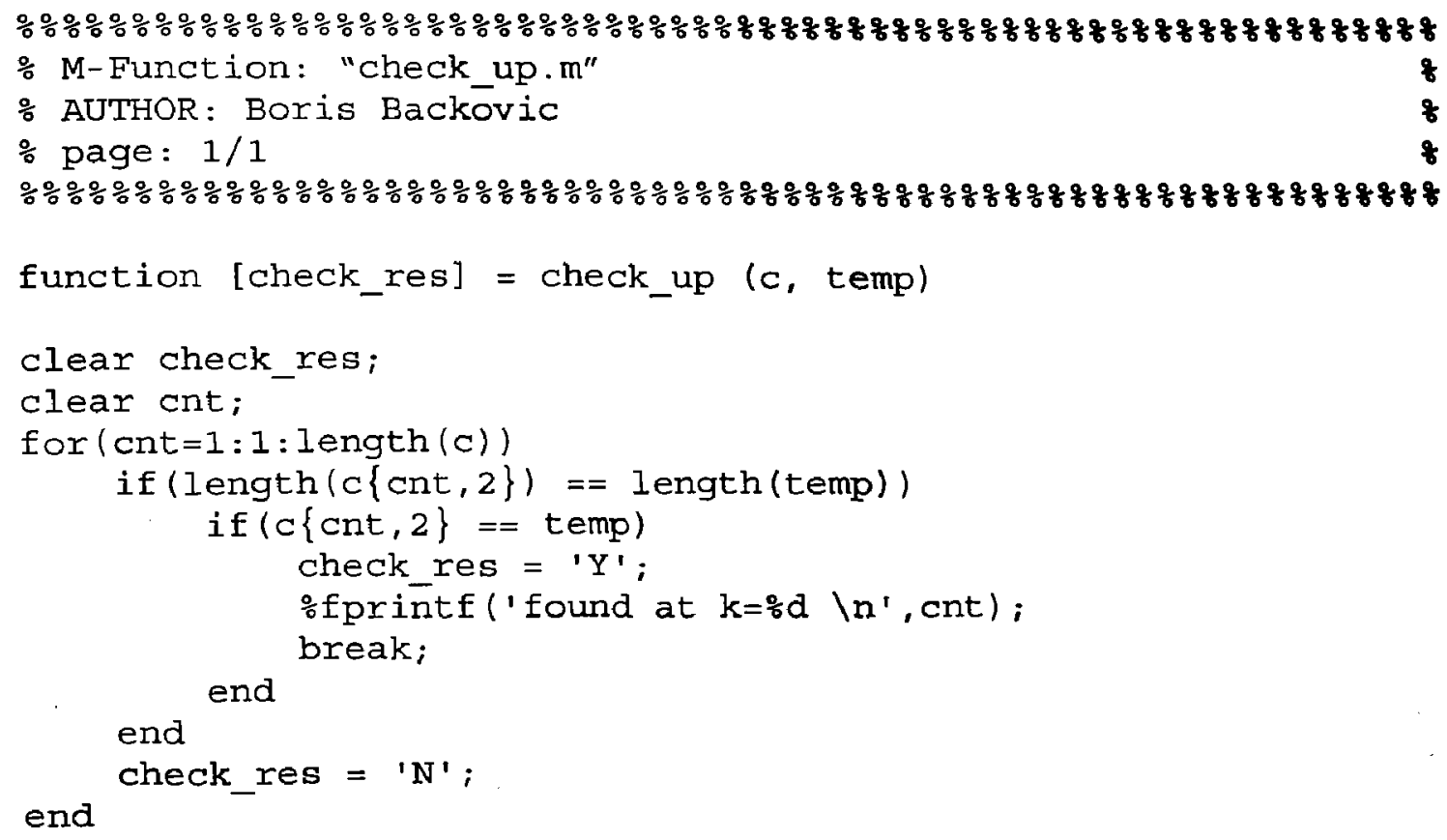




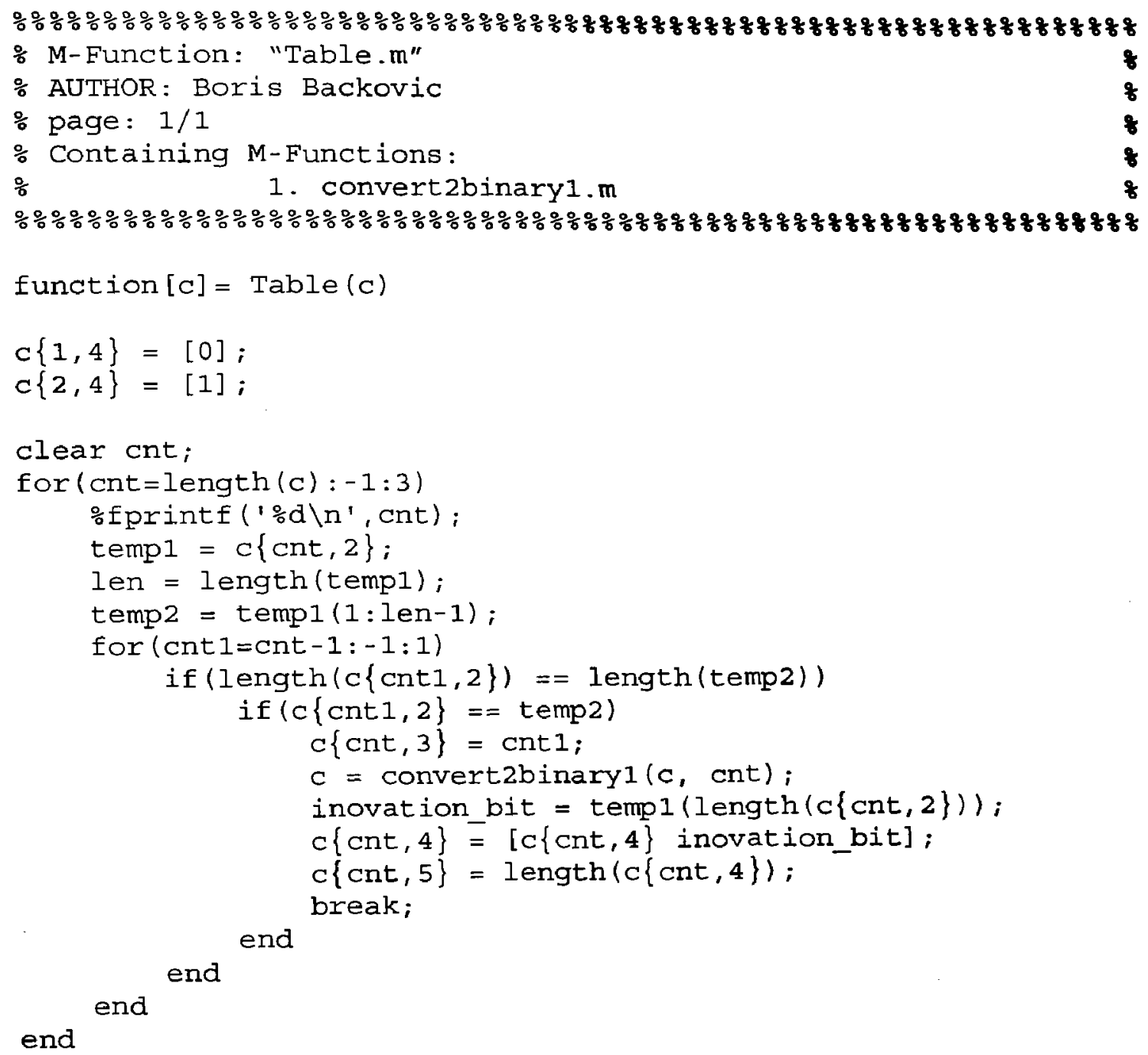




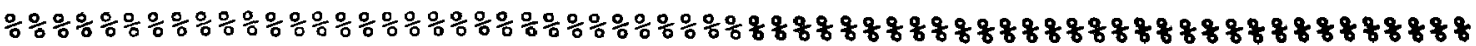

$\div$ M-Function: "converting2binaryl.m"

: AUTHOR: Boris Backovic

\% page: 1/1 1

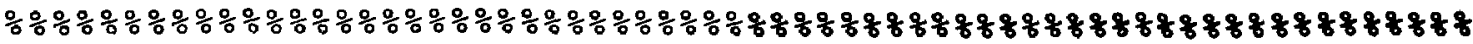

function $[c]=$ convert 2 binaryl $(c, c n t)$

clear remndr;

clear fixed_code_length;

clear added_bits;

clear to_ad, ;

remind $=0$;

tmp_dec $=c\{c n t, 3\}$;

if (tmp_dec $==0$ )

$c\{\overline{c n t}, 4\}=0$;

else

while (tmp_dec $\sim=1$ )

remndr $=\left[\bmod \left(t m p \_d e c, 2\right)\right.$ remndr] ;

tmp_dec $=t m p \_d e c / 2$;

end

tmp_dec $=f i x\left(t m p \_d e c\right)$;

remndr $=\operatorname{remndr}(1:($ length $($ remndr $)-1))$;

var code length $=$ [1 remndr];

end

$c\{\overrightarrow{c n t}, 4\}=$ var_code_length; 


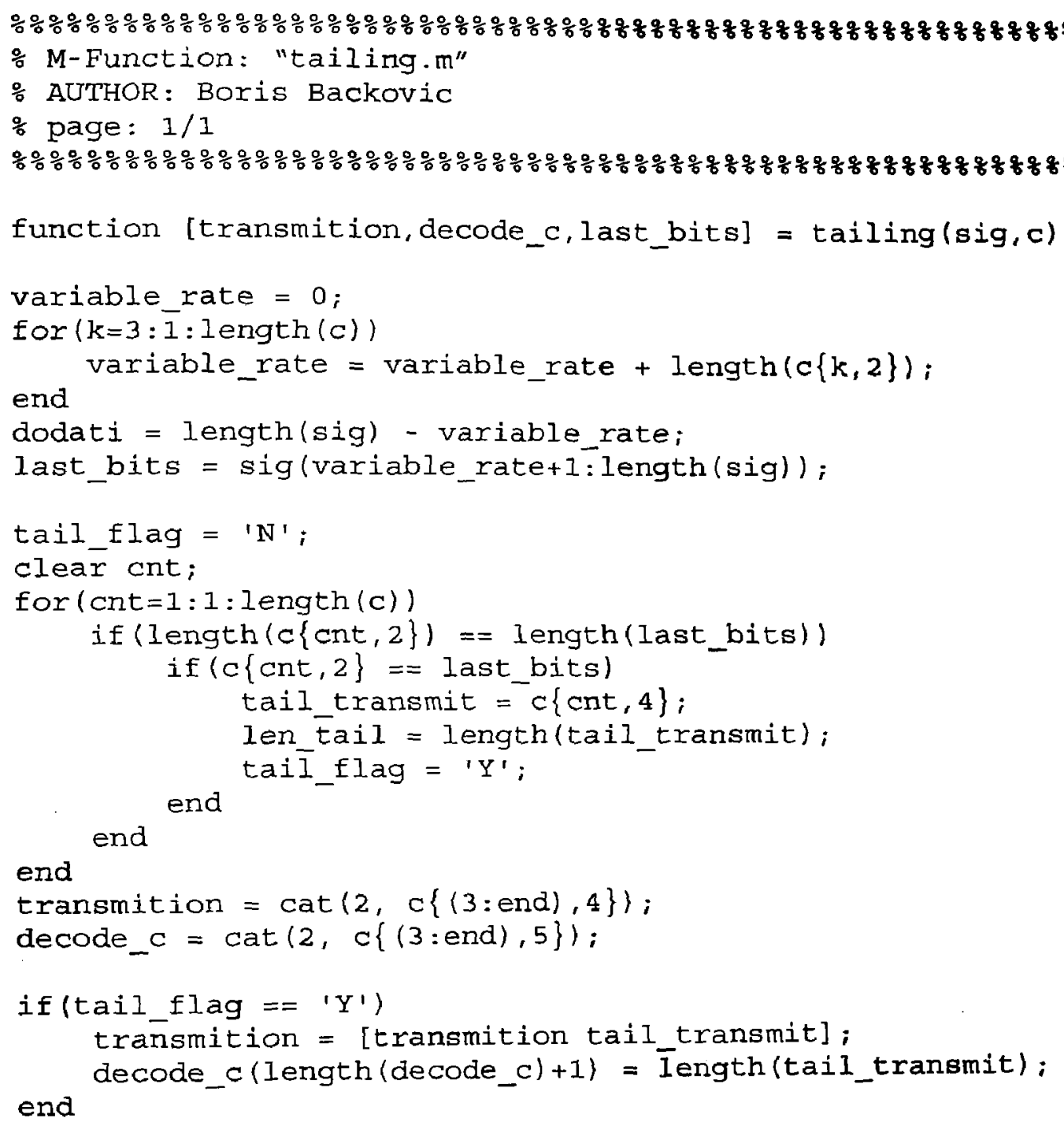




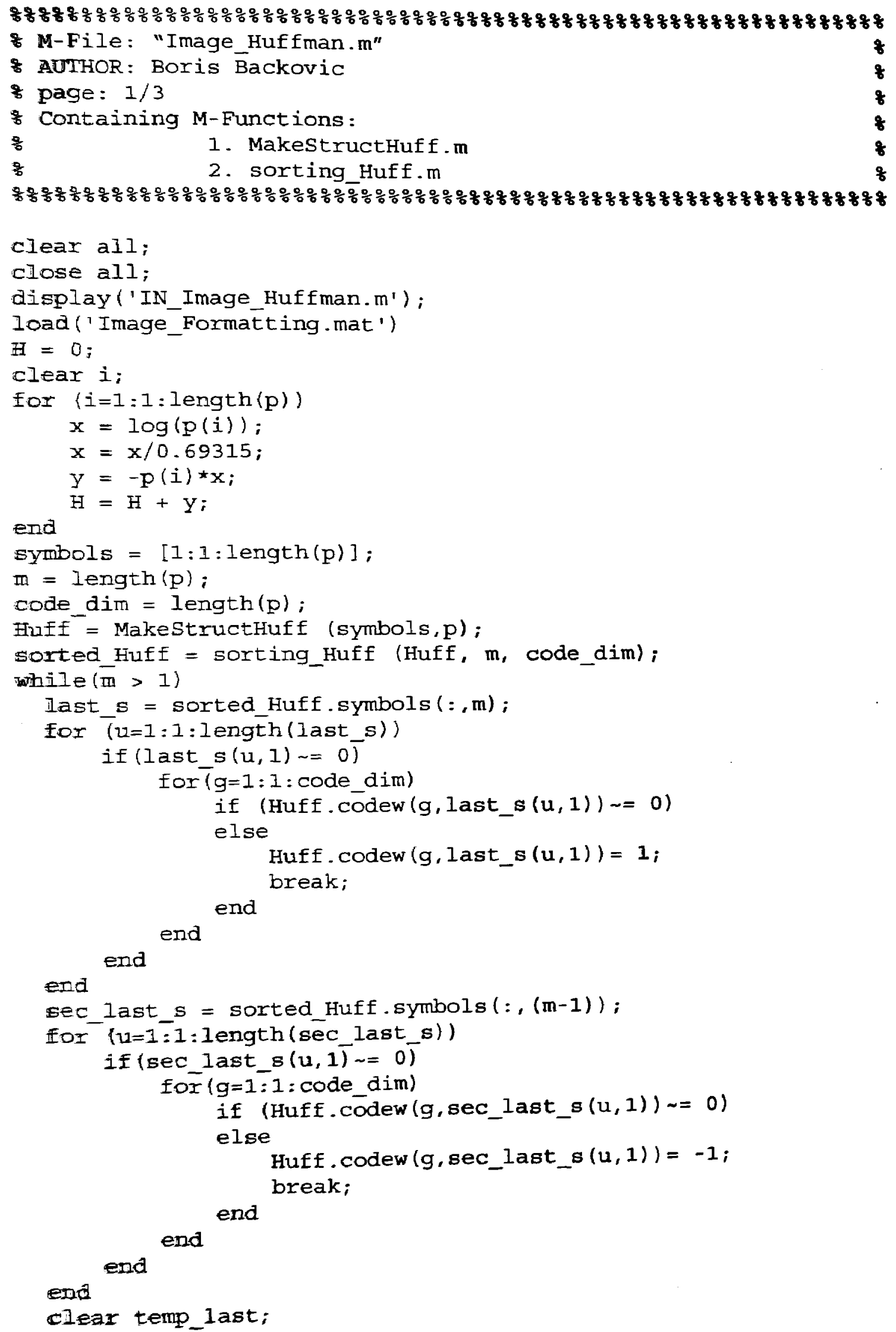




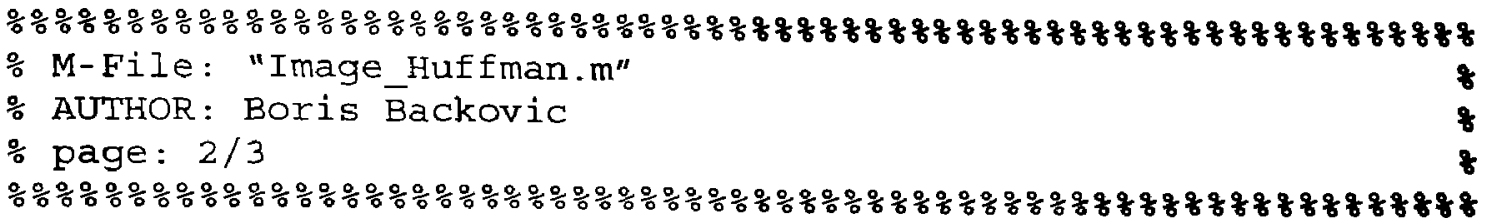

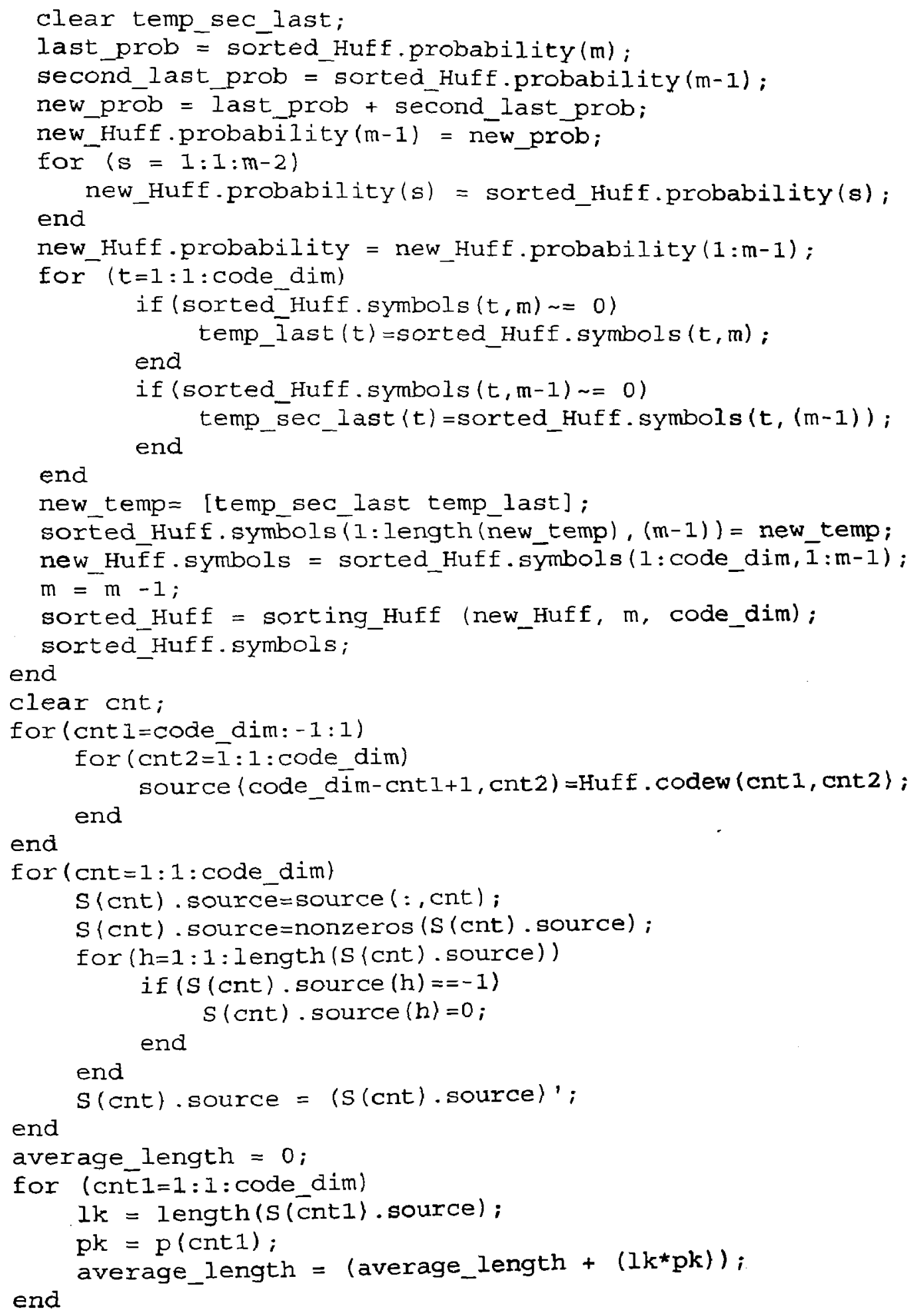




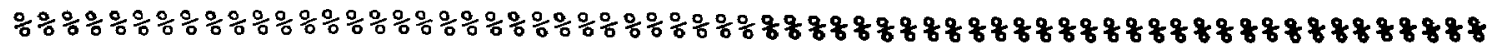

\% M-File: "Image_Huffman.m"

\% AUTHOR: Boris Backovic

$\%$ page: $3 / 3$

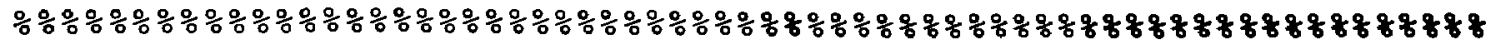

clear i;

clear $\mathrm{k}$;

for $(k=1: 1:$ length $(B))$

for ( $i=1: 1:$ length (index))

if (index $(i)==B(k)$ )

if $(k==1)$

bit_stream $=S(i)$. source;

else

length_of_each_letter = length(s(i).source);

bit_stream $=$ [bit_stream $s(i)$.source];

length_of_each_letter $=$ [length_of_each_letter

length (S (i). source)]; end

end

end

end

save ('Image_Huffman.mat', 'bit_stream', 'length_of_each_letter', 'S' , 'index', 'A $\bar{\top})$;

display ('OUT_Image_Huffman.m'); 


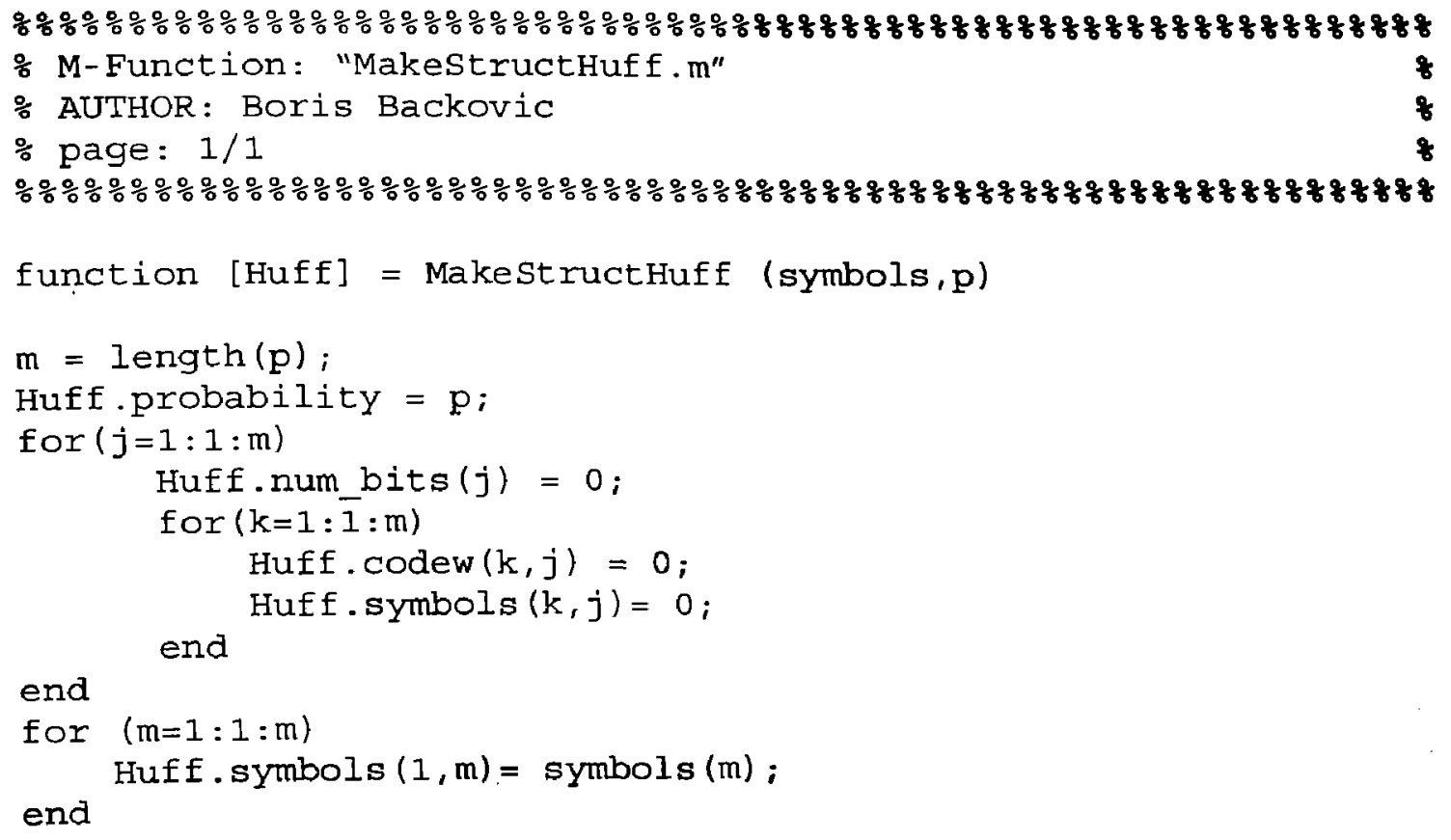




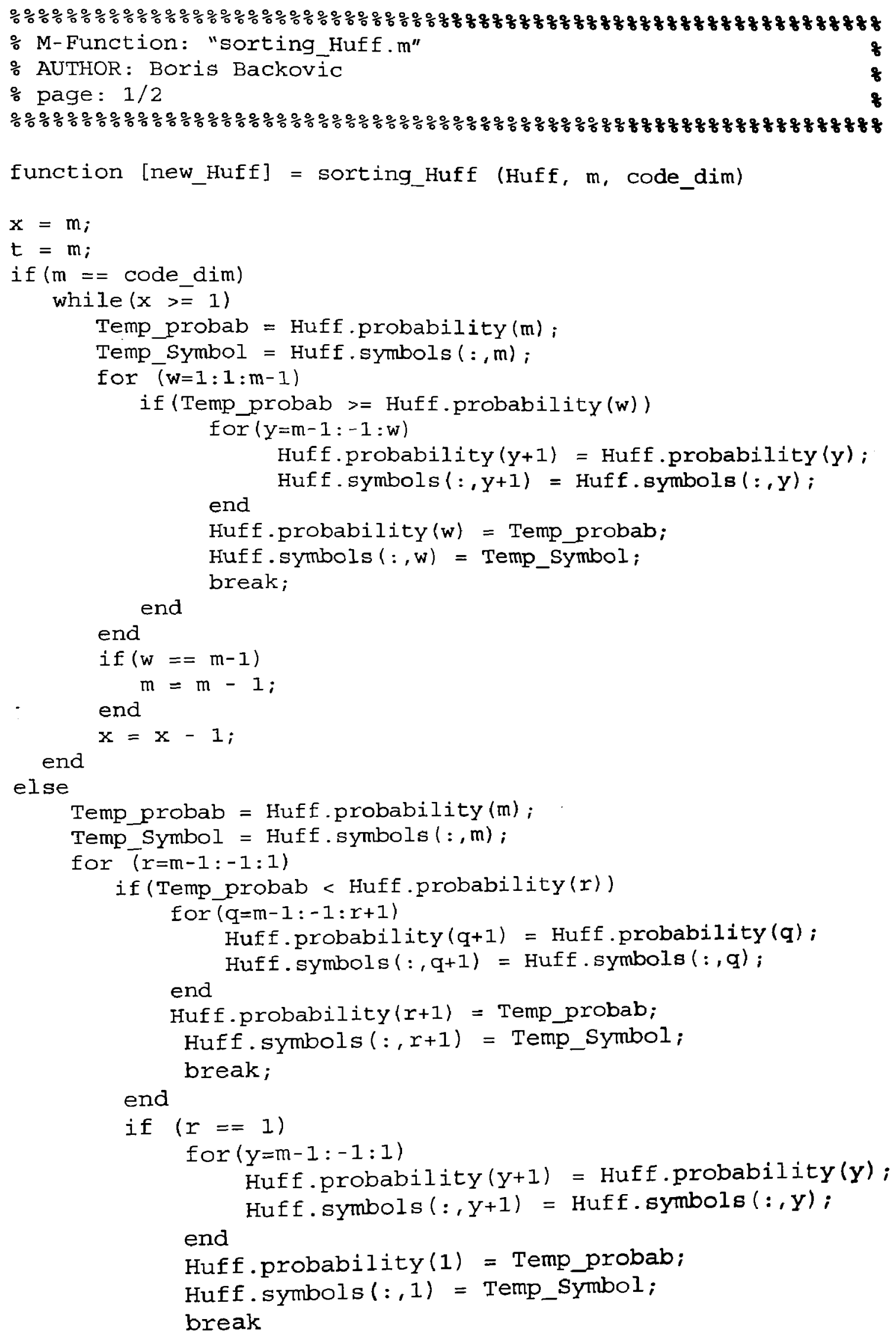




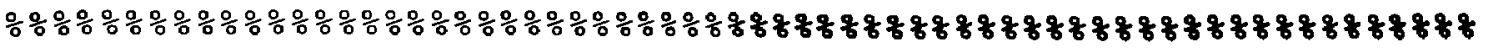

응 $\mathrm{M}$-Function: "sorting_Huff.m"

: AUTHOR: Boris Backovic of

\% page: $2 / 2$ q

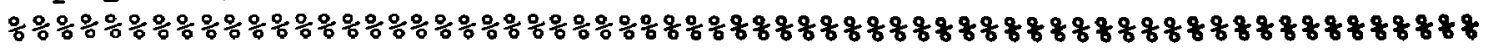

end

end

end

new_Huff = Huff; 


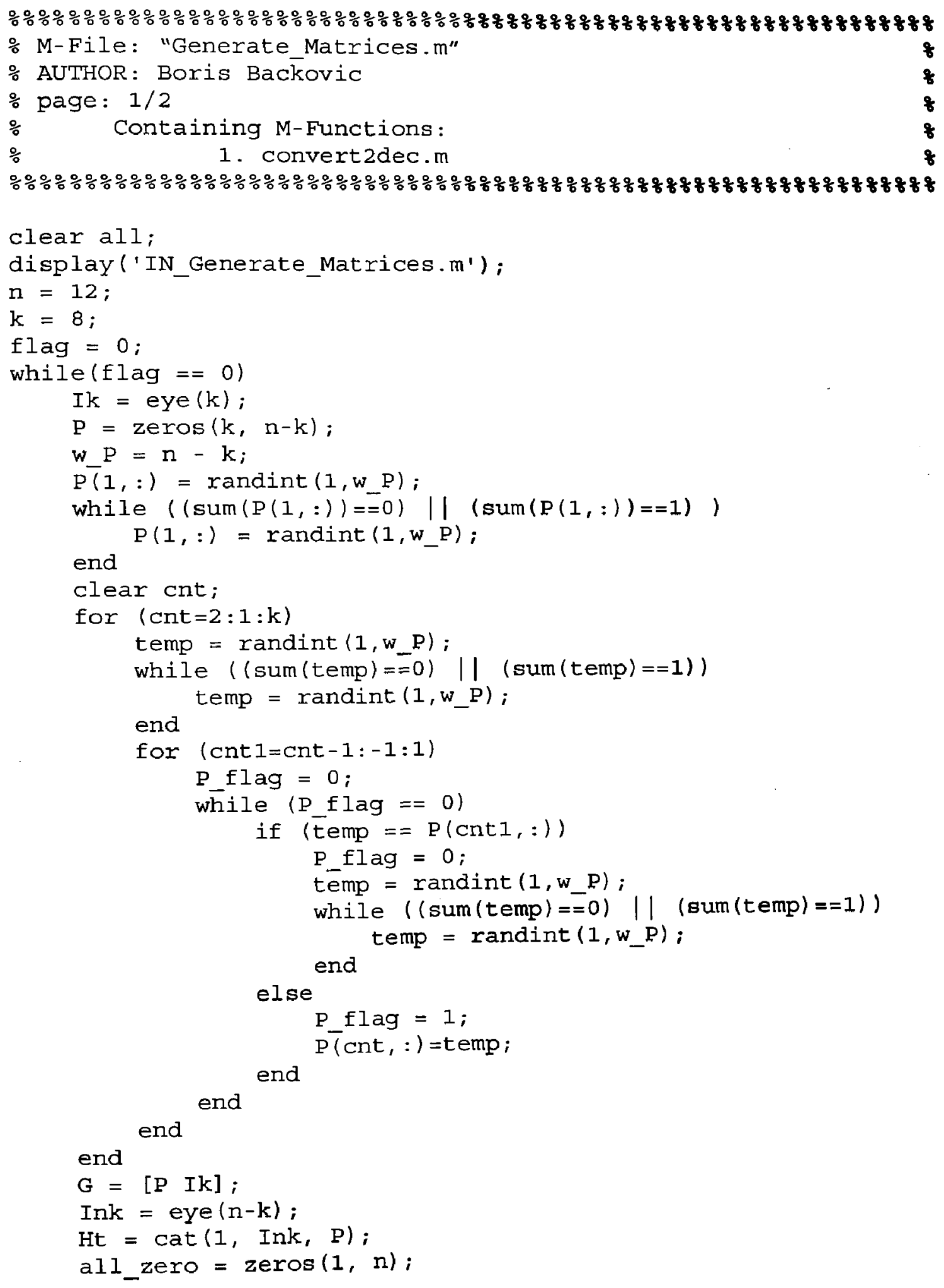




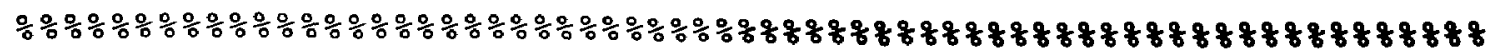

․ M-File: "Generate_Matrices.m" \&

\% AUTHOR: Boris Backovic f

\% page: $2 / 2$

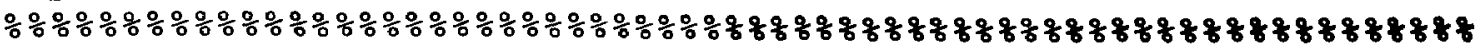

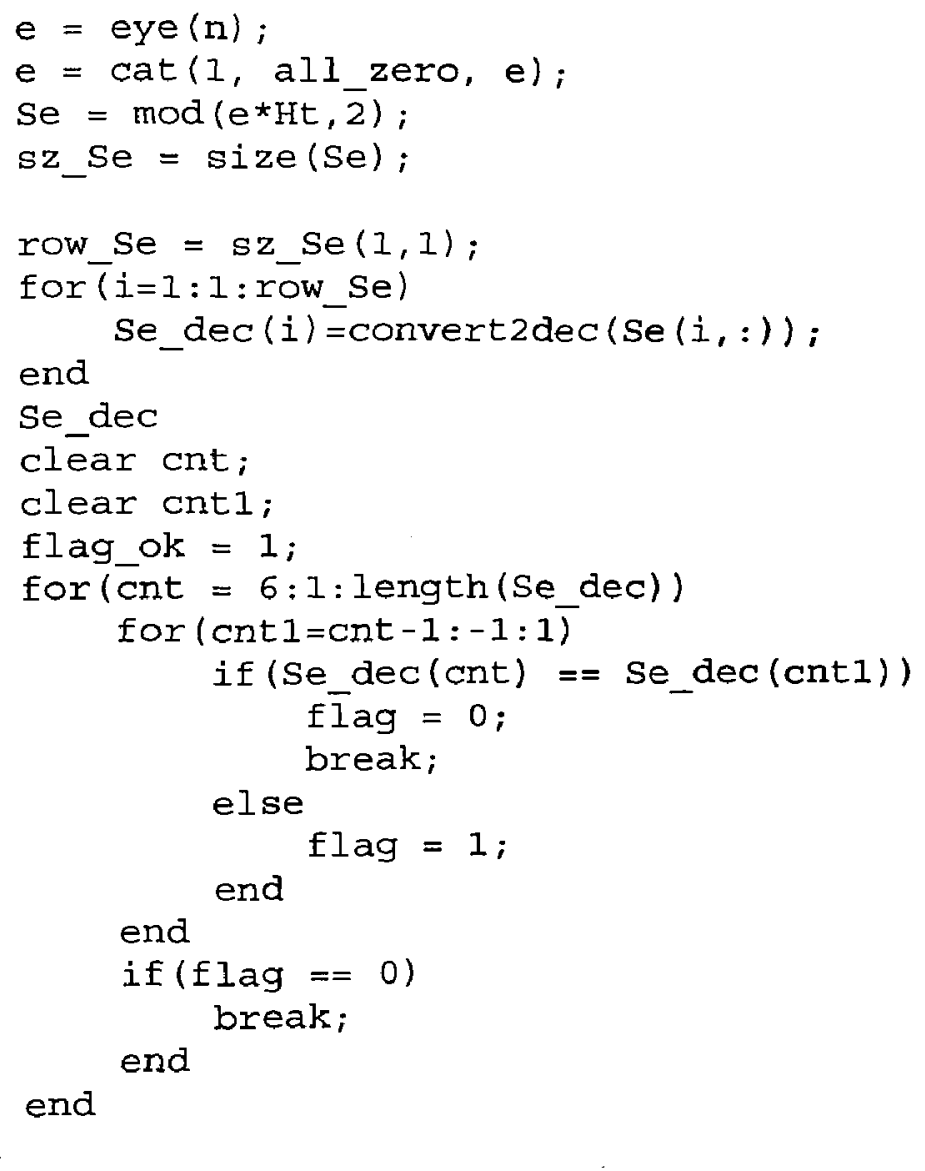

save ('Generate_Matrices.mat', 'n', 'k', 'row_Se', 'Ik', 'Ink', 'p', 'G', 'Ht', 'e', 'Se');

display ('OUT_Generate_Matrices.m'); 


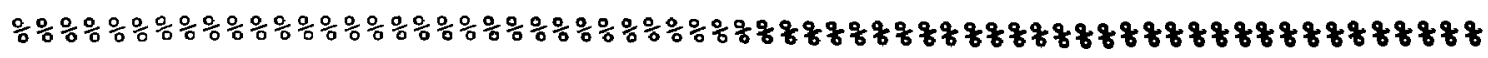

\% M-Function: "convert 2 dec.m"

\% AUTHOR: Boris Backovic

\% page: $1 / 1$

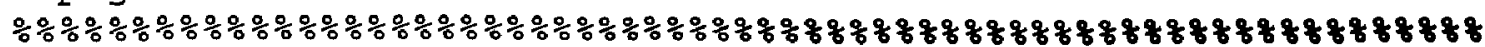

function [d] = convert $2 \mathrm{dec}(\mathrm{b})$

$\mathrm{d}=0$;

$\mathrm{k}=$ length (b) ;

for $(\mathrm{cnt}=1: 1: k)$

$d=\left(b(c n t) *\left(2^{\wedge}(k-c n t)\right)\right)+d ;$

end 


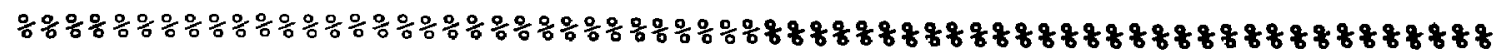

\% M-File: "LBC_Encode.m"

\% AUTHOR: Boris Backovic

page: $1 / 1$

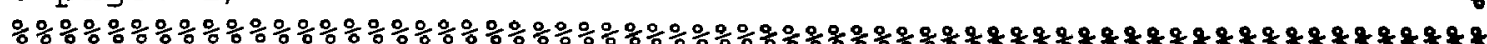

clear all;

display ('IN_LBC_Encode.m')

load ('Generāte_Matrices.mat', 'n', 'k', 'G') ;

load ('LempelzivWelch.mat', 'transmition');

$m=$ transmition;

$C C=0 ;$

while (rem(length $(\mathrm{m}), 8) \sim=0$ )

$\mathrm{m}=[\mathrm{m} 0] ;$

end

$\mathrm{CC}=\mathrm{CC}+1 ;$

pnt $=1_{i}$

indx $=1$;

while (pnt < length (m))

message $\{$ indx, 1$\}=m$ (pnt:pnt $+k-1) ;$

$\mathrm{U}\{\mathrm{indx}, 1\}=\bmod (\operatorname{mes} s a g e\{$ ind $x, 1\} * \mathrm{G}, 2)$;

pnt $=$ pnt $+\mathrm{k}$;

end

ind $x=$ ind $x+1$;

transmit $=\operatorname{cat}(2, \mathrm{U}\{:, 1\})$;

save ('BLC_Encode.mat', 'transmit', 'U', 'message', 'm' , 'CC'); display('OUTT_LBC_Encode.m') 


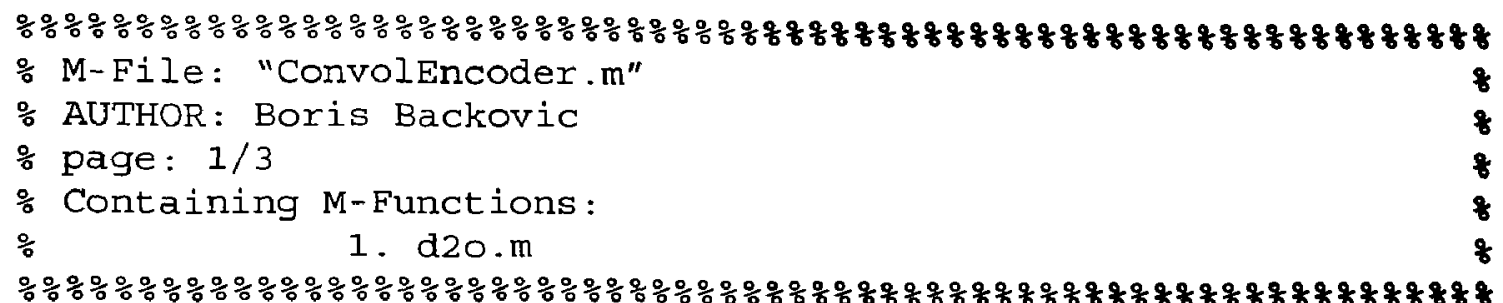

clear all;

display ('IN ConvolEncoder.m');

load ('Image_Huffman.mat', 'bit_stream');

num_output_per_bit $=2$;

generator $=\left\{\left[\begin{array}{llllll}\overline{1} & 1 & 1 ; & 1 & 0 & 1\end{array}\right]\right.$,

$\left[\begin{array}{llll:llll}1 & 1 & 0 & 1 ; & 1 & 1 & 1 & 0\end{array}\right]$,

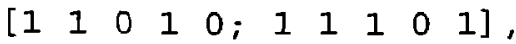

$\left[\begin{array}{llllllllllll}1 & 1 & 0 & 1 & 0 & 1 ; & 1 & 1 & 1 & 0 & 1 & 1\end{array}\right]$,

$\left[\begin{array}{llllllllllll}1 & 1 & 0 & 1 & 0 & 1 ; & 1 & 1 & 0 & 1 & 0 & 1\end{array}\right]$,

$\left[\begin{array}{lllllllllllllll}1 & 1 & 0 & 1 & 1 & 1 & 0 & ; & 1 & 1 & 1 & 0 & 0 & 1 & 1\end{array}\right]$,

$\left[\begin{array}{llllllllllllllllll}1 & 1 & 0 & 1 & 1 & 1 & 0 & 0 & 1 ; & 1 & 1 & 1 & 0 & 0 & 1 & 1 & 0 & 1\end{array}\right]$,

$\left[\begin{array}{llllllllllllllllllll}1 & 1 & 0 & 1 & 1 & 1 & 0 & 0 & 1 & 0 ; & 1 & 1 & 1 & 0 & 0 & 1 & 1 & 0 & 0 & 1\end{array}\right]$;

$m=2 ;$

$M=m+2$;

look up $=$ generator $\{\mathrm{m},:\}$;

gen_v 1 l $=100 k$ up $(1,:)$;

gen_v2 $=$ look_up $(2,:)$;

clear $i$;

for $(i=1: 1: M)$

impuls_res $=$ zeros $(\mathrm{M})$;

imp_in $=$ impuls_res $(1,:)$;

imp_in $(i)=1$;

output $1=$ gen_v1 .*imp_in;

output $2=$ gen_v2 .* imp_in;

out $1(i)=\operatorname{sum}($ output 1$) ;$

out2(i) $=\operatorname{sum}($ output2);

if $(i==1)$

seq_out $1=\operatorname{outl}(i)$;

else

seq_out2 = out2 (i);

seq out $1=[$ seq out 1 out 1 (i) $] ;$

end

seq_out $2=$ [seq_out2 out2 (i)];

end

clear $k$;

for $(k=1: 1: M)$ 


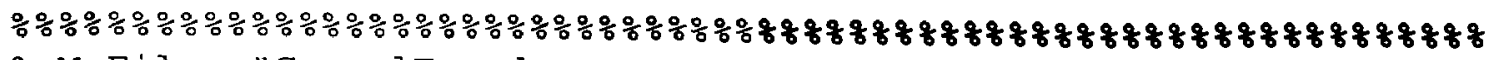

․ M-File: "ConvolEncoder.m"

\% AUTHOR: Boris Backovic

page: $2 / 3$

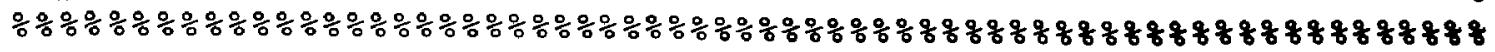

if $(k==1)$ else

imp_codeword $=[$ seq_out $1(\mathrm{k})$ seq_out2 $(\mathrm{k})]$; end

imp_codeword $=\left[i m p \_c o d e w o r d ~ s e q \_o u t 1(k)\right.$ seq_out2 $\left.(k)\right]$;

end

imp_codewords_len = length (imp_codeword);

data $=$ bit stream;

d_size = length (data);

cöded $=$ zeros $(1$, (imp_codewords_len + (num_output_per_bit * d_size

- num_output_per_bit)));

coded_length $=$ length $($ coded $) ;$

clear $t$;

for $(t=1: 1: d$ size)

parse $=\operatorname{data}(t)$;

if (parse $==1$ )

suma = imp_codeword;

else

suma $=$ zeros (imp_codewords_len);

end

suma $=\operatorname{suma}(I,:) ;$

clear $i$;

for $\left(i=(2 * t-1): 1:\left(i m p \_c o d e w o r d s \_l e n+(2 * t-1)-1\right)\right)$

if $((\operatorname{coded}(i)+\operatorname{suma}(i-(2 \star t-2)))==2)$

$\operatorname{coded}(i)=0$;

else

$\operatorname{coded}(i)=\operatorname{coded}(i)+\operatorname{suma}(i-(2 * t-2)) ;$

end

end

end

code_check = coded;

poly $\overline{1}=\operatorname{conv}($ data, gen_v1);

poly2 = conv (data, gen_v2);

polyl_len $=$ length $(\operatorname{dat} \bar{a})+$ length $($ gen_v1) -1 ;

poly2_len $=$ length $($ data $)+\operatorname{length}($ gen_v2 $)-1$;

clear $k$;

for ( $k=1: 1:$ poly1_len)

if $(\operatorname{rem}(\operatorname{pol} \bar{y}(k), 2) \sim=0)$

else

polyl $(k)=1$;

polyl $(k)=0$;

end 


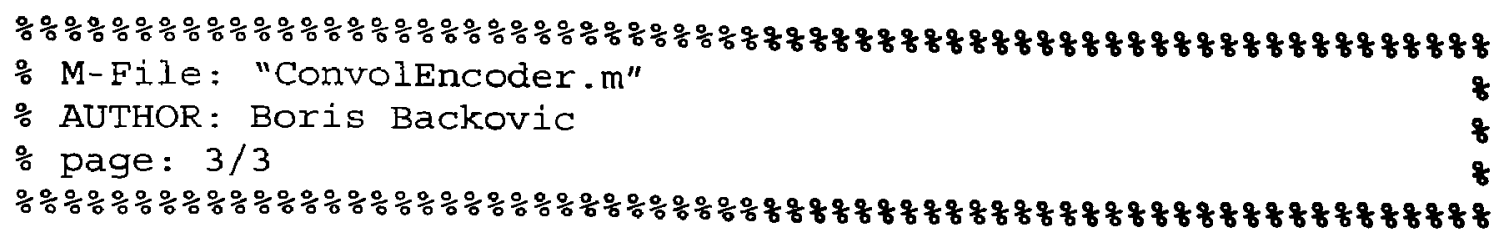

clear $k$;

for $(k=1: 1:$ poly 2 len $)$

if (rem (poly $2(k), 2) \quad \sim=0$ )

$\operatorname{poly}^{2}(\mathrm{k})=1$;

else

$\operatorname{poly}^{2}(k)=0 ;$

end

end

clear k;

for $(k=1: 1:$ polyl_len)

if $(\mathrm{k}==1)$

else

codeword $=\left[\right.$ poly $\left.1(k) p^{2} y^{2}(k)\right] ;$

codeword $=[$ codeword polyl $(k)$ poly2 $(k)]$;

end

d_gen_v1 = convert 2 dec $($ gen_v1);

o_gen_vl $=$ d2o (d_gen_vl);

d_gen_v2 = convert $2 \mathrm{de} c($ gen_v2);

o_gen_v2 $=$ d2o (d_gen_v2);

flush $=\operatorname{zeros}(1, M-1) ; \quad$ oflushing $M-1$ zeros for $M=4$ memory registers

message $=$ [data flush];

trel = poly2trellis $(\mathrm{M}$, [o_gen_v1 o_gen_v2]);

encoded_stream $=$ convenc (messäge, $\overline{\text { trel }})$;

if (sum (xor (coded, encoded_stream)) = 0)

display('WARNNING: Convolutional Encoding Performed with

Errors!!!! ' $\left.\backslash \mathrm{n}^{\prime}\right)$;

end

save ('Convol_Encoder.mat ', 'coded' , 'code_check', 'codeword' , trel', 'encoded_stream', 'data');

display ('OUT_ConvolEncoder.m') ; 


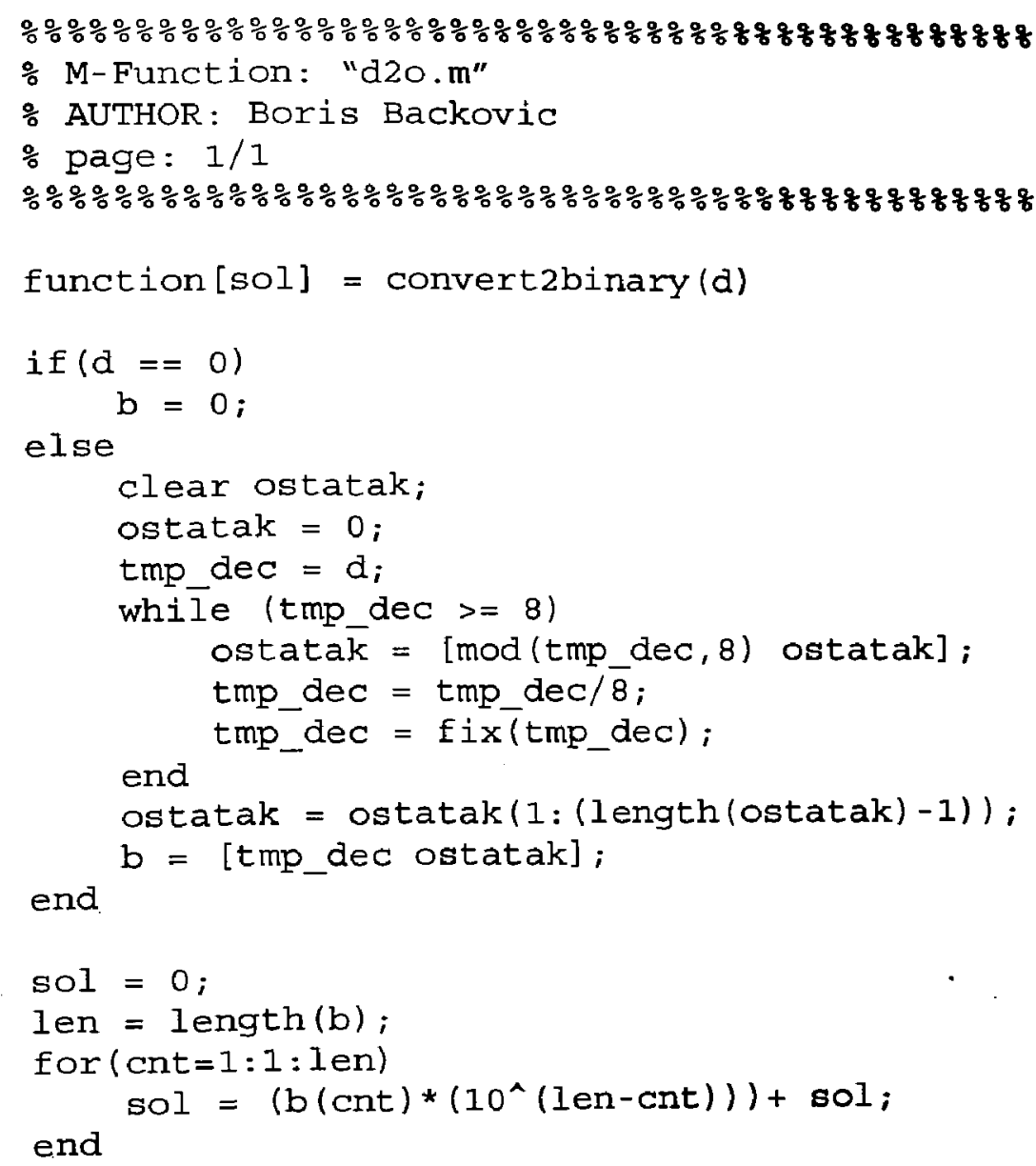




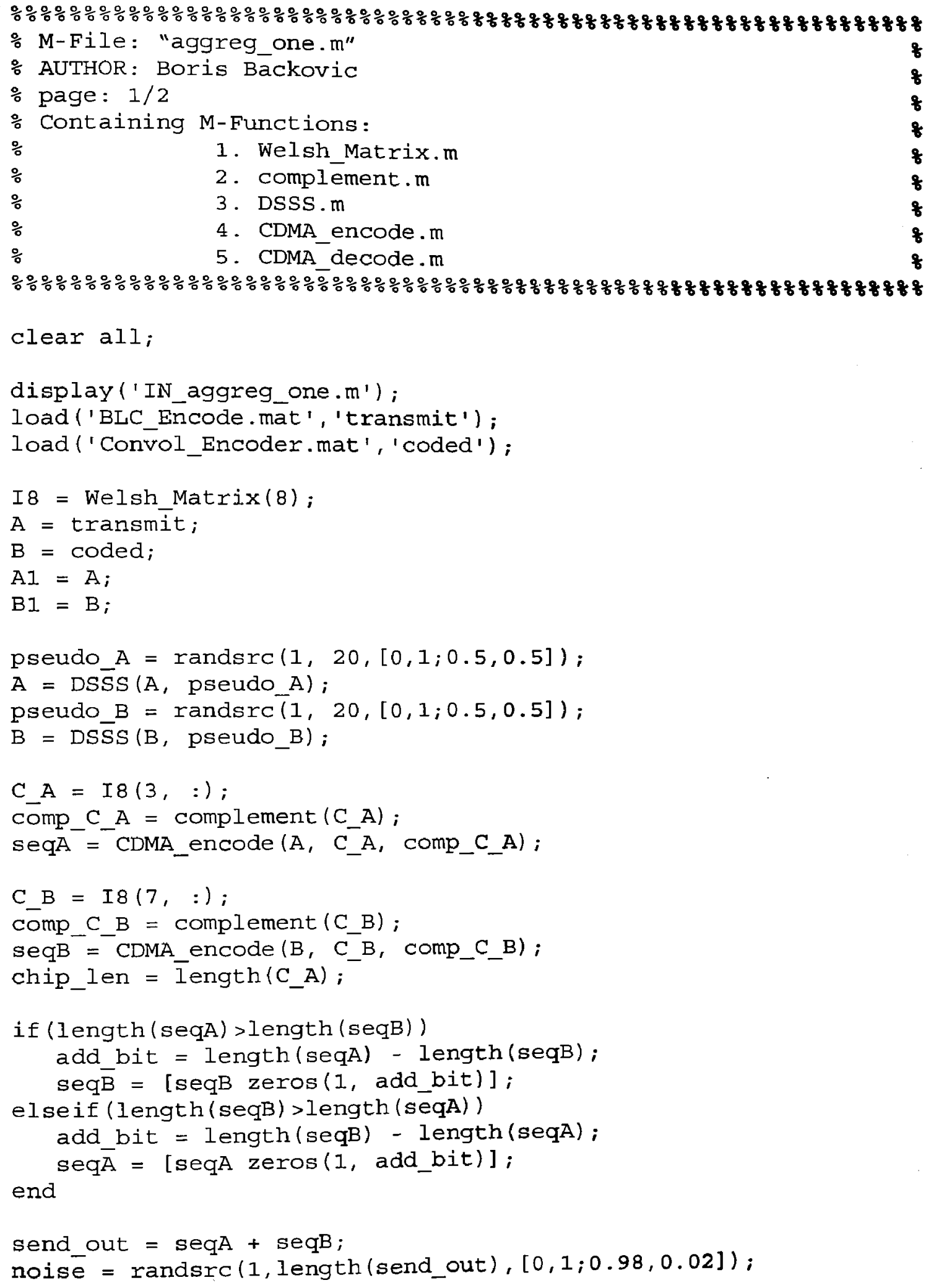




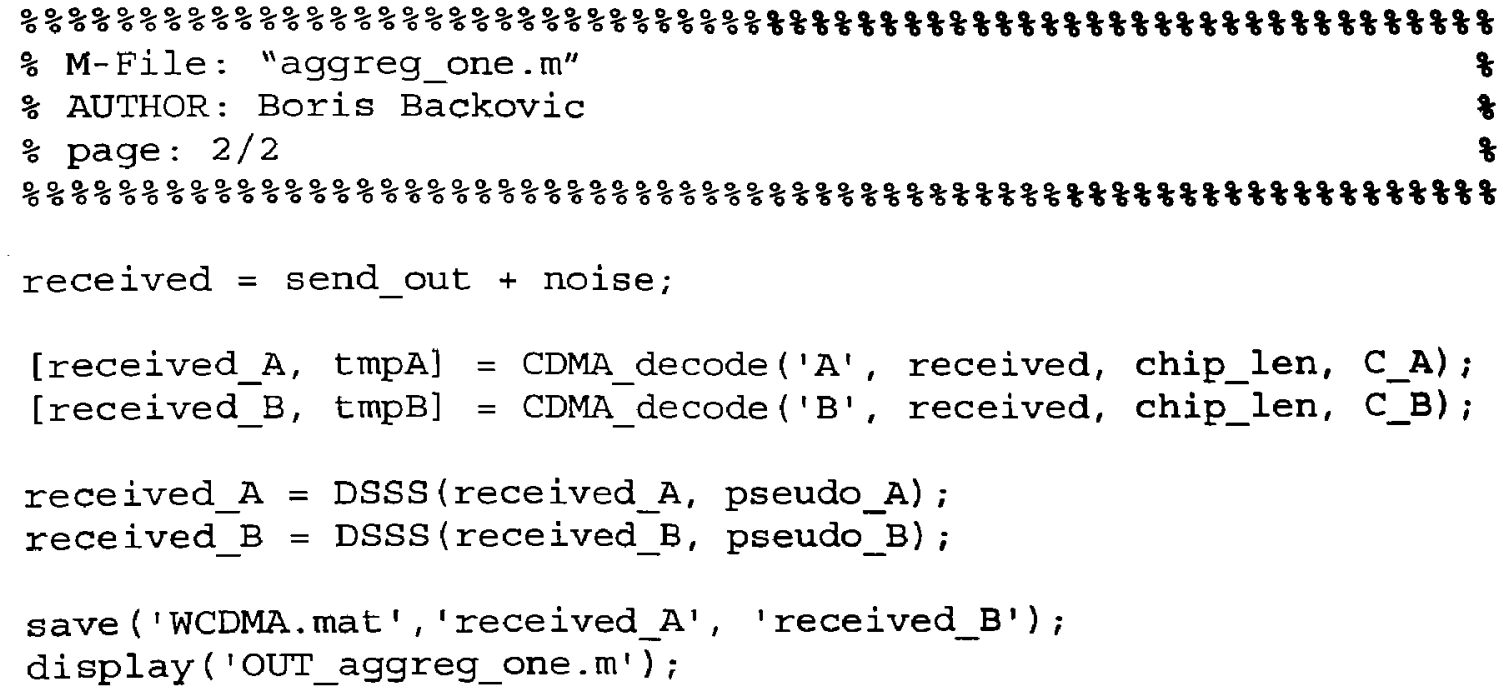




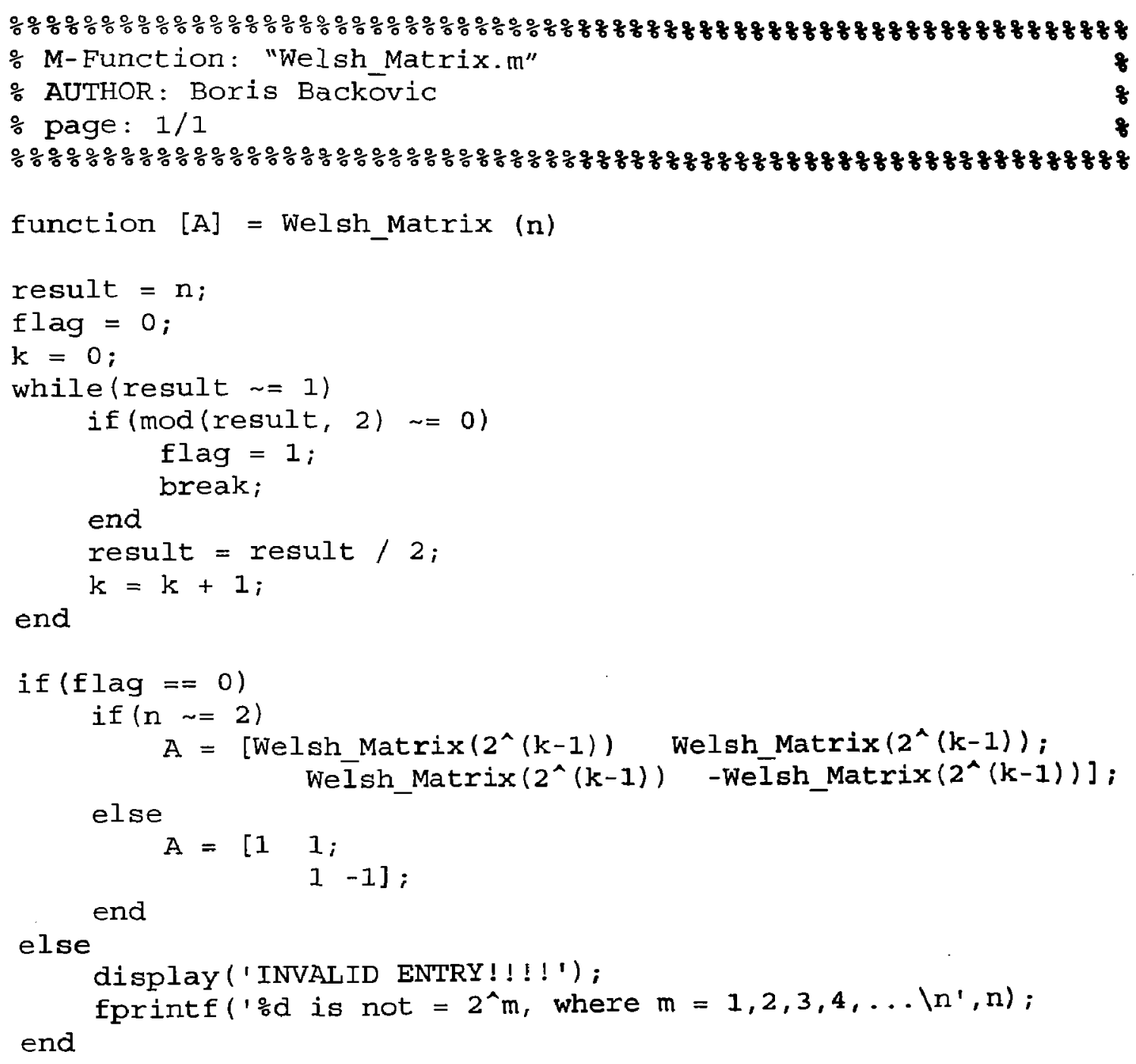




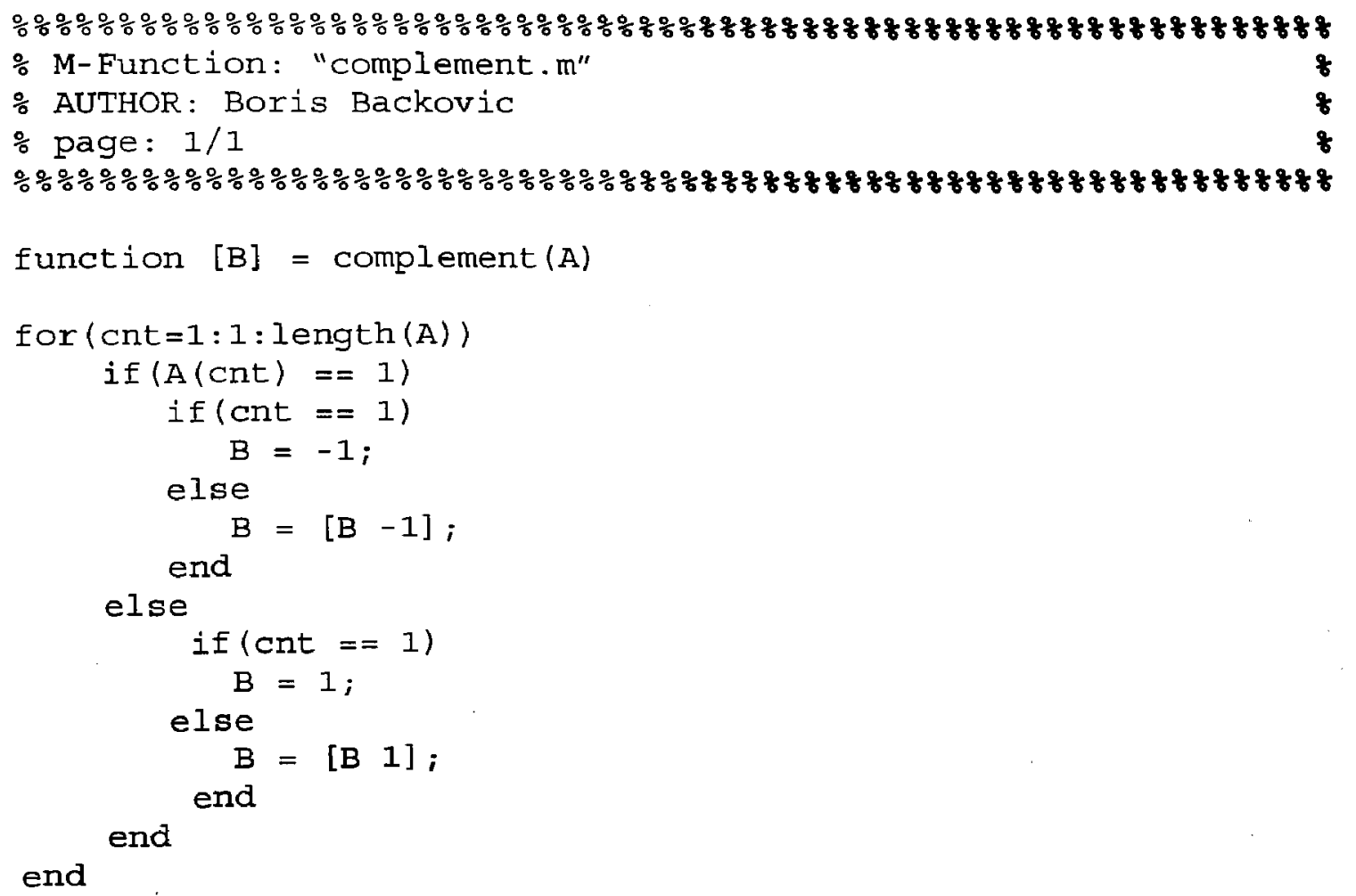




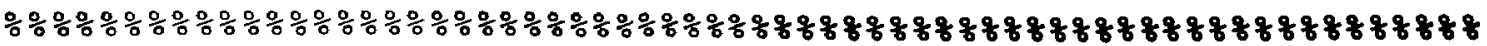

\% M-Function: "DSSS.m"

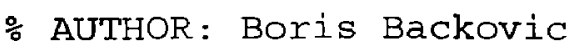

\% page: $1 / 1$

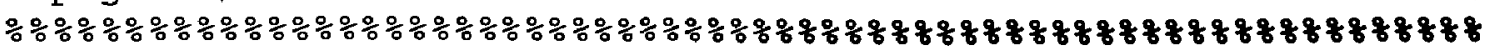

function [DS_A] $=$ DSSS ( $\mathrm{A}$, pseudo_A)

DS_A $=[0]$;

$\operatorname{cnt} 1=1$;

for (cnt $=1: 1:$ length $(A))$

$C=\operatorname{xor}(A(c n t)$, pseudo $A(c n t 1)) ;$

$\mathrm{DS} A=[\mathrm{DS} A \mathrm{C}]$;

$\operatorname{cnt} \bar{l}=\operatorname{cnt} \overline{1}+1$;

if (cnt $1>$ length (pseudo_A))

$\operatorname{cnt} 1=1$;

end

end

DS_A $=$ DS_A $(2$ : end $)$; 


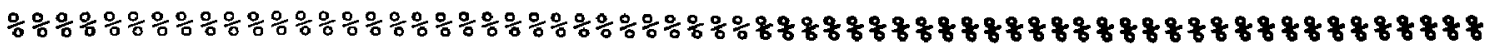

\% M-Function: "CDMA encode. $m$ "

\% AUTHOR: Boris Backovic

\% page: $1 / 1$

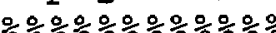

function $[$ seq $A]=$ CDMA_encode $(A, C$ C $A$, comp_C_A $)$

seqA $=[0] ;$

for ( $i=1: 1:$ length $(A)$ )

if $(A(i)==1)$

seq $A=\left[\operatorname{seg} A C_{-} A\right]$;

else

seqA $=[$ seq $A$ comp_C_A $] ;$

end

end

$\operatorname{seq} A=\operatorname{seq} A(2:$ end $) ;$ 


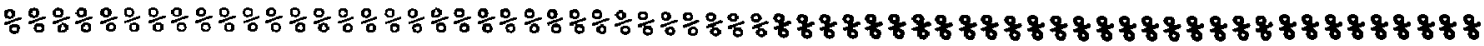

\% M-Function: "CDMA decode.m"

\% AUTHOR: Boris Backovic

\% page: $1 / 1$

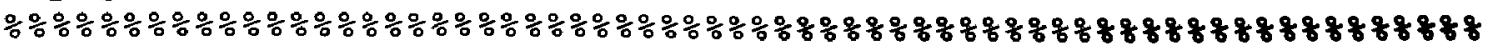

function [received_A, tmpA] = CDMA_decode (A, send, chip_len, C_A)

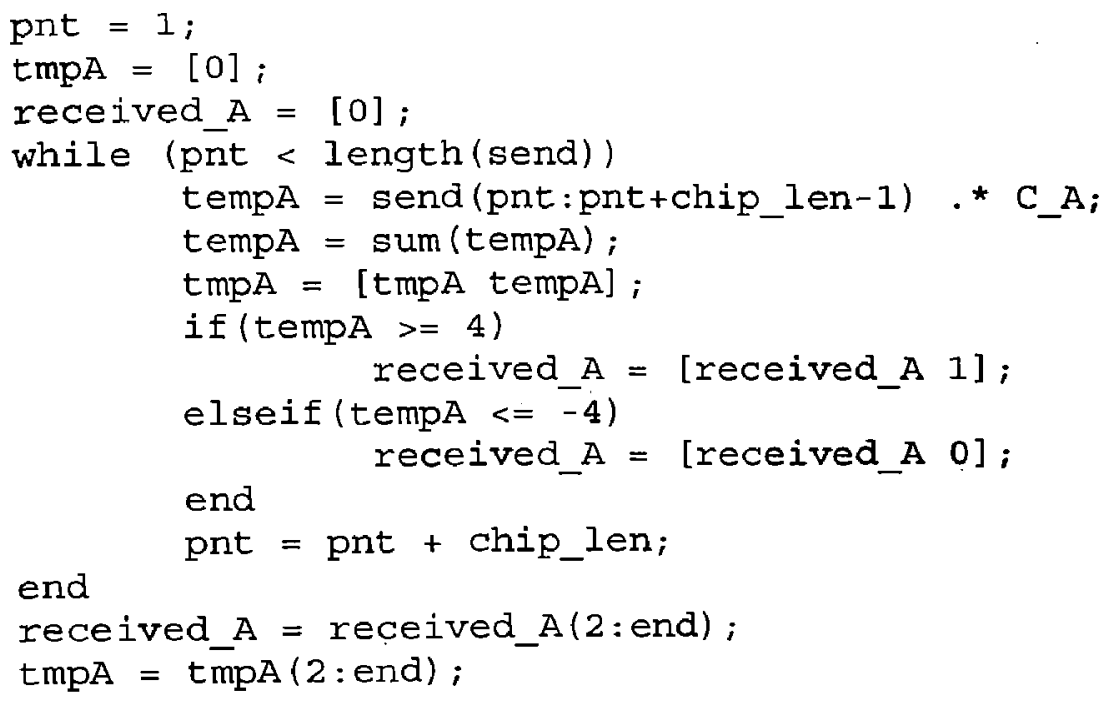




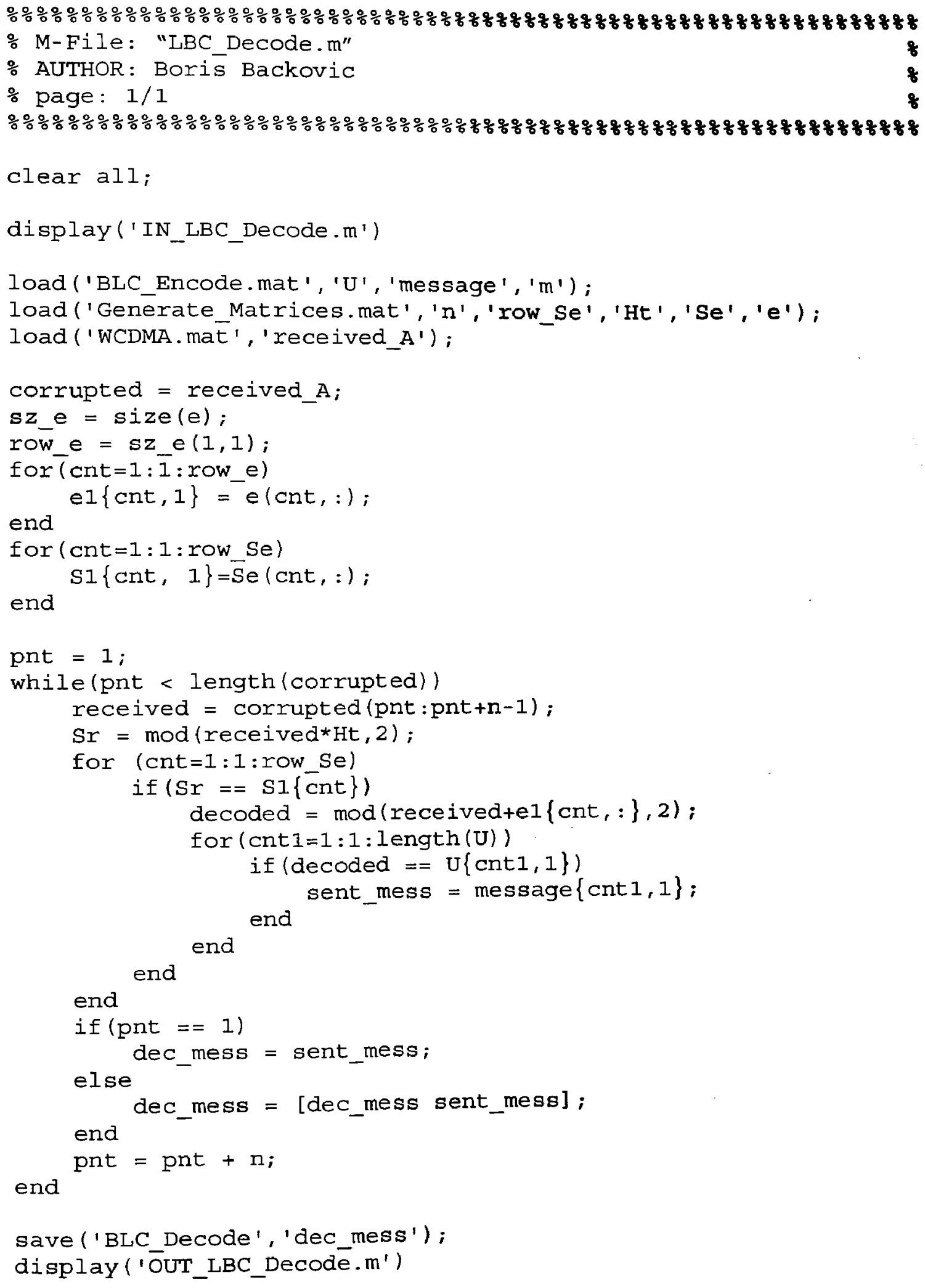




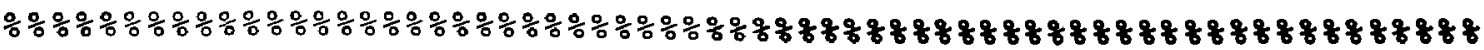

\% M-File: "Viterbi Decoder.m"

\% AUTHOR: Boris Backovic

\% page: $1 / 1$

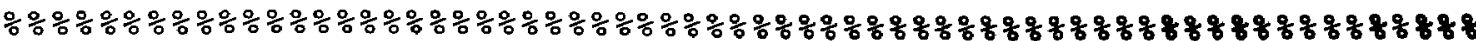

clear all;

display ('IN_Viterbi_Decoder.m') ;

load ('Convol Encoder.mat');

load ('WCDMA. mat', 'received_B');

coded $=\operatorname{cat}(2$, received_B, $\operatorname{zeros}(1,34))$;

decoded bits = vitdec (coded, trel, 20, 'cont', 'hard');

decoded_bits = decoded_bits (21:length(decoded_bits));

if (sum (xor (data, decoded bits)) $\sim=0$ )

display('WARNNING: Convolutional Encoding Performed with

Errors!! ! ' ') ;

end

fprinte ('\n');

save ('Viterbi_Decoder.mat', 'decoded_bits');

display ('ouT_Viterbi_Decoder.m') ; 


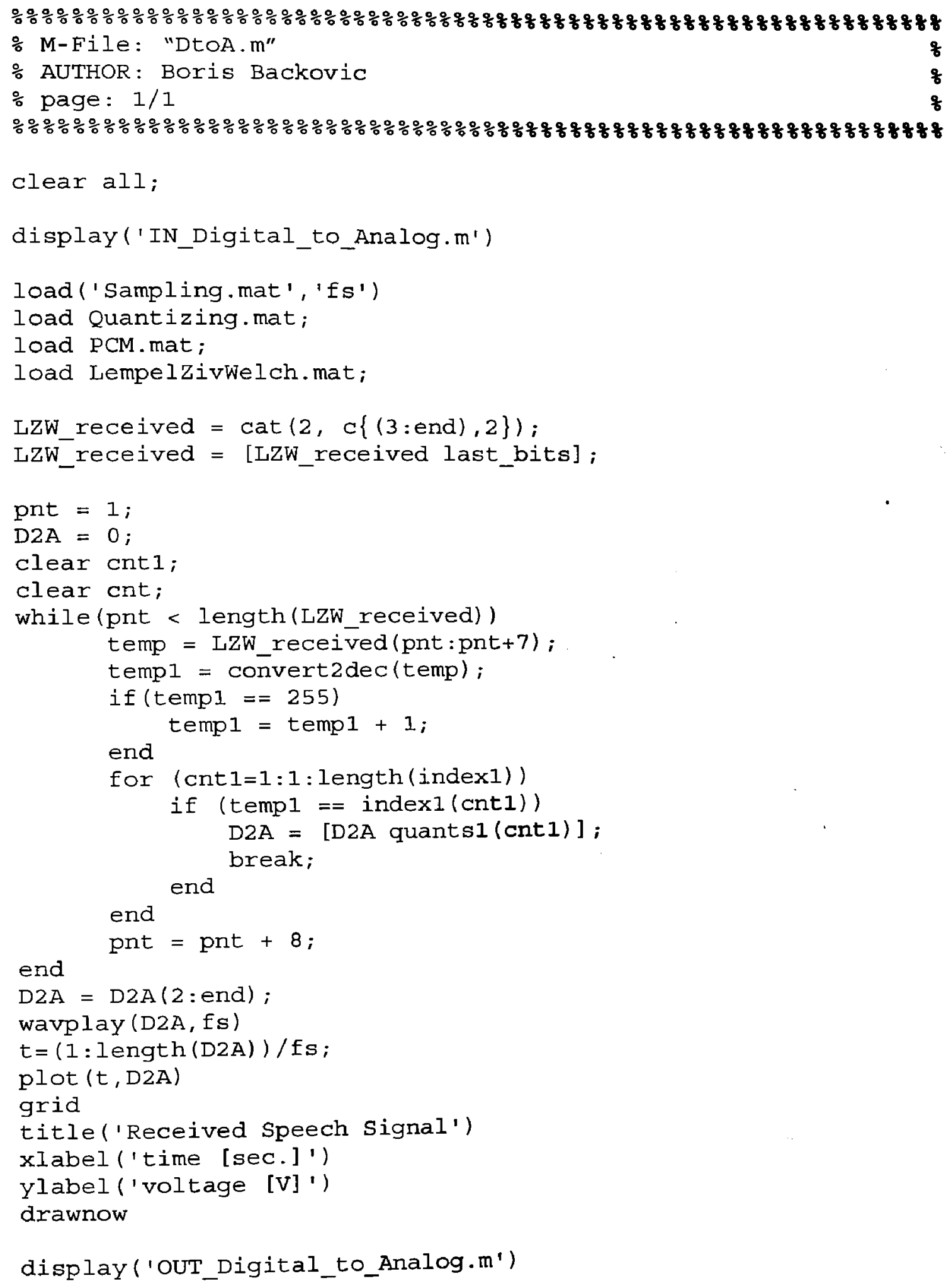




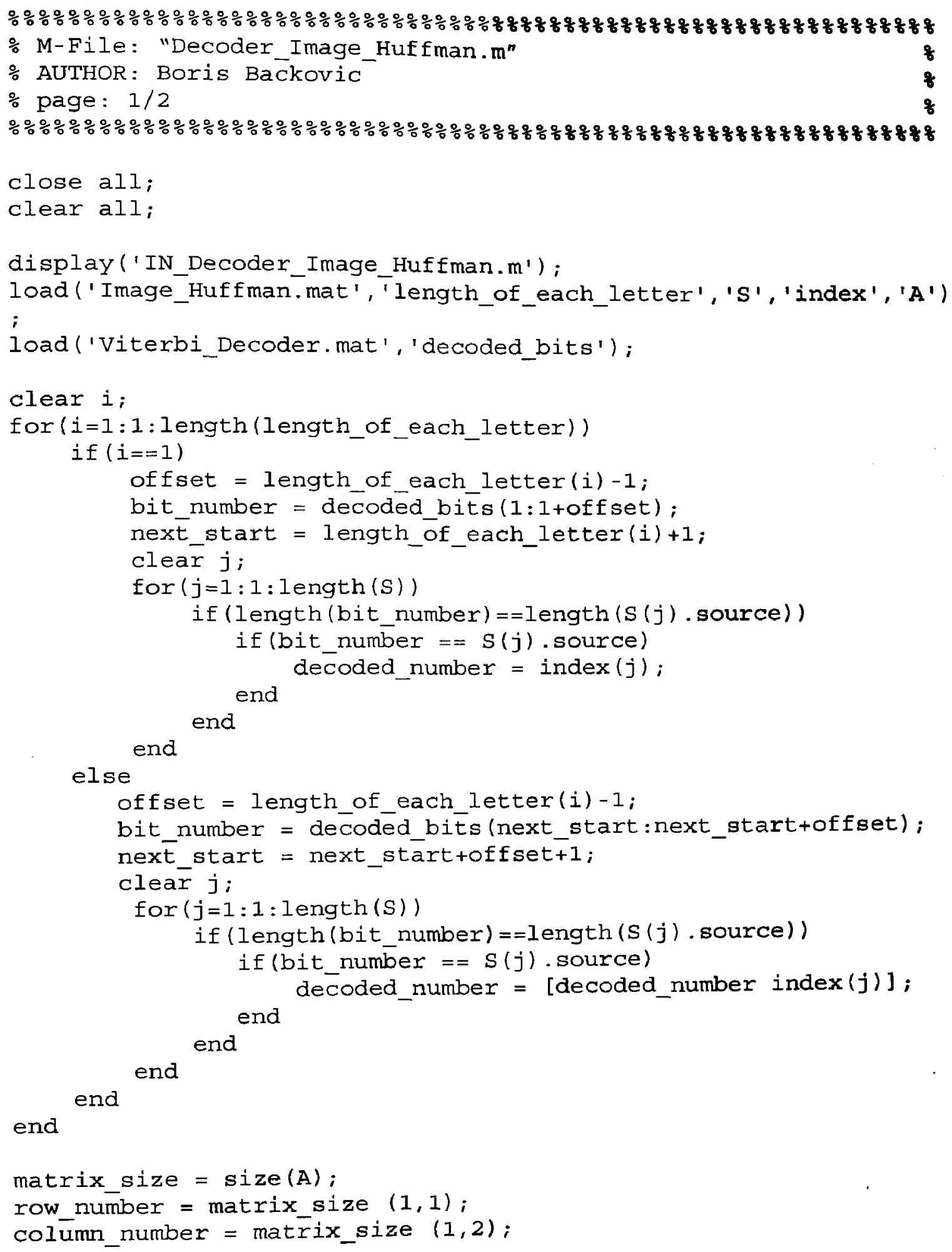




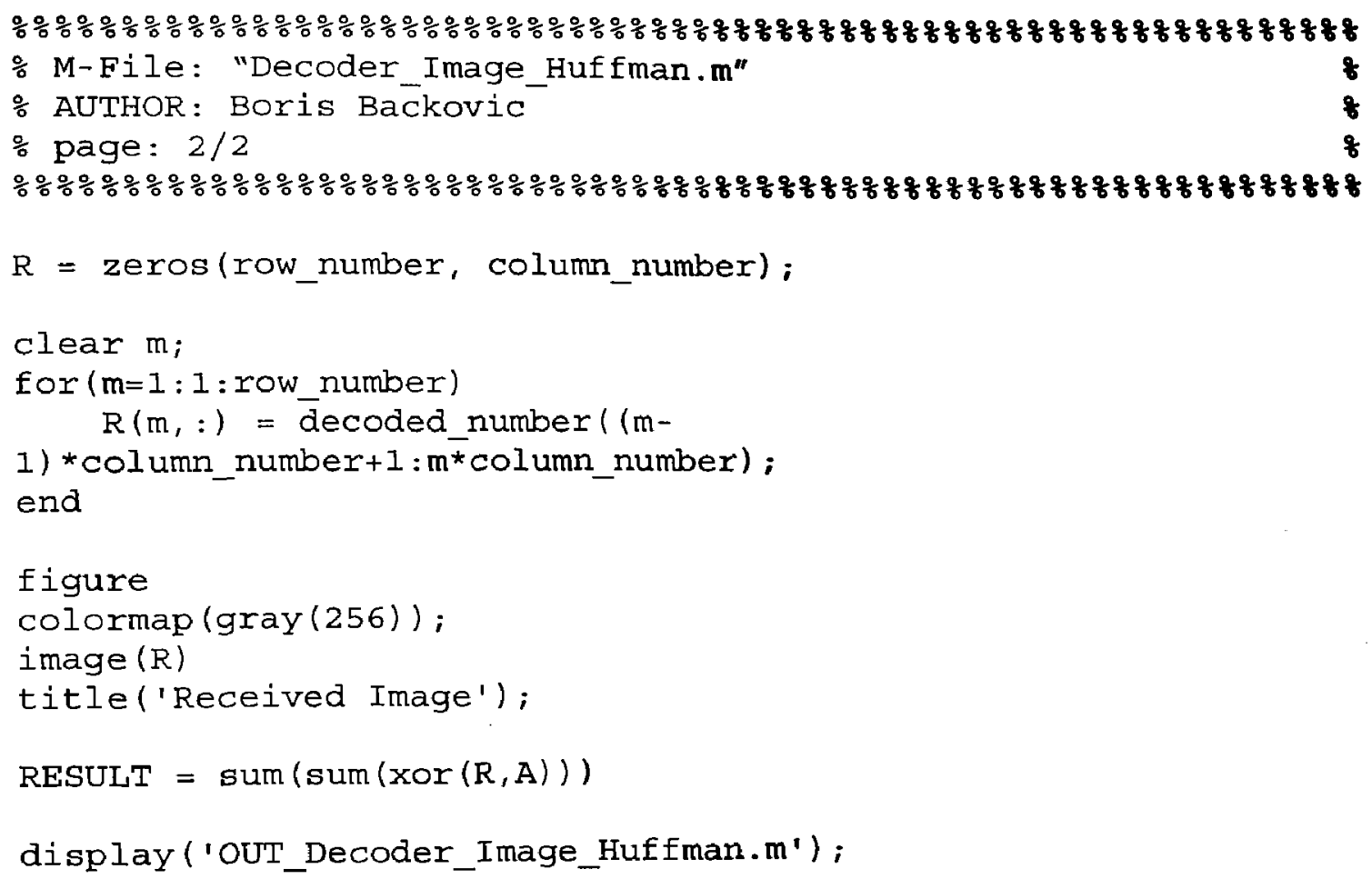


Appendix B:

Matlab Example Files 


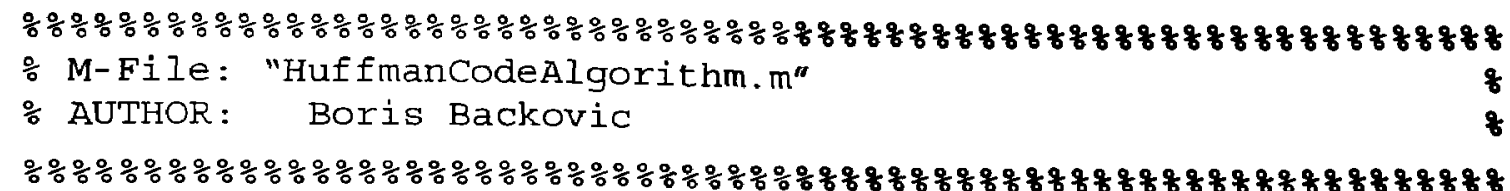

$\mathbf{s s}=\left[\begin{array}{llllllll}0.25 & 0.2 & 0.15 & 0.12 & 0.1 & 0.08 & 0.05 & 0.05\end{array}\right] ;$

$z=1$;

clear $i$;

for ( $i=1: 1:$ length (ss))

if ( $s(i) \sim=0$ )

$p(z)=s s(i) ;$

$\operatorname{index}(z)=i_{i}$

$z=z+1 ;$

end

end

$\mathrm{H}=0$;

clear $i$;

for $(i=1: 1:$ length $(p)$ )

$x=\log (p(i)) ;$

$\mathrm{x}=-\mathrm{x} / 0.69315$;

$y=p(i) * x ;$

$\mathrm{H}=\mathrm{H}+\mathrm{Y} ;$

end

symbols $=[1: 1:$ length $(\mathrm{p})]$;

$\mathrm{m}=\operatorname{length}(\mathrm{p})$;

code_dim = length $(p)$;

Huff = MakestructHuff (symbols,p);

sorted_Huff $=$ sorting_Huff (Huff, $m$, code_dim);

sorted_Huff.probability;

sorted_Huff.symbols;

while $(m>1)$

last_s = sorted_Huff.symbols $(:, m)$;

for ( $u=1: 1:$ length (last_s))

if (last_s $(u, 1) \sim 0$ )

for $(g=1: 1:$ code_dim)

if (Huff.codew $(g$, last_s $(u, 1)) \sim=0$ )

else 


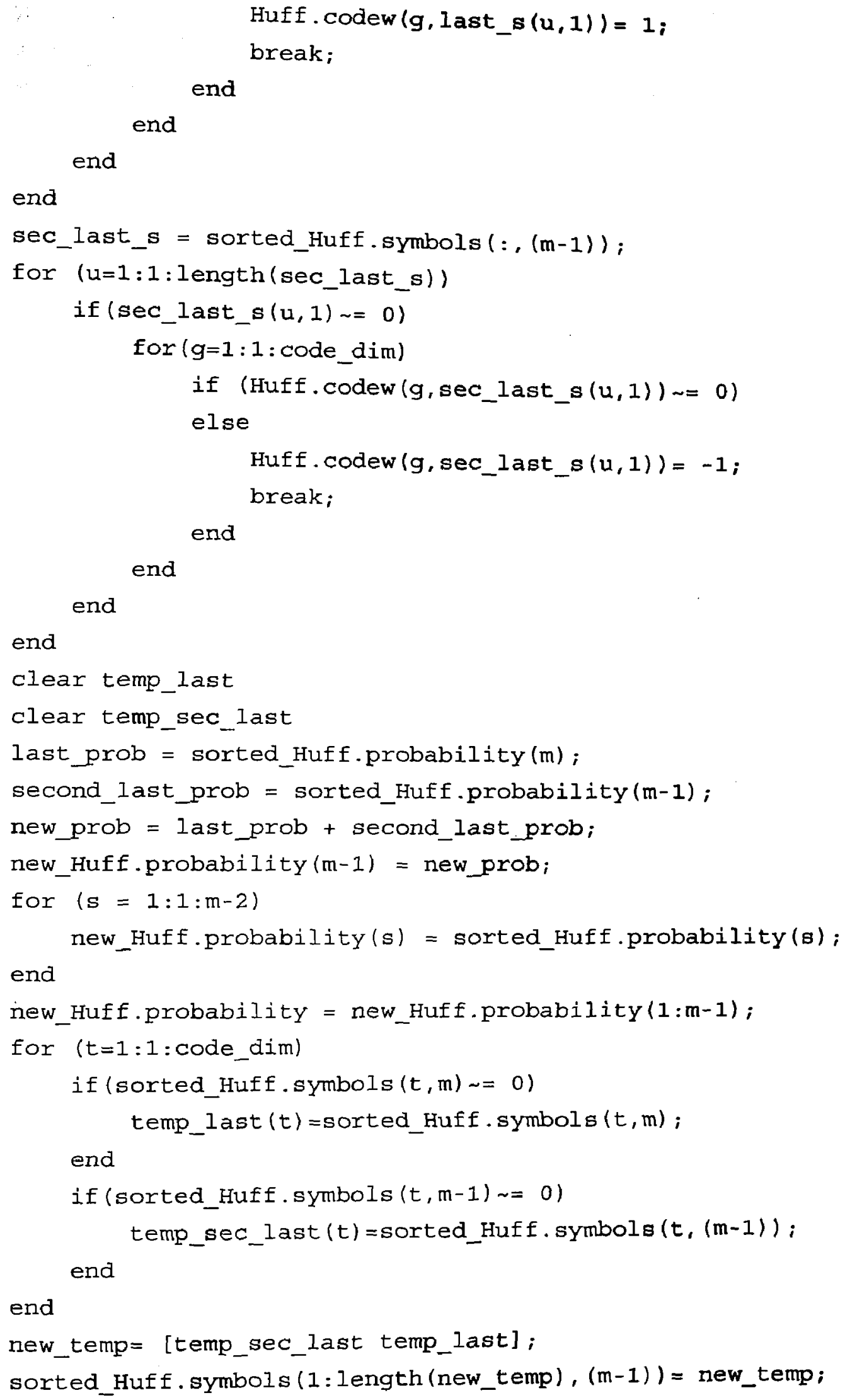




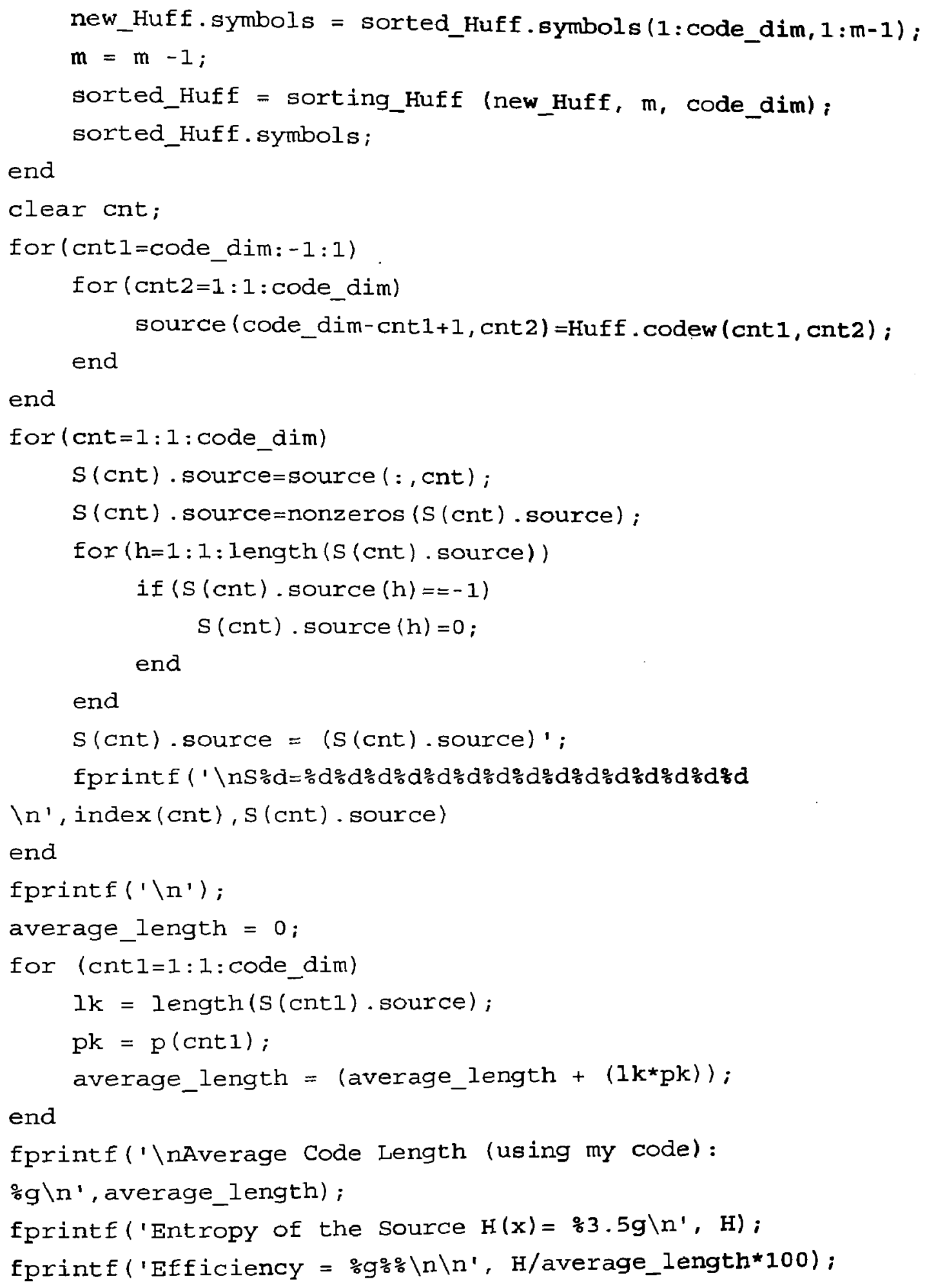


\% M-File: "LBC_Example.m"

응 AUTHOR: Boris Backovic,

$\mathrm{n}=5$;

$\mathrm{k}=3$;

$\mathrm{Ik}=$ eye $(\mathrm{k}) ;$

$\mathrm{P}=\left[\begin{array}{llll}0 & 1 ; 0 & 1 ; 1 & 1\end{array}\right] ;$

$G=[P[k]$;

Ink = eye $(n-k)$;

Ht $=\operatorname{cat}(1$, Ink, P);

$e=\left[\begin{array}{lllllllllllll}0 & 0 & 1 & 0 & 0 ; 1 & 0 & 0 & 0 & 0 ; 0 & 0 & 0 & 0 & 1\end{array}\right] ;$

sz_e $=\operatorname{size}(e) ;$

row_e $=s z$ sz $(1,1)$;

for (cnt $=1: 1:$ row_e)

$e I\{c n t, 1\}=e(c n t,:)$;

end

$s=\bmod \left(e^{*} H t, 2\right) ;$

sz_S $=\operatorname{size}(S)$;

row_s $=s z \_s(1,1)$;

column = sz_s $(1,2)$;

for (cnt $=1: 1:$ row_s $)$

$S 1\{\operatorname{cnt}, 1\}=S($ cnt,$:)$;

end

$\mathrm{m}=\left[\begin{array}{llllllllllllllllllllllll}0 & 0 & 0 & 0 & 0 & 1 & 0 & 1 & 0 & 0 & 1 & 1 & 1 & 0 & 0 & 1 & 0 & 1 & 1 & 1 & 0 & 1 & 1 & 1\end{array}\right] ;$

pnt $=1$;

ind $=1$;

while (pnt < length $(\mathrm{m}))$

message $\{$ ind $x, 1\}=m($ pnt $:$ pnt $+k-1) ;$

$U\{$ indx, 1$\}=\bmod (\operatorname{message}\{\operatorname{indx}, 1\} * G, 2)$;

pnt $=$ pnt $+k_{i}$

ind $=$ ind $x+1$;

end

corrupted $=\left[\begin{array}{lllllllllllllll}1 & 0 & 1 & 0 & 0 & 1 & 1 & 1 & 0 & 1 & 1 & 0 & 0 & 0 & 0\end{array}\right] ;$

pnt $=1$;

while (pnt < length (corrupted))

received $=$ corrupted $($ pnt $:$ pnt $+n-1) ;$ 


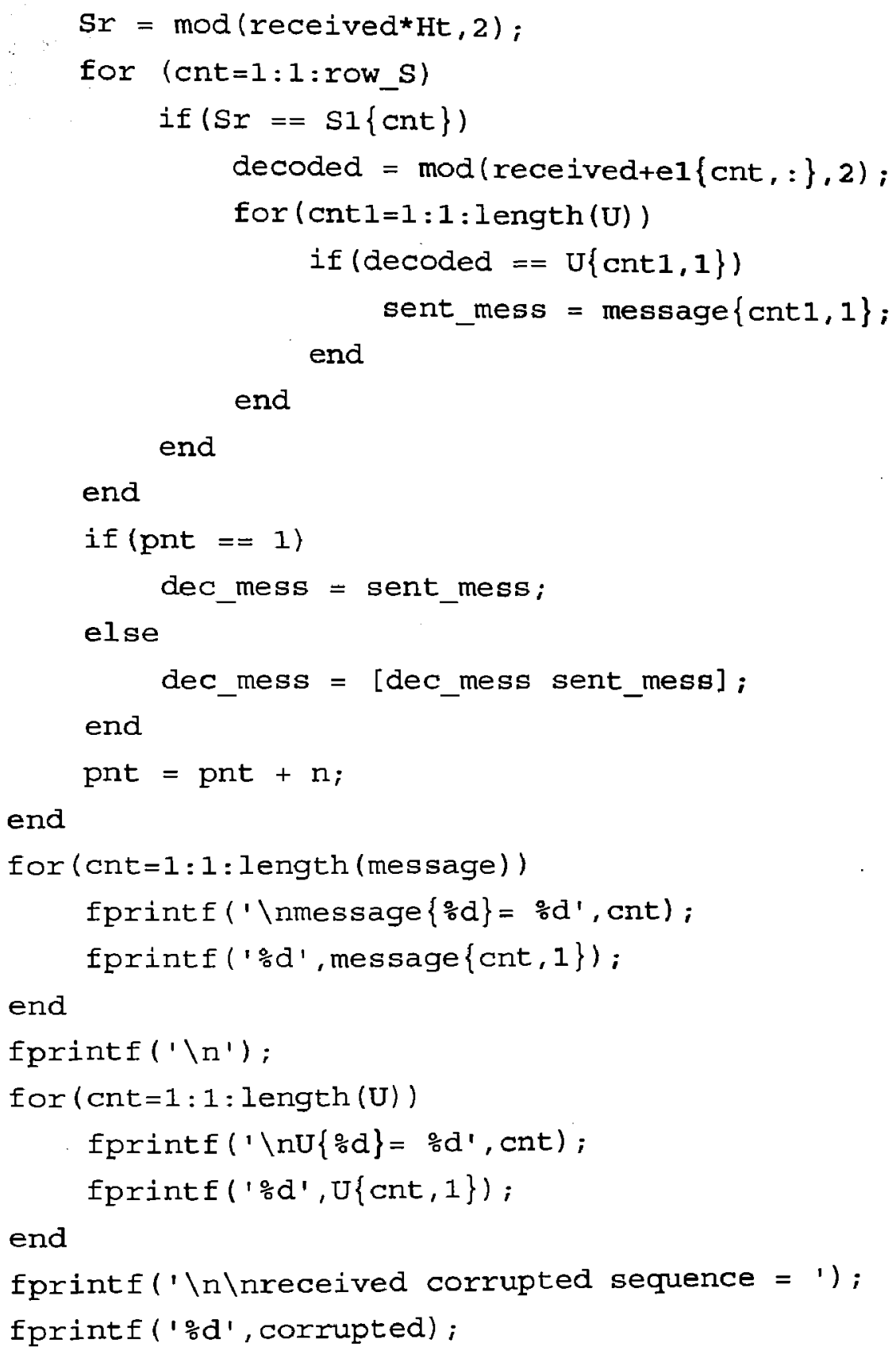




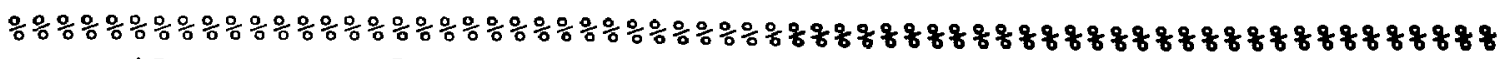

\% M-File: "ConvolExample.m"

․ AUTHOR: Boris Backovic,

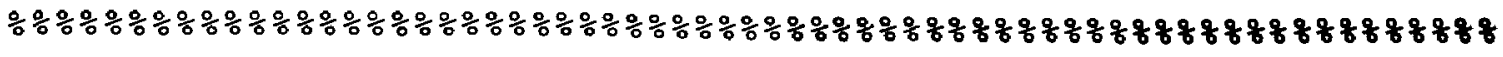

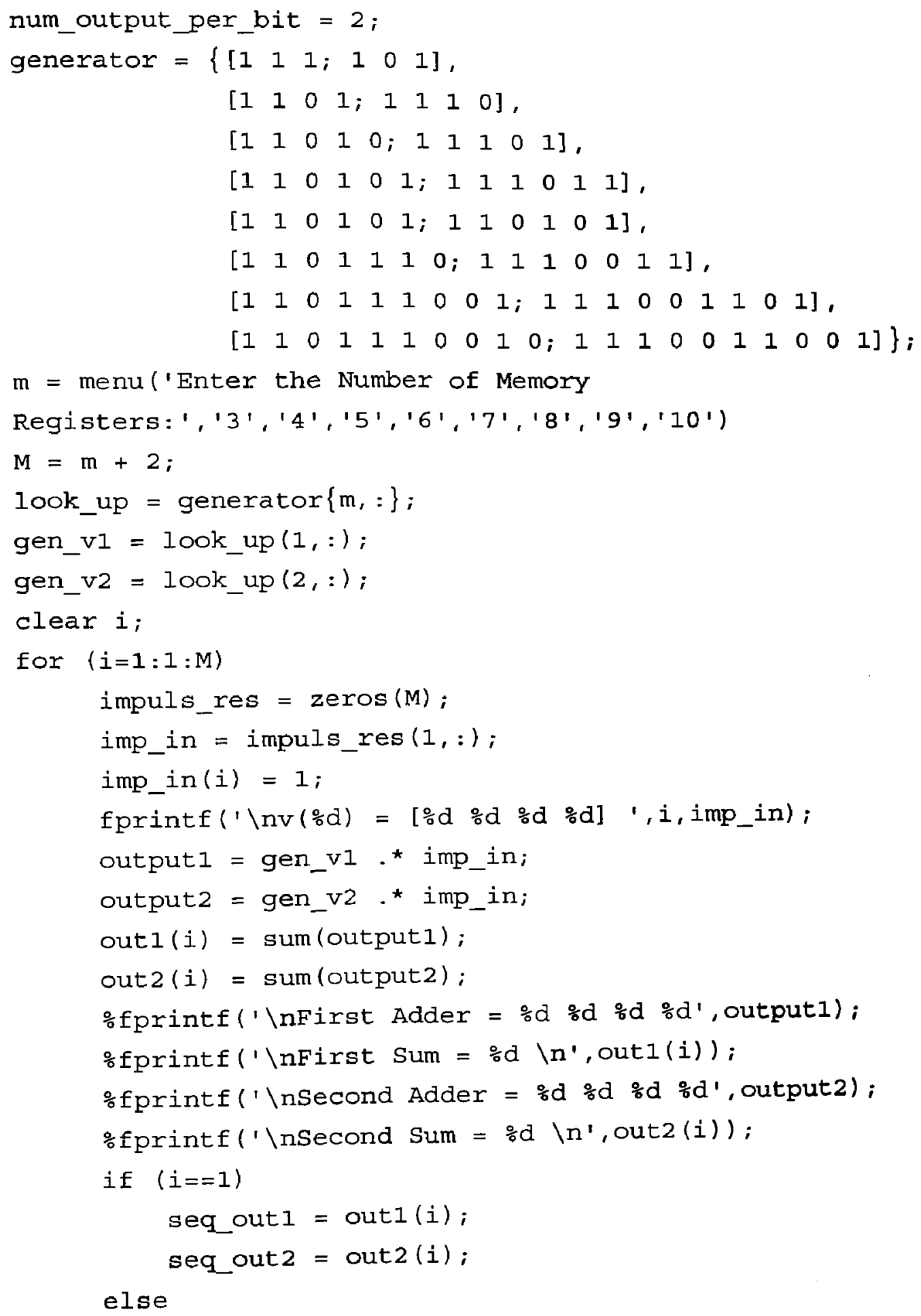




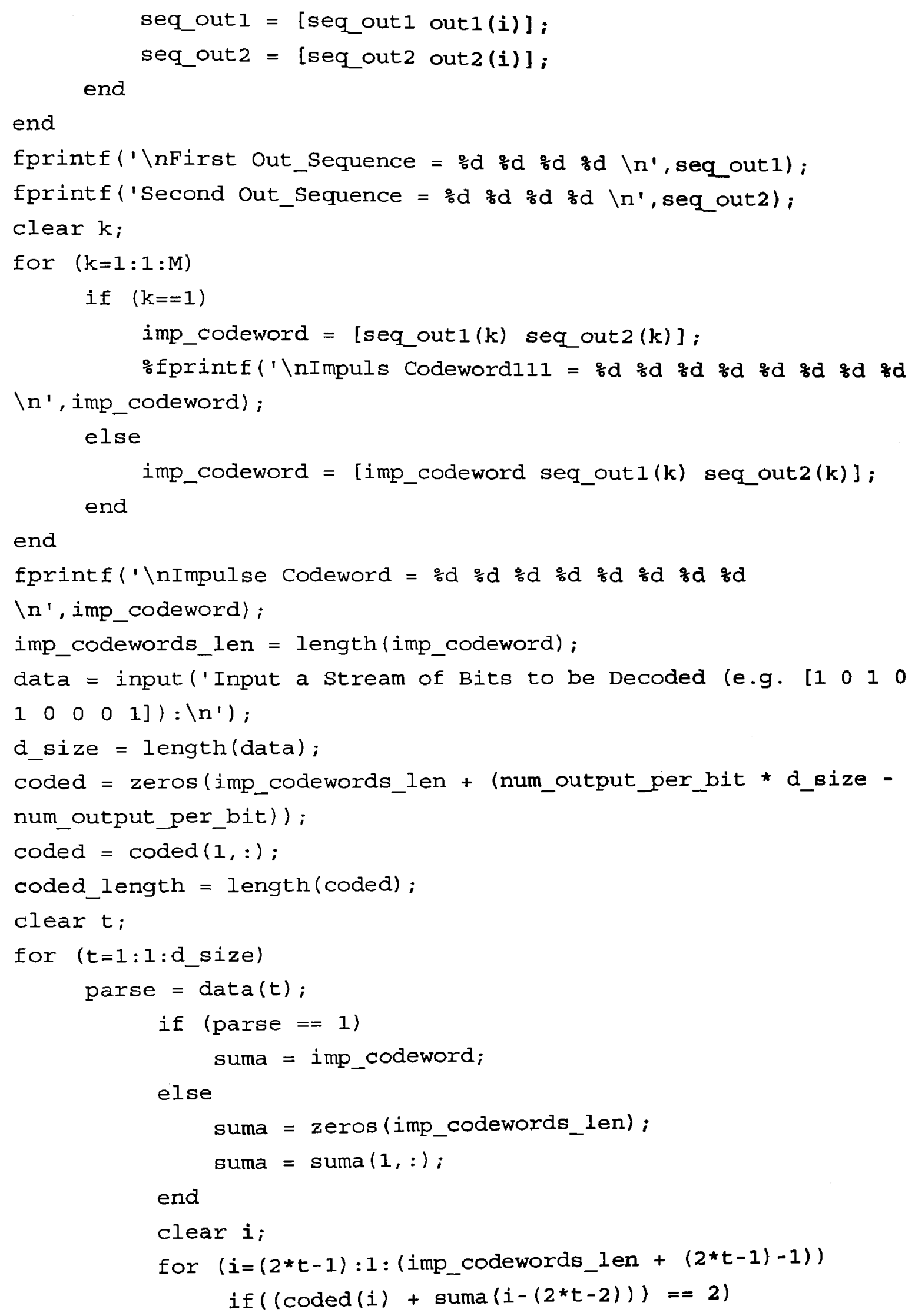




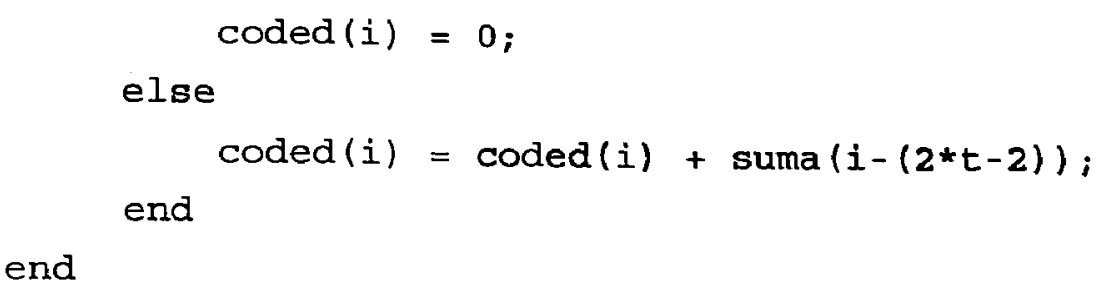

end

fprinte (' $\left.\backslash \mathrm{n}^{\prime}\right)$;

display('Connection Representation');

fprinte ('Coded: ');

fprinte ('\%d ', coded);

polyl = conv (data, gen_vl);

poly2 = conv (data, gen_v2);

poly1_len $=$ length $($ data $)+\operatorname{length}($ gen_v1 $)-1_{i}$

poly ${ }^{2}$ len $=$ length $($ data $)+\operatorname{length}($ gen_v2 $)-1 ;$

clear k;

for ( $k=1: 1:$ poly 1 len)

if (rem (polyl $(k), 2) \sim=0$ )

$\operatorname{poly} I(k)=1$;

else

$\operatorname{poly} 1(k)=0 ;$

end

end

clear k;

for ( $k=1: 1:$ poly2_len)

if (rem (poly2 $(\mathrm{k}), 2) \sim=0$ )

$\operatorname{poly}^{2}(\mathrm{k})=1$;

else

$$
\operatorname{poly}^{2}(\mathrm{k})=0 ;
$$

end

end

clear $\mathrm{k}$;

for $(k=1: 1:$ poly1_len)

if $(\mathrm{k}==1)$

codeword $=\left[\operatorname{poly} 1(k) \operatorname{pol}^{2}(k)\right]$;

else

codeword $=[$ codeword poly1 $(k)$ poly2 $(k)] ;$

end

end 


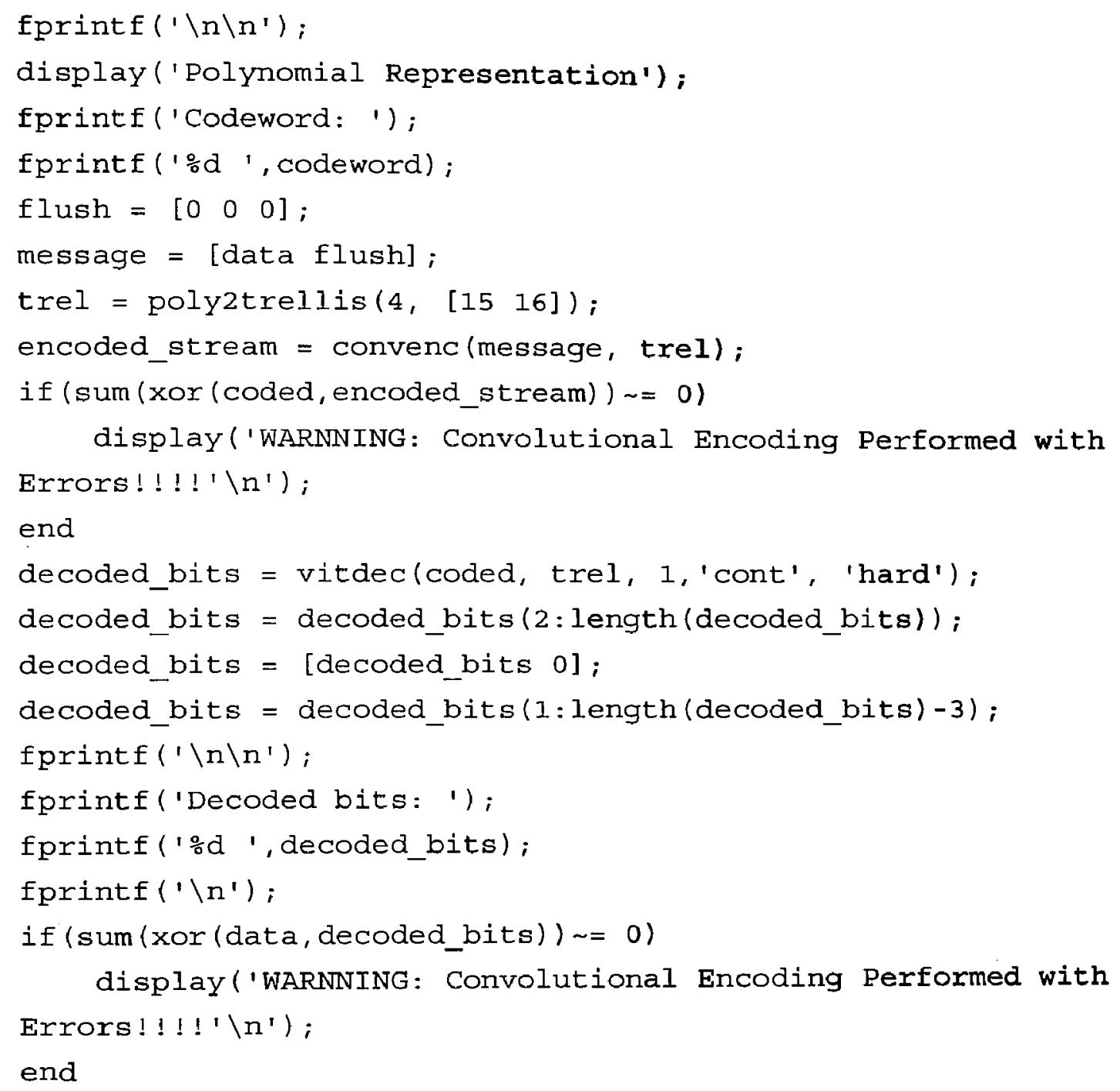

